\title{
Exorcising ghosts in induced gravity
}

\author{
Gaurav Narain ${ }^{\mathrm{a}}$ \\ Key Laboratory of Theoretical Physics, Kavli Institute for Theoretical Physics China (KITPC), Institute of Theoretical Physics (ITP), Chinese \\ Academy of Sciences (CAS), Beijing 100190, People's Republic of China
}

Received: 14 June 2017 / Accepted: 21 September 2017 / Published online: 16 October 2017

(C) The Author(s) 2017. This article is an open access publication

\begin{abstract}
Unitarity of the scale-invariant coupled theory of higher-derivative gravity and matter is investigated. A scalar field coupled with a Dirac fermion is taken as the matter sector. Following the idea of induced gravity the EinsteinHilbert term is generated via dynamical symmetry breaking of scale invariance. The renormalisation group flows are computed and one-loop RG improved effective potential of scalar is calculated. The scalar field develops a new minimum via the Coleman-Weinberg procedure inducing the Newton constant and masses in the matter sector. The spin- 2 problematic ghost and the spin- 0 mode of the metric fluctuation get a mass in the broken phase of the theory. The energy dependence of the vacuum expectation value in the RG improved scenario implies a running for the induced parameters. This sets up platform to ask whether it is possible to evade the spin-2 ghost by keeping its mass always above the running energy scale? In broken phase this question is satisfactorily answered for a large domain of coupling parameter space where the ghost is evaded. The spin- 0 mode can be made physically realisable or not depending upon the choice of the initial parameters. The induced Newton constant is seen to vanish in the ultraviolet case. By properly choosing parameters it is possible to make the matter fields physically unrealisable.
\end{abstract}

\section{Introduction}

Finding a well-defined and mathematically consistent theory of quantum gravity is one of the most important problems of theoretical physics. Moreover, finding experimental evidence validating or falsifying one is equally hard. Presently there are several models of quantum gravity which are aimed at studying the quantum nature of space-time and investigating physics at ultra-high energies. Recently a minimumlistic model in the framework of four dimension quantum field theory (QFT) in Lorentzian space-time was investigated, which

\footnotetext{
a e-mail: gaunarain@itp.ac.cn
}

was shown to be renormalisable to all loops [1,2], and which was recently shown to be unitary $[5,6]$ (see also the references therein). This then offers a sufficiently good and simple model of a quantum field theory of gravity whose arena can be used to investigate physics at ultra-high energies.

In this paper, motivated by the results of [2-8] we study the scale-invariant higher-derivative gravitational system coupled with matter fields. These constitute interesting systems. The scale-invariant purely gravitational sector consist of only dimensionless couplings. This makes the theory perturbatively renormalisable to all loops in four space-time dimensions by power-counting $[9,10]$ (for classical picture of these theories see $[1,11])$. Coupling this with scale-invariant matter sector does not change the picture. The resulting theory is still perturbatively UV renormalisable in four dimensions due to lack of any dimensionful parameter. Classically the matter sector, however, has local conformal invariance, which is broken under quantum corrections due to conformal anomalies (local and non-local) $[12,13]$. The interesting thing to note here is that in this quantum theory the counter terms generated still possess a scale-invariant structure (due to the lack of any dimensionful parameter in theory) [14]. This therefore preserves the renormalizability of the theory [2], even though trace anomalies are present.

Scale-invariant gravitational systems coupled with matter have been investigated in past. Some of the first studies were conducted in $[4,9,10,15,16]$, where the renormalisation group running of various couplings was computed and fixed point structure was analysed. Further investigation for more complicated systems were done in [17-22] (see also the book [23]). Matter coupling with conformal quantum gravity along with Gauss-Bonnet term were investigated in [24,25]. Recently it has gained some momentum and these models have been reinvestigated [26,30-33]. The purpose in these papers were to see if it is possible to generate a scale dynamically starting from scale-invariant system. In [26] the authors called their model 'Agravity'; the Planck scale is dynamically generated from the vacuum-expectation-value $(\mathrm{VeV})$ of the 
potential in an Einstein frame (not in a Jordan frame). By this one achieves a negligible cosmological constant, generates a Planck mass, and addresses the naturalness $[26,27]$ and inflation [28], but unitarity issues were not explored. ${ }^{1}$ In [30-33] the authors studies the issue of dynamical generation of scale via dimensional transmutation in the presence of background curvature. This also induces Einstein-Hilbert gravity and generates Newton's constant, but the unitarity problem was not addressed. An interesting idea has been suggested in $[34,35]$ by assuming an analogy with QCD, where the authors address the problems of ghost and tachyon using the wisdom acquired from the non-perturbative sector of QCD; it is argued that the gravitational theory enters a non-perturbative regime below the Planck scale.

The ideas of induced gravity goes back to long ago. It was first proposed in $[36,37]$, where the quantum matter fluctuations at high energy generate gravitational dynamics at low energy, inducing the cosmological and Newton gravitational constant. Another proposal suggested in [38-40] induces Einstein gravity spontaneously via symmetry breaking along the lines of the Higgs mechanism. Later in [41-45] the idea of generation of Einstein gravity via dynamical symmetry breaking was considered, following the methodology of Coleman-Weinberg [46]. In [45], metric fluctuations were also incorporated in the generation of the induced Newton constant. Around the same time induced gravity from Weyl theory was also studied [47-49]. Phase transitions leading to the generation of Einstein-Hilbert gravity due to loop effects from conformal factor coupled with scalar field were studied in [50]. In [51,52] the renormalisation group improved effective potential of the dilaton leads to running of the $\mathrm{VeV}$ inducing masses (along with Einstein-Hilbert gravity). Furthermore the authors make a proposal along the lines of $[3,4]$ to tackle ghost and tachyons. Cosmological consequences of these models were explored in $[53,54]$.

In this paper we therefore study scale-invariant gravitational and matter coupled systems in $(4-\epsilon)$ dimensional regularisation scheme. The beta functions are computed and compared with the past literature. The one-loop RG improved effective potential for the scalar field is computed by incorporating the quantum fluctuations of both matter and gravity [55]. The scale invariance is broken dynamically when the scalar field $\phi$ acquires a $\mathrm{VeV}$ via Coleman-Weinberg mechanism [46]. This in turn induces the gravitational Newton constant, the cosmological constant and masses in the matter sector. We work in Lorentzian signature and take the sign of $C_{\mu \nu \rho \sigma}^{2}$ (Weyl tensor square) to be negative, keeping the sign of $R^{2}$ term (where $R$ is Ricci scalar) to be always positive

\footnotetext{
1 In [29] a quantum mechanical treatment of four-derivative theories was suggested, which when suitably extended can tackle more complicated field theoretic systems. This can perhaps address issues of ghost and unitarity in a more robust manner.
}

(this is done to avoid tachyonic instabilities). These choice of signs further allows necessary convergence in Feynman $+i \epsilon$ prescription by suppressing fields modes with large action in the Lorentzian path integral. The sign of the $R \phi^{2}$ term is taken negative, so as to generate the right sign of Newton's constant and to avoid tachyonic instabilities in the broken phase. The negative sign of $C_{\mu \nu \rho \sigma}^{2}$ term is taken in order to satisfy the unitary criterion as stated in $[5,6]$. In this case we no longer have asymptotic freedom as has been observed in the Euclidean case in $[9,10,16,26,30-33]$. The VeV generated in RG improved effective potential has running, and therefore induces running in Newton's constant and the masses of matter fields. Due to the generation of the Einstein-Hilbert term in the action, the propagator of metric fluctuations in the broken phase gets modified, and the modes acquires mass. In this broken phase we investigate the problem of spin- 2 ghost by probing the running of its mass along the lines of $[5,6] .^{2}$

The outline of paper is following. In Sect. 2 the divergent part of the effective action is computed in $(4-\epsilon)$ dimensional regularisation scheme. The beta functions are obtained from it. In Sect. 3 one-loop renormalisation group improved effective potential for the scalar field is computed by incorporating quantum corrections from the gravitational and matter degrees of freedom. In Sect. 4 the breaking of scale invariance is studied via Coleman-Weinberg mechanism, which in turn induces the gravitational Newton constant and masses in the broken phase. RG equation for $\mathrm{VeV}$ is derived, which induces a running in the generated Newton constant and masses. In Sect. 5 a prescription to avoid spin- 2 massive ghost is given, where a procedure to pick the right set of initial conditions is stated. In Sect. 6 numerical analysis is done to give evidence showing that there exists a large domain of coupling parameter space where spin-2 massive ghost can be made physically unrealisable. Finally in Sect. 7 conclusions are presented.

\section{RG running}

In this section we compute the renormalisation group running of the various coupling parameters present in our scaleinvariant theory. We start first with the formalism and compute the various diagrams that contain UV divergences. These are then used to write the beta function of the various coupling parameters. We start by considering the path integral of the coupled system $(\hbar=c=1)$

\footnotetext{
2 These studies are conducted in the perturbative framework around the gaussian fixed point and is therefore different from studies done in the asymptotic safety scenario [56], where the RG running of couplings were computed using functional-renormalisation group in Euclidean signature [57-62], with spectral positivity $[63]$ and ghost $[64,65]$ issues analysed around a non-gaussian fixed point. Present work is also different from the recent studies done in the direction of finite and ghost free non-local quantum field theories of gravity [66-69], in the sense as it does not incorporates non-local features.
} 
$Z=\int \mathcal{D} \gamma_{\mu \nu} \mathcal{D} \phi \mathcal{D} \bar{\psi} \mathcal{D} \psi \exp \left[i\left(S_{\mathrm{GR}}+S_{\text {matter }}\right)\right]$,

where $S_{\mathrm{GR}}$ and $S_{\text {matter }}$ are given by

$$
\begin{aligned}
S_{\mathrm{GR}}= & \frac{1}{16 \pi} \int \mathrm{d}^{d} x \sqrt{-\gamma} \\
& \times\left[-\frac{1}{f^{2}}\left(R_{\mu \nu} R^{\mu \nu}-\frac{1}{3} R^{2}\right)+\frac{\omega}{6 f^{2}} R^{2}\right], \\
S_{\text {matter }}= & \int \mathrm{d}^{d} x \sqrt{-\gamma}\left[\frac{1}{2} \partial_{\mu} \phi \partial^{\mu} \phi-\frac{\lambda}{4} \phi^{4}\right. \\
& \left.\quad-\frac{\xi}{2} R \phi^{2}+\bar{\psi}\left(i \gamma^{\mu} D_{\mu}-y_{t} \phi\right) \psi\right],
\end{aligned}
$$

where the coupling parameters $f^{2}, \omega, \lambda, \xi$ and $y_{t}$ are all dimensionless, and the geometric quantities (curvature and covariant derivative) depends on metric $\gamma_{\mu \nu}$. In the fermionic part of action, the Dirac gamma matrices are defined via tetrads and inverse tetrads $\left(\gamma^{\mu}=e_{a}{ }^{\mu} \gamma^{a}, \gamma_{\mu}=e^{a}{ }_{\mu} \gamma_{a}\right)$, and $D_{\mu}$ is the spin-connection covariant derivative. We have

$D_{\mu}=\partial_{\mu}-\frac{i}{2} \sigma^{c d} \omega_{\mu c d}$.

Here Greek indices denote the space-time index, while the Latin indices denote the internal Lorentz index, and $\sigma_{c d}=$ $i / 4\left[\gamma_{c}, \gamma_{d}\right]$. The internal indices are raised and lowered using internal metric $\eta_{a b}$. For torsionless manifold the spin connection can be expressed in terms of the Christoffel connection $\Gamma_{\mu}^{\alpha}{ }_{\nu}$ (which can be re-expressed again in terms of tetrads) as

$$
\begin{aligned}
\omega_{\mu}{ }^{a d}=\frac{1}{2} & {\left[e^{a \rho}\left(\partial_{\mu} e^{d}{ }_{\rho}-\partial_{\rho} e^{d}{ }_{\mu}\right)-e^{d \rho}\left(\partial_{\mu} e^{a}{ }_{\rho}-\partial_{\rho} e^{a}{ }_{\mu}\right)\right.} \\
& \left.+e^{b}{ }_{\mu} e^{a \rho} e^{d \nu}\left(\partial_{\nu} e_{b \rho}-\partial_{\rho} e_{b \nu}\right)\right] .
\end{aligned}
$$

The dimensionless nature of coupling $f^{2}$ and $\omega / f^{2}$ results in a fully dimensionless scale-invariant coupled action.

We study the diffeomorphism invariant action of the coupled system using background field method [70,71] in $(4-\epsilon)$ dimensional regularisation scheme. It is advantageous, as by construction it preserves background gauge invariance. The field is decomposed into background and fluctuation. Keeping the background fixed the path integral is then reduced to an integral over the fluctuations. The gravitational metric field is decomposed into background and fluctuation, while the tetrads (and its inverse) are expressed in powers of this fluctuation field. The matter fields are similarly decomposed. The gauge invariance of the full metric field is then transformed into the invariance over the fluctuation field. To prevent over-counting of gauge-orbits in the path-integral measure, a constraint is applied on this fluctuation field, which results in appearance of auxiliary fields called ghosts. The effective action generated after integrating over the fluctuation and auxiliary fields still enjoys invariance over the background fields.
The quantum metric is written as

$\gamma_{\mu \nu}=\bar{g}_{\mu \nu}+h_{\mu \nu}$

where $\bar{g}_{\mu \nu}$ is some arbitrary (but fixed) background and $h_{\mu \nu}$ is the metric fluctuation. The full action can be expanded in powers of $h_{\mu \nu}$. The path-integral measure over $\gamma_{\mu \nu}$ is then replaced with measure over $h_{\mu \nu}$. Integrating over the fluctuation field implies that in some sense they will appear only as internal legs and never as external legs. The background gauge-invariant effective action formalism allows to choose a particular background for the ease of computation. In particular writing $\bar{g}_{\mu \nu}=\eta_{\mu \nu}+H_{\mu \nu}$ (while still keeping $H_{\mu \nu}$ generic) allows one to use the machinery of the flat spacetime QFT, thereby giving a particle interpretation to the internal $\left(h_{\mu \nu}\right)$ and external $\left(H_{\mu \nu}\right)$ legs, in the sense that the former behaves as virtual particle, while the latter is the corresponding external particle. In this manner one can compute the effective action for the external leg $H_{\mu \nu}$. Alternatively one can expand the full action around flat space-time directly, calling the fluctuation $h_{\mu \nu}^{\prime}$ though. This is a highly non-linear gauge theory with an infinite number of interactions terms (however, their couplings are related to each other by diffeomorphism invariance). Then following the usual strategy of background field method and writing $h_{\mu \nu}^{\prime}=H_{\mu \nu}+h_{\mu \nu}$, it is quickly seen that $H_{\mu \nu}$ is the external leg corresponding to the virtual particle given by $h_{\mu \nu}$. Integrating over quantum fluctuations $h_{\mu \nu}$ then gives the effective action in terms of $H_{\mu \nu}$ field.

One can then set up Feynman perturbation theory by expanding the original action in powers of $h_{\mu \nu}$ and $H_{\mu \nu}$. Similarly writing the scalar and fermion fields as

$\phi=\varphi+\chi, \quad \bar{\psi}=\bar{\theta}+\bar{\eta}, \quad \psi=\theta+\eta$,

one can expand the action in powers of fluctuations $\chi, \eta$ and $\bar{\eta}$. The piece which is quadratic in only fluctuations $\left(h_{\mu \nu}, \chi\right.$, $\eta$ and $\bar{\eta}$ ) gives the propagator while all the other terms gives the interactions vertices. In one-loop approximation the terms which are exclusively quadratic in fluctuations are retained, all other terms which involve higher powers of fluctuations ( $h_{\mu \nu}, \chi, \eta$ and $\bar{\eta}$ ) contribute in higher loops and will be ignored here. In fact for computing the running of all matter couplings (except $\xi$ ) it is sufficient to consider the situation with $H_{\mu \nu}=0$. However, in the case for computing running of $\xi$, terms up to (and at least) linear in $H_{\mu \nu}$ should be retained. Similarly if one is interested in studying behaviour or $R^{2}$ and $R_{\mu \nu} R^{\mu \nu}$ then one should at-least retain terms up to quadratic in $H_{\mu \nu}$.

\subsection{Gauge fixing and ghosts}

The path integration over the gravitational field is ill defined. This is a general feature of gauge theories where the gauge invariance (diffeomorphism invariance for gravity) relates 
two field configuration by gauge transformation. Such field configuration will contribute equally to the path integral. However, this will lead to over-counting. To prevent such over-counting, gauge invariance needs to be broken by constraining the gauge field. This procedure of systematically applying the constraint leads to ghost, which are elegantly taken care of by the Faddeev-Popov prescription [72].

However, in this style of breaking the invariance one may wonder whether the gauge (or diffeomorphism) invariance emerges in the effective action. To make sure that the effective action obtained after integrating out the fluctuation field is gauge invariant, background field method is followed. It is a method (and procedure) which guarantees that the effective action constructed using it will be background gauge invariant. Below we describe the procedure for gauge fixing in the background field method.

The diffeomorphism invariance of the full action in Eq. (2) implies that, for an arbitrary vector field $\epsilon^{\rho}$, the action should be invariant under the following transformation of the metric field variable:

$\delta_{D} \gamma_{\mu \nu}=\mathcal{L}_{\epsilon} \gamma_{\mu \nu}=\epsilon^{\rho} \partial_{\rho} \gamma_{\mu \nu}+\gamma_{\mu \rho} \partial_{\nu} \epsilon^{\rho}+\gamma_{\nu \rho} \partial_{\mu} \epsilon^{\rho}$,

where $\mathcal{L}_{\epsilon} \gamma_{\mu \nu}$ is the Lie derivative of the quantum metric $\gamma_{\mu \nu}$ along the vector field $\epsilon^{\rho}$. Decomposing the quantum metric $\gamma_{\mu \nu}$ into background $\left(\bar{g}_{\mu \nu}\right)$ and fluctuation $\left(h_{\mu \nu}\right)$ allows one to figure out the transformation of the fluctuation field, while keeping the background fixed. This will imply the following transformation of $h_{\mu \nu}$ :

$\delta_{D} h_{\mu \nu}=\bar{\nabla}_{\mu} \epsilon_{v}+\bar{\nabla}_{\nu} \epsilon_{\mu}+\epsilon^{\rho} \bar{\nabla}_{\rho} h_{\mu \nu}+h_{\mu \rho} \bar{\nabla}_{\nu} \epsilon^{\rho}+h_{\nu \rho} \bar{\nabla}_{\mu} \epsilon^{\rho}$,

where $\bar{\nabla}$ is the covariant derivative whose connection is constructed using the background metric. This is the full transformation of the metric fluctuation field. Ignoring terms which are linear in $h_{\mu \nu}$ allows one to investigate only the one-loop effects. These ignored terms are, however, mandatory when dealing with higher-loop effects. The invariance of the action is broken by choosing an appropriate gauge-fixing condition implemented via the Faddeev-Popov procedure.

The gauge-fixing action chosen for fixing the invariance under the transformation of the metric fluctuation field is given by

$$
\begin{aligned}
S_{G F}= & \frac{1}{32 \pi \alpha} \int \mathrm{d}^{d} x \sqrt{-\bar{g}}\left(\bar{\nabla}^{\rho} h_{\rho \mu}-\frac{1+\rho}{d} \bar{\nabla}_{\mu} h\right) Y^{\mu \nu} \\
& \times\left(\bar{\nabla}^{\sigma} h_{\sigma \nu}-\frac{1+\rho}{d} \bar{\nabla}_{\nu} h\right),
\end{aligned}
$$

where $\alpha$ and $\rho$ are gauge parameters, while $Y_{\mu \nu}$ is either a constant or a differential operator depending upon the gravitational theory under consideration. For the theory considered here in Eq. (2), we consider higher-derivative type gauge fixing with $Y_{\mu \nu}=\left(-\bar{g}_{\mu \nu} \bar{\square}+\beta \bar{\nabla}_{\mu} \bar{\nabla}_{\nu}\right)$, where $\bar{\square}=\bar{\nabla}_{\mu} \bar{\nabla}^{\mu}$.
Choosing $\rho=-1$ and $\beta=0$ correspond to Landau gauge condition. Taking $\alpha \rightarrow 0$ imposes the gauge condition sharply.

The ghost action is obtained following the Faddeev-Popov procedure [72]. In general if the gauge-fixing condition on the gravitational field $h_{\mu \nu}$ is written as $F_{\mu}=0$ (which here is $F_{\mu}=\bar{\nabla}^{\rho} h_{\rho \mu}-\frac{1+\rho}{d} \bar{\nabla}_{\mu} h$ ), we introduce it in the path integral by multiplying the latter with unity in the following form:

$$
1=\int \mathcal{D} F_{\mu}^{\epsilon}(\operatorname{det} Y)^{\frac{1}{2}} \exp \left[\frac{i}{32 \pi \alpha} \int \mathrm{d}^{d} x \sqrt{-\bar{g}} F_{\mu}^{\epsilon} Y^{\mu \nu} F_{v}^{\epsilon}\right] \text {, }
$$

where $F_{\mu}^{\epsilon}$ is the gauge transformed $F_{\mu}$. As $Y^{\mu \nu}$ contains a derivative operator, its determinant is non-trivial. The original path integral (without gauge fixing) being invariant under transformation Eq. (8) of the field $h_{\mu \nu}$ implies that a change of integration variable from $h_{\mu \nu}$ to $h_{\mu \nu}^{\epsilon}$ does not give rise to any jacobian in the path-integral measure. However, replacing the measure over $F_{\mu}^{\epsilon}$ with measure over $\epsilon^{\rho}$ introduces a non-trivial jacobian in the path integral. This is obtained as follows:

$\mathrm{d} F_{\mu}^{\epsilon}=\frac{\partial F_{\mu}}{\partial \epsilon^{\rho}} \mathrm{d} \epsilon^{\rho} \Rightarrow \mathcal{D} F_{\mu}^{\epsilon}=\operatorname{det}\left(\frac{\partial F_{\mu}}{\partial \epsilon^{\rho}}\right) \mathcal{D} \epsilon^{\rho}$.

In the background field formalism this jacobian consists of a background covariant derivative, background and fluctuation fields, and is independent of the transformation parameter $\epsilon^{\rho}$. This implies that it can be taken out of the functional integral over $\epsilon^{\rho}$. Changing the integration variable from $h_{\mu \nu}^{\epsilon}$ to $h_{\mu \nu}$, and ignoring the infinite constant generated by integrating over $\epsilon^{\rho}$, gives us the gauge-fixed path integral including the determinant.

The functional determinant appearing in Eq. (11) can be exponentiated by making use of appropriate auxiliary fields. Writing the functional determinant $(\operatorname{det} Y)^{1 / 2}$ as $(\operatorname{det} Y) \times$ $(\operatorname{det} Y)^{-1 / 2}$, allows to combine the former with the FaddeevPopov determinant in Eq. (11), which is then exponentiated by making use of anti-commuting auxiliary fields, while the latter $(\operatorname{det} Y)^{-1 / 2}$ is exponentiated by making use of commuting auxiliary fields. The former auxiliary fields are known as Faddeev-Popov ghosts, while those in the latter case are known as Nielsen-Kallosh ghosts [73,74]. The path integral of the full ghost sector is given by

$$
\begin{aligned}
& \int \mathcal{D} \bar{C}_{\mu} \mathcal{D} C_{\nu} \mathcal{D} \theta_{\alpha} \exp \left[-i \int \mathrm{d}^{d} x \sqrt{-\bar{g}}\right. \\
& \left.\times\left\{\bar{C}_{\mu}\left(Y^{\mu \nu} \frac{\partial F_{\nu}}{\partial \epsilon^{\rho}}\right) C^{\rho}+\frac{1}{2} \theta_{\alpha} Y^{\alpha \beta} \theta_{\beta}\right\}\right],
\end{aligned}
$$

where $\bar{C}_{\mu}$ and $C_{v}$ are Faddeev-Popov ghost fields arising from the gauge fixing in the gravitational sector, and $\theta_{\mu}$ is the commuting ghost arising due to fact that $Y_{\mu \nu}$ contains derivatives. 
In the case when $F_{\mu}$ is given as in Eq. (9), the FaddeevPopov ghost action is given by

$S_{\mathrm{gh}}^{F P}=-\int \mathrm{d}^{d} x \sqrt{-\bar{g}} \bar{C}_{\mu} X_{\rho}^{\mu} C^{\rho}$,

where

$$
\begin{aligned}
X_{\rho}^{\mu}= & \left(\bar{g}^{\mu \nu} \bar{\square}+\beta \bar{\nabla}^{\mu} \bar{\nabla}^{v}\right)\left[\bar{\nabla}_{\rho} \bar{\nabla}_{v}+\bar{g}_{\nu \rho} \bar{\square}-\frac{2(1+\rho)}{d} \bar{\nabla}_{\nu} \bar{\nabla}_{\rho}\right. \\
& +\bar{\nabla}_{\rho} h_{\sigma v} \bar{\nabla}^{\sigma}+\bar{\nabla}^{\sigma} \bar{\nabla}_{\rho} h_{\sigma v}+\bar{\nabla}^{\sigma} h_{v \rho} \bar{\nabla}_{\sigma} \\
& +h_{v \rho} \bar{\square}+\bar{\nabla}^{\sigma} h_{\sigma \rho} \bar{\nabla}_{v}+h_{\sigma \rho} \bar{\nabla}^{\sigma} \bar{\nabla}_{v} \\
& -\frac{1+\rho}{d}\left(\bar{\nabla}_{\rho} h \bar{\nabla}_{v}+\bar{\nabla}_{v} \bar{\nabla}_{\rho} h\right. \\
& \left.\left.+2 \bar{\nabla}_{\nu} h_{\sigma \rho} \bar{\nabla}^{\sigma}+2 h_{\sigma \rho} \bar{\nabla}_{v} \bar{\nabla}^{\sigma}\right)\right] .
\end{aligned}
$$

Here the last four lines contain terms linear in $h_{\mu \nu}$. These are not relevant in doing one-loop computations, but at higher loops they are important. In the following we will ignore ghost contributions completely as they are not relevant in the computation of the running of the matter couplings, while the running of gravitational couplings are taken from past literature $[5,6,16,26,78]$.

\subsection{Gravitational field propagator}

The propagator for the gravitational field is obtained by expanding the gravitational action around the flat space-time up to second order in the fluctuation field $h_{\mu \nu}$. By decomposing the fluctuation field in terms of various components and writing them using the projection operators $\left(P_{2}^{\mu \nu \rho \sigma}, P_{1}^{\mu \nu \rho \sigma}\right.$, $P_{s}^{\mu \nu \rho \sigma}, P_{s w}^{\mu \nu \rho \sigma}, P_{w s}^{\mu \nu \rho \sigma}$ and $\left.P_{w}^{\mu \nu \rho \sigma}\right)$ as given in Appendix B, we note that this second variation can be expressed in a neat form in momentum space in the following manner:

$\delta^{2} S_{\mathrm{GR}}=\frac{1}{32 \pi} \int \frac{\mathrm{d}^{d} q}{(2 \pi)^{d}} h_{\mu \nu}\left[-\frac{q^{4}}{2 f^{2}} P_{2}^{\mu \nu \rho \sigma}+\frac{q^{4} \omega}{f^{2}} P_{s}^{\mu \nu \rho \sigma}\right] h_{\rho \sigma}$.

Moreover, the gauge-fixing action can be similarly expressed by using the projection operators,

$$
\begin{aligned}
S_{\mathrm{GF}}= & \frac{1}{32 \pi \alpha} \int \frac{\mathrm{d}^{d} q}{(2 \pi)^{d}} h_{\mu \nu} q^{4}\left[-\frac{1}{2} P_{1}^{\mu \nu \rho \sigma}+\frac{1-\beta}{d^{2}}\right. \\
& \times\left\{(1+\rho)^{2}(d-1) P_{s}^{\mu \nu \rho \sigma}+(d-1-\rho)^{2} P_{w}^{\mu \nu \rho \sigma}\right. \\
& \left.\left.-\sqrt{d-1}(1+\rho)(d-1-\rho)\left(P_{s w}^{\mu \nu \rho \sigma}+P_{w s}^{\mu \nu \rho \sigma}\right)\right\}\right] h_{\rho \sigma} .
\end{aligned}
$$

By writing the gauge-fixing action in terms of the projection operators allows us to see clearly which modes of the field are affected by the gauge fixing. For example the spin2 mode is not affected at all by the gauge-fixing condition. Interestingly it should be noted that there is another gaugeinvariant mode of the field which arises due to the action of spin-s projection operator on the $h_{\mu \nu}$ field (see Appendix B). However, under harmonic type gauge-fixing condition this mode does not remain completely unaffected. Only for some particular gauge choices this mode is not affected by the gauge-fixing condition. Landau gauge being one such choice $\rho=-1, \beta=0, \alpha=0$. In this gauge choice only the purely longitudinal modes are gauge fixed. In this gauge the propagator for the metric fluctuation field is the following:

$$
\begin{aligned}
D^{\mu \nu \rho \sigma} & =\left(\Delta_{G}^{-1}\right)^{\mu \nu \rho \sigma}=(16 \pi) \frac{f^{2}}{q^{4}}\left(-2 P_{2}^{\mu \nu \rho \sigma}+\frac{1}{\omega} P_{s}^{\mu \nu \rho \sigma}\right) \\
& =\sum_{i} Y_{i}\left(q^{2}\right) P_{i}^{\mu \nu \rho \sigma},
\end{aligned}
$$

where $Y_{i}$ are the propagators for the various spin components:

$Y_{2}=-(16 \pi) \frac{2 f^{2}}{q^{4}} \quad Y_{s}=(16 \pi) \frac{f^{2}}{\omega q^{4}}$.

Here $\Delta_{G}^{\mu \nu \alpha \beta}$ is the inverse propagator for the $h_{\mu \nu}$ field including the gauge fixing and is symmetric in $\mu \nu$ and $\alpha \beta$. As the propagator is $1 / q^{4}$, it is not allowed to be decomposed further via partial fractions. Here the first term in Eq. (17) arises due to the presence of the $C_{\mu \nu \rho \sigma}^{2}$ part of action, while the latter comes from the $R^{2}$ part. In this form it is not clear how the unitarity will be satisfied.

\subsection{Formalism}

We employ the background field formalism and decompose the metric and matter fields as in Eqs. (5) and (6) respectively, where we choose the background space-time to be flat. In order to do the one-loop computation the action is expanded up to second powers of the whole fluctuation field $\left(h_{\mu \nu}, \chi\right.$, $\eta$ and $\bar{\eta}$ ). This will result in various vertices and propagators that are required for the one-loop analysis. The second variation of the matter action is given by the following:

$$
\begin{aligned}
\delta^{2} S_{\text {matter }}=\frac{1}{2} \int \mathrm{d}^{d} x\left[\left(\frac{1}{4} h^{2}-\frac{1}{2} h_{\mu \nu} h^{\mu \nu}\right)\right. \\
\quad \times\left\{\frac{1}{2} \partial_{\alpha} \varphi \partial^{\alpha} \varphi-\frac{\lambda}{4} \varphi^{4}\right\}-\frac{1}{2} h h^{\mu \nu} \partial_{\mu} \varphi \partial_{\nu} \varphi+h^{\mu \alpha} h_{\alpha}{ }^{\nu} \partial_{\mu} \varphi \partial_{\nu} \varphi \\
\quad-\frac{1}{2} \xi \varphi^{2}\left(h \partial_{\mu} \partial_{\nu} h^{\mu \nu}-h \square h-4 h^{\mu \nu} \partial_{\mu} \partial_{\rho} h_{\nu}^{\rho}\right. \\
+2 h^{\mu \nu} \square h_{\mu \nu}+2 h^{\mu \nu} \partial_{\mu} \partial_{\nu} h-2 \partial_{\rho} h^{\rho \sigma} \partial^{\mu} h_{\sigma \mu} \\
+2 \partial_{\rho} h^{\rho \sigma} \partial_{\sigma} h+\frac{3}{2} \partial_{\mu} h^{\rho \sigma} \partial^{\mu} h_{\rho \sigma} \\
\left.\quad-\frac{1}{2} \partial_{\mu} h \partial^{\mu} h-\partial^{\sigma} h^{\rho \mu} \partial_{\rho} h_{\sigma \mu}\right) \\
\quad-\lambda \varphi^{3} h \chi+h \partial_{\mu} \varphi \partial^{\mu} \chi-2 h^{\mu \nu} \partial_{\mu} \varphi \partial_{\nu} \chi \\
\left.\quad-2 \xi \chi \varphi\left(\partial_{\mu} \partial_{\nu} h^{\mu \nu}-\square h\right)+\partial_{\mu} \chi \partial^{\mu} \chi-3 \lambda \varphi^{2} \chi^{2}\right] \\
\quad+\int \mathrm{d}^{d} x\left[\left\{\left(\frac{1}{8} h^{2}-\frac{1}{4} h_{\alpha \beta} h^{\alpha \beta}\right) \delta_{\rho}{ }^{\mu}-\frac{1}{4} h h_{\rho}{ }^{\mu}+\frac{3}{8} h_{\rho}{ }^{\alpha} h_{\alpha}{ }^{\mu}\right\}\right.
\end{aligned}
$$




$$
\begin{aligned}
& \times \bar{\theta} i \gamma^{\rho} \partial_{\mu} \theta+\frac{i}{4} \bar{\theta} \gamma^{\rho}\left[\gamma^{\alpha}, \gamma^{\beta}\right] \theta\left\{-\frac{1}{4} h_{\alpha}{ }^{\sigma} \partial_{\rho} h_{\beta \sigma}+\frac{1}{2} \partial_{\alpha}\left(h_{\beta \sigma} h_{\rho}^{\sigma}\right)\right. \\
& \left.+\frac{1}{2} h_{\alpha}{ }^{\sigma} \partial_{\sigma} h_{\beta \rho}-\frac{1}{2} h \partial_{\alpha} h_{\beta \rho}\right\}-y_{t} \varphi\left(\frac{1}{8} h^{2}-\frac{1}{4} h_{\alpha \beta} h^{\alpha \beta}\right) \\
& +\bar{\eta}\left\{\frac{i}{2} \gamma^{\rho}\left(h \delta_{\rho}{ }^{\mu}-h_{\rho}{ }^{\mu}\right) \partial_{\mu}-\frac{i}{4} \gamma^{\rho}\left[\gamma^{\alpha}, \gamma^{\beta}\right] \partial_{\alpha} h_{\beta \rho}\right. \\
& \left.-\frac{1}{2} y_{t} \varphi h\right\} \theta+\bar{\theta}\left\{\frac{i}{2} \gamma^{\rho}\left(h \delta_{\rho}^{\mu}-h_{\rho}{ }^{\mu}\right) \partial_{\mu}-\frac{i}{4} \gamma^{\rho}\left[\gamma^{\alpha}, \gamma^{\beta}\right] \partial_{\alpha} h_{\beta \rho}\right. \\
& \left.-\frac{1}{2} y_{t} \varphi h\right\} \eta+\bar{\eta}\left(i \gamma^{\rho} \partial_{\rho}-y_{t} \varphi\right) \eta \\
& \left.-y_{t}\left(\chi \bar{\eta} \theta+\chi \bar{\theta} \eta+\frac{1}{2} h \chi \bar{\theta} \theta\right)\right] .
\end{aligned}
$$

The various vertices and matter propagators are written in detail in Appendix C. Having obtained the second variation giving the propagator and the vertices, we proceed by considering the path integral over the fluctuation fields. In this case the zeroth-order term will be independent of the fluctuation fields and can be taken out of the path integral. The linear term can be removed by a field redefinition. In general, terms proportional to the equation of motion can be removed by a field redefinition. Such a redefinition will give rise to a trivial jacobian from the functional measure. The quadratic piece can now be investigated easily by putting together all the field fluctuations in the form of a multiplet, $\Phi^{\mathrm{T}}=\left(h_{\mu \nu}, \chi, \eta^{\mathrm{T}}, \bar{\eta}\right)$. Using this the path integral can be written in a more compact form,

$$
\begin{aligned}
& Z[\mathbf{J}]=\exp \left[i\left(S_{\mathrm{GR}}(\bar{g})+S_{\text {matter }}(\varphi, \bar{\theta}, \theta)\right)\right] \\
& \times \int \mathcal{D} \Phi \exp \left[i \int \mathrm{d}^{d} x\left(\frac{1}{2} \Phi^{\mathrm{T}} \cdot \mathbf{M} \cdot \Phi+\Phi^{\mathrm{T}} \cdot \mathbf{J}\right)\right],
\end{aligned}
$$

where $\mathbf{J}=\left\{t_{\mu \nu}, t, \rho, \bar{\rho}^{\mathrm{T}}\right\}$ is the source multiplet which couples with the fluctuation field multiplet $\Phi=\left(h_{\mu \nu}, \chi, \eta^{\mathrm{T}}, \bar{\eta}\right)$. The super-matrix $\mathbf{M}$ is given by
$\Gamma^{1 \text {-loop }}[\Phi]=S_{\mathrm{GR}}(\bar{g})+S_{\text {matter }}(\varphi, \bar{\theta}, \theta)+\frac{i}{2} \mathrm{~S} \operatorname{Tr} \ln \mathbf{M}$,

where the first two terms correspond to tree-level diagrams while the last term contains one-loop quantum corrections. The appearance of the generalised trace 'STr' means that

$\operatorname{STr}\left(\begin{array}{ll}a & \alpha \\ \beta & b\end{array}\right)=\operatorname{Tr}(a)-\operatorname{Tr}(b)$.

In the following we will compute the divergent pieces present in the $\mathrm{STr} \ln \mathbf{M}$. There are various ways to compute the oneloop quantum corrections. The most common methodology to do is via Feynman diagrams after computing vertices and propagator. Here we will follow a slightly different strategy of computation via evaluation of functional determinant. We start by writing $\mathbf{M}=\Delta+\mathbf{V}$, where the former $\Delta$ contains the various propagator while the latter $\mathbb{V}$ contains various vertices. They are given by

$$
\begin{aligned}
& \Delta=\left[\begin{array}{cccc}
\Delta_{G}^{\mu \nu \rho \sigma} & 0 & 0 & 0 \\
0 & \Delta_{S} & 0 & 0 \\
0 & 0 & 0 & \left(\Delta_{F}^{\mathrm{T}}\right)_{a b} \\
0 & 0 & \left(\Delta_{F}\right)_{a b} & 0
\end{array}\right] \text {, } \\
& \mathbf{V}=\left[\begin{array}{cccc}
V^{\mu \nu \rho \sigma}+U^{\mu \nu \rho \sigma} & V_{h \phi}^{\mu \nu} & \left(V_{h \psi}^{\mu \nu}\right)_{b} & \left(V_{h \bar{\psi}}^{\mathrm{T}}\right)_{b}^{\mu \nu} \\
V_{\phi h}^{\rho \sigma} & -V_{S} & \left(V_{\phi \psi}\right)_{b} & \left(V_{\phi \bar{\psi}}^{\mathrm{T}}\right)_{b} \\
\left(V_{\bar{\psi} h}^{\mathrm{T}}\right)_{a}^{\rho \sigma} & \left(V_{\bar{\psi} \phi}^{\mathrm{T}}\right)_{a} & 0 & \left(V_{\bar{\psi} \psi}^{\mathrm{T}}\right)_{a b} \\
\left(V_{\bar{\psi} h}^{\rho \sigma}\right)_{a} & \left(V_{\bar{\psi} \phi}\right)_{a} & -\left(V_{\bar{\psi} \psi}\right)_{a b} & 0
\end{array}\right]
\end{aligned}
$$

Pulling out $\Delta$ from the expression for $\mathbf{M}$ allows one to expand the residual expression $\left(\mathbf{I}+\Delta^{-1} \cdot \mathbf{V}\right.$ ) (where $\mathbf{I}$ is a generalised identity in super-field space) under the logarithm in a perturbative manner as follows:

$$
\mathbf{M}=\left[\begin{array}{cccc}
\Delta_{G}^{\mu \nu \rho \sigma}+V^{\mu \nu \rho \sigma}+U^{\mu \nu \rho \sigma} & V_{h \phi}^{\mu \nu} & \left(V_{h \psi}^{\mu \nu}\right)_{b} & \left(V_{h \bar{\psi}}^{\mathrm{T}}\right)_{b}^{\mu \nu} \\
V_{\phi h}^{\rho \sigma} & \Delta_{s}-V_{s} & \left(V_{\phi \psi}\right)_{b} & \left(V_{\phi \bar{\psi}}^{\mathrm{T}}\right)_{b} \\
\left(V_{\psi h}^{\mathrm{T}}\right)_{a}^{\rho \sigma} & \left(V_{\bar{\psi} \phi}^{\mathrm{T}}\right)_{a} & 0 & \left(\Delta_{F}^{\mathrm{T}}\right)_{a b}+\left(V_{\bar{\psi} \psi}^{\mathrm{T}}\right)_{a b} \\
\left(V_{\bar{\psi} h}^{\rho \sigma}\right)_{a} & \left(V_{\bar{\psi} \phi}\right)_{a}\left(\Delta_{F}\right)_{a b}-\left(V_{\bar{\psi} \psi}\right)_{a b} & 0
\end{array}\right] .
$$

From the generating functional $Z$, one can define the oneparticle-irreducible (1PI) generating functional $\Gamma=W[\mathbf{J}]-$ $\int \mathrm{d}^{d} x\left\langle\Phi^{\mathrm{T}}\right\rangle \cdot \mathbf{J}$, where $W[\mathbf{J}]=-i \ln Z[\mathbf{J}]$ and $\left\langle\Phi^{\mathrm{T}}\right\rangle$ is the expectation value of $\Phi^{\mathrm{T}}$ field. The 1PI generating functional is also the effective action containing the quantum corrections. In the one-loop approximation (which we are considering here), one can perform the functional integral over the super-field $\Phi$ thereby giving an expression for the one-loop effective action to be,

$$
\begin{aligned}
& \mathrm{S} \operatorname{Tr} \ln \mathbf{M}=\mathrm{S} \operatorname{Tr} \ln \Delta \cdot(\mathbf{I}+\mathbb{V}) \\
& \quad=\mathrm{S} \operatorname{Tr} \ln \Delta+\mathrm{S} \operatorname{Tr}\left[\mathbb{V}-\frac{1}{2} \mathbb{V}^{2}+\frac{1}{3} \mathbb{V}^{3}-\frac{1}{4} \mathbb{V}^{4}+\cdots\right] .
\end{aligned}
$$

Here $\mathbb{V}=\Delta^{-1} \cdot \mathbf{V}$ is given by 


$$
\mathbb{V}=\left[\begin{array}{cccc}
\left(\Delta_{G}^{-1}\right)^{\mu \nu \rho \sigma}(V+U)_{\rho \sigma \alpha \beta} & \left(\Delta_{G}^{-1}\right)^{\mu \nu \rho \sigma}\left(V_{h \phi}\right)_{\rho \sigma} & \left(\Delta_{G}^{-1}\right)^{\mu \nu \rho \sigma}\left(V_{h \psi}\right)_{c \rho \sigma} & \left(\Delta_{G}^{-1}\right)^{\mu \nu \rho \sigma}\left(V_{h \bar{\psi}}^{\mathrm{T}}\right)_{c \rho \sigma} \\
\Delta_{s}^{-1}\left(V_{\phi h}\right)_{\alpha \beta} & -\Delta_{s}^{-1} V_{s} & \Delta_{s}^{-1}\left(V_{\phi \psi}\right)_{c} & \Delta_{s}^{-1}\left(V_{\phi \bar{\psi}}^{\mathrm{T}}\right)_{c} \\
\left(\Delta_{F}^{-1}\right)_{a b}\left(V_{\bar{\psi} h}\right)_{b \alpha \beta} & \left(\Delta_{F}^{-1}\right)_{a b}\left(V_{\bar{\psi} \phi}\right)_{b} & -\left(\Delta_{F}^{-1}\right)_{a b}\left(V_{\bar{\psi} \psi}\right)_{b c} & 0 \\
\left(\Delta_{F}^{-1}\right)_{a b}^{\mathrm{T}}\left(V_{\psi h}^{\mathrm{T}}\right)_{b \alpha \beta} & \left(\Delta_{F}^{-1}\right)_{a b}^{\mathrm{T}}\left(V_{\psi \phi}^{\mathrm{T}}\right)_{b} & 0 & \left(\Delta_{F}^{-1}\right)_{a b}^{\mathrm{T}}\left(V_{\bar{\psi} \psi}^{\mathrm{T}}\right)_{b c}
\end{array}\right] .
$$

It should be mentioned here that so far we took background metric to be flat with $H_{\mu \nu}=0$. This is enough to compute the counter-terms involving quantum gravity corrections to all matter couplings including their anomalous dimensions. If we had included terms with $H_{\mu \nu} \neq 0$, then it is also possible to compute the counter-term proportional to $R \varphi^{2}$. But here for simplicity we keep $H_{\mu v}=0$, and the counter-term proportional to $R \varphi^{2}$ will be computed using methodology of heat kernels (HKs) later. The heat-kernel method is quick, as the HK coefficients have already been computed in past [7578]. Besides, it also gives an alternative check on the computation done using Feynman diagrams. For a flat background the term $\operatorname{STr} \ln \Delta$ is irrelevant, but it is not so if the background is non-flat, for which case this gives purely curvaturedependent divergent contributions. Such contributions have been computed elsewhere in the literature $[16,78]$ and here we will just take the results.

In the following we will compute the various graphs that are giving quantum gravity corrections to the running of the matter couplings and the fields anomalous dimensions.

\subsection{Graphs}

Here we will compute the various graphs that contain divergent contributions. These are basically the terms in the series expansion given in Eq. (25), which will be evaluated one by one. The first term of the series contains tadpole graphs (those having single vertex), the second term of series has bubble graphs (those having two vertices), the third term of the series are triangle graphs containing three vertices while the fourth term of series are square graphs with four vertices. The series has an infinite number of graphs, but the divergent ones are only present in the first four terms of the series expansion, and below we will compute them.

\subsubsection{Tadpole}

These set of graphs arises from the first term of the series in Eq. (25) which is $\operatorname{STr}(\mathbb{V})=\operatorname{STr}\left(\Delta^{-1} \cdot \mathbf{V}\right)$. Here the supertrace takes care of the trace over bosonic and fermionic part, and includes the trace not only over field space but also over Lorentz indices. This will imply the following,
$\mathrm{STr} \mathbb{V}=\operatorname{Tr}\left[\mathbb{V}_{11}+\mathbb{V}_{22}-\mathbb{V}_{33}-\mathbb{V}_{44}\right]$

$$
\begin{aligned}
= & \operatorname{Tr}\left[\left(\Delta_{G}^{-1}\right)^{\mu \nu \rho \sigma}(V+U)_{\rho \sigma \alpha \beta}-\Delta_{s}^{-1} V_{s}\right. \\
& \left.+\left(\Delta_{F}^{-1}\right)_{a b}\left(V_{\bar{\psi} \psi}\right)_{b c}-\left(\Delta_{F}^{-1}\right)_{a b}^{\mathrm{T}}\left(V_{\bar{\psi} \psi}^{\mathrm{T}}\right)_{b c}\right] .
\end{aligned}
$$

Here the first term contains graphs having an internal graviton line, while the next three terms contains the usual diagrams which are present without gravity. The former gives quantum gravity contribution. The set of graphs present in the tadpole order are shown in Fig. 1.

Each of these diagrams can be evaluated using the vertices given in Appendix C.2. Here we will write their contribution. However, the last three terms in Eq. (27) vanish in scaleinvariant theory. The gravitational ones are complicated and lengthy as the vertices are cumbersome. We write

$$
\begin{aligned}
& \operatorname{Tr}\left\{\left(\Delta_{G}^{-1}\right)^{\mu \nu \rho \sigma} V_{\rho \sigma \alpha \beta}\right\}=\frac{1}{4} \int \mathrm{d}^{d} x\left[\frac{d-4}{2 d}(\partial \varphi)^{2}-\frac{\lambda}{4} \varphi^{4}\right] \\
& \quad \times \int \frac{\mathrm{d}^{d} p}{(2 \pi)^{d}}\left\{-(d-2)(d+1) Y_{2}+(d-3) Y_{s}\right\} \\
& +\frac{(d-2) \xi}{8} \int \mathrm{d}^{d} x \varphi^{2} \int \frac{\mathrm{d}^{d} p}{(2 \pi)^{d}} p^{2}\left\{(d+1) Y_{2}-2 Y_{s}\right\} .
\end{aligned}
$$

For the other one there is more algebra as it involves Dirac matrices. Here we will write the expression after performing the Lorentz and Dirac matrix algebra. This is given by

$$
\begin{aligned}
& \operatorname{Tr}\left\{\left(\Delta_{G}^{-1}\right)^{\mu \nu \rho \sigma} U_{\rho \sigma \alpha \beta}\right\}=\frac{(d-2)(d+1)}{4} \\
& \quad \times \int \mathrm{d}^{d} x\left[\frac{3-2 d}{2 d} \bar{\theta} i \gamma^{\alpha} \partial_{\alpha} \theta+y_{t} \varphi \bar{\theta} \theta\right] \\
& \quad \times \int \frac{\mathrm{d}^{d} p}{(2 \pi)^{d}} Y_{2}+\int \mathrm{d}^{d} x\left[\frac{d^{2}-5 d+5}{4 d} \bar{\theta} i \gamma^{\alpha} \partial_{\alpha} \theta\right. \\
& \left.-\frac{d-3}{4} y_{t} \varphi \bar{\theta} \theta\right] \int \frac{\mathrm{d}^{d} p}{(2 \pi)^{d}} Y_{s} .
\end{aligned}
$$

The momentum integrals can be evaluated in the $(4-\epsilon)$ dimensional regularisation scheme and the divergent piece can be singled out easily. The divergent piece of all the above tadpole contribution is, 


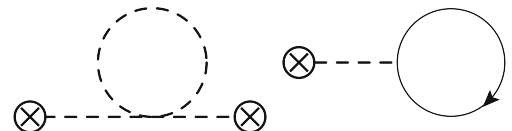

$\otimes-\infty$
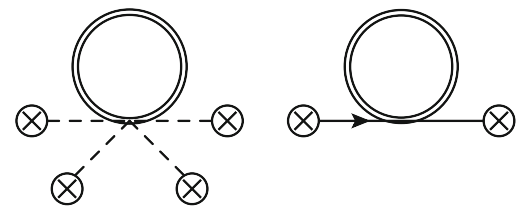

Fig. 1 Various diagrams containing divergences at the tadpole level. Here the dashed line represents the scalar field, solid line with arrow represents fermion field, while a double line represent $h_{\mu \nu}$-field. The

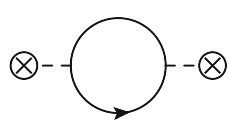<smiles>O=C1CCC(O)(O)CCC1(O)O</smiles><smiles>OC1CCCCCCC1(O)O</smiles>

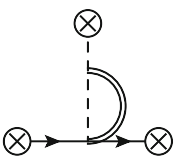

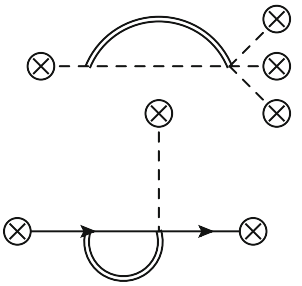

Fig. 2 Various diagrams containing divergences with two vertices. Here the dashed line represents the scalar field, the solid line with arrow represents fermion field, while the double line represents the $h_{\mu \nu}$-field. Here the graphs on the first line are purely matter ones. The second and third line contain graphs having quantum gravity corrections

$$
\begin{aligned}
\Gamma_{\text {div }}^{\mathrm{Tad}} & =-\frac{\mu^{\epsilon}}{16 \pi^{2} \epsilon} \frac{M^{2}}{Z}\left[\frac{\lambda}{8}\left(10+\frac{1}{2 \omega}\right) \int \mathrm{d}^{d} x \varphi^{4}\right. \\
& -\left(\frac{25}{8}+\frac{1}{16 \omega}\right) \int \mathrm{d}^{d} x \bar{\theta}\left(i \gamma^{\mu} \partial_{\mu}\right) \theta \\
& \left.+5 y_{t}\left(1+\frac{1}{4 \omega}\right) \int \mathrm{d}^{d} x \bar{\theta} \varphi \theta\right],
\end{aligned}
$$

where $M^{2} / Z=16 \pi f^{2}$ is introduced for convenience.

\subsubsection{Bubble}

These sets of graphs arise from second term in Eq. (25), which is $-1 / 2 \operatorname{STr}\left(\Delta^{-1} \cdot \mathbf{V}\right)^{2}$. Here again the super-trace is evaluated as before. This will imply

$\mathrm{S} \operatorname{Tr} \mathbb{V}^{2}=\operatorname{Tr}\left[\left(\mathbb{V}^{2}\right)_{11}+\left(\mathbb{V}^{2}\right)_{22}-\left(\mathbb{V}^{2}\right)_{33}-\left(\mathbb{V}^{2}\right)_{44}\right]$

Here each of the terms will contain several diagrams, but only few contain the divergences that are relevant for our purposes. These diagrams contain two vertices. They can be classified in three categories: (a) those with two internal graviton lines, (b) those with one internal graviton and one internal matter line and (c) those with two internal matter lines. The set of diagrams are shown in Fig. 2. first two graphs are purely matter ones, while the other four graphs contain quantum gravity corrections

Each of these diagrams can be evaluated using the vertices given in Appendix C.2. The super-trace given in Eq. (31) contains a lot of diagrams, but not all contain a UV divergence. Here we will mention only the ones having UV divergences. These come from

$$
\begin{aligned}
\operatorname{Tr} & \Delta_{s}^{-1} V_{s} \Delta_{s}^{-1} V_{s}+2 \Delta_{s}^{-1}\left(V_{\phi \psi}\right)_{c}\left(\Delta_{F}^{-1}\right)_{c d}\left(V_{\bar{\psi} \phi}\right)_{d} \\
& +2 \Delta_{s}^{-1}\left(V_{\phi \psi}^{\mathrm{T}}\right)_{c}\left(\Delta_{F}^{-1}\right)_{c d}^{\mathrm{T}}\left(V_{\psi \phi}^{\mathrm{T}}\right)_{d} \\
& -\left(D_{F}^{-1}\right)_{a b}\left(V_{\bar{\psi} \psi}\right)_{b d}\left(\Delta_{F}^{-1}\right)_{d e}\left(V_{\bar{\psi} \psi}\right)_{e c} \\
& -\left(D_{F}^{-1}\right)_{a b}^{\mathrm{T}}\left(V_{\bar{\psi} \psi}^{\mathrm{T}}\right)_{b d}\left(\Delta_{F}^{-1}\right)_{d e}^{\mathrm{T}}\left(V_{\bar{\psi} \psi}^{\mathrm{T}}\right)_{e c} \\
& +\left(\Delta_{G}^{-1}\right)^{\mu \nu \rho \sigma} V_{\rho \sigma \alpha \beta}\left(\Delta_{G}^{-1}\right)^{\alpha \beta \theta \tau} V_{\theta \tau \mu^{\prime} \nu^{\prime}} \\
& +2\left(\Delta_{G}^{-1}\right)^{\mu \nu \rho \sigma}\left(V_{h \phi}\right)_{\rho \sigma} \Delta_{s}^{-1}\left(V_{\phi h}\right)_{\mu^{\prime} v^{\prime}} \\
& +2\left(\Delta_{G}^{-1}\right)^{\mu \nu \rho \sigma}\left(V_{h \psi}\right)_{\rho \sigma c}\left(\Delta_{F}^{-1}\right)_{c d}\left(V_{\bar{\psi} h}\right)_{d \mu^{\prime} v^{\prime}} \\
& \left.+2\left(\Delta_{G}^{-1}\right)^{\mu \nu \rho \sigma}\left(V_{h \bar{\psi}}^{\mathrm{T}}\right)_{c \rho \sigma}\left(\Delta_{F}^{-1}\right)_{c d}^{\mathrm{T}}\left(V_{\psi h}^{\mathrm{T}}\right)_{d \mu^{\prime} \nu^{\prime}}\right] .
\end{aligned}
$$

From the various terms written in Eq. (32) the first two lines contain diagrams which are purely matter ones and correspond to the diagrams shown in first row of Fig. 2, while the last two lines contain diagrams having quantum contributions and correspond to the diagrams shown in last two rows of the Fig. 2. The trace is over the Lorentz and space-time indices. After performing the algebra over Dirac matrices and doing the contraction of the tensors we get the simplified expression involving momentum integrals. The divergent contributions of the purely matter diagrams are

$\frac{i}{16 \pi^{2}} \frac{1}{\epsilon}\left[9 \lambda^{2} \int \mathrm{d}^{4} x \varphi^{4}+2 y_{t}^{2} \int \mathrm{d}^{4} x\left\{\bar{\theta} i \not \partial \theta+2 \partial_{\mu} \varphi \partial^{\mu} \varphi\right\}\right]$.

The diagrams containing the internal graviton legs are a bit complicated, as it involves lengthy Dirac matrix algebra and tensor manipulations. For doing these we have used various tricks to extract the divergent piece and also used MATHEMATICA packages (xAct [79], xTras [80] and FEYNCALC [81]). Below for simplicity we will mention only the divergent piece of these diagrams to evade unnecessary complications, while some of the details will be mentioned in the appendix. The diagrams with an internal graviton line has the following contributions: 

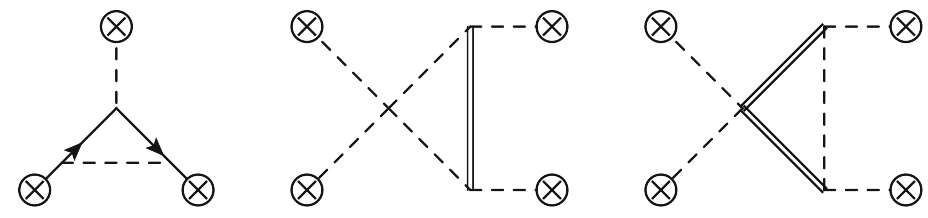
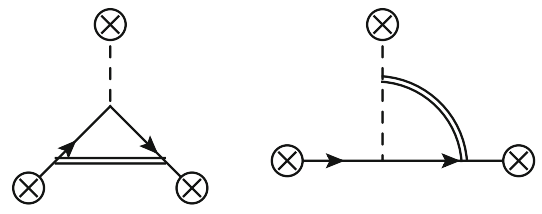

Fig. 3 Various diagrams containing divergences with three vertices. Here the first graph is purely matter oriented and gives correction to Yukawa coupling. The next two diagrams give a correction to $\varphi^{4}$ cou- pling. They are only present in the quantum gravity context. The last two diagrams give a quantum gravity correction to the Yukawa coupling

terms in the trace which will be of relevance can be guessed by looking at the set of third-order diagrams in Fig. 3. These are given by

$$
\begin{aligned}
& +\frac{3}{8} \frac{M^{2}}{Z \omega} \int \mathrm{d}^{4} x \partial_{\mu} \varphi \partial^{\mu} \varphi+\frac{M^{2}}{Z \omega} \\
& \left.\times\left\{\frac{9}{4} \int \mathrm{d}^{4} x \bar{\theta} i \not \partial \theta+3(2 \xi-1) y_{t} \int \mathrm{d}^{4} x \varphi \bar{\theta} \theta\right\}\right] .
\end{aligned}
$$

Here the first row contains contributions to the scalar sector, while the second and third row contain contributions to the fermion sector. The former correspond to diagrams of the second row in Fig. 2, while the latter correspond to diagrams in the third row of Fig. 2, respectively.

Putting together the full contribution of the bubble kind of diagrams, we get contribution to the one-loop effective action of the diagrams having two vertices. This is given by

$$
\begin{aligned}
\Gamma_{\mathrm{div}}^{\mathrm{Bub}}=- & \frac{1}{16 \pi^{2}} \frac{1}{\epsilon}\left[\left\{2 y_{t}^{2}+\frac{3 M^{2}}{16 Z \omega}\right\} \int \mathrm{d}^{4} x \partial_{\mu} \varphi \partial^{\mu} \varphi\right. \\
& +\left\{\frac{9 \lambda^{2}}{2}+\frac{3 \xi \lambda M^{2}}{Z \omega}+\frac{1}{2}\left(\frac{\xi M^{2}}{2 Z}\right)^{2}\left(5+\frac{1}{\omega^{2}}\right)\right\} \\
& \times \int \mathrm{d}^{4} x \varphi^{4}+\left(y_{t}^{2}+\frac{9 M^{2}}{8 Z \omega}\right) \int \mathrm{d}^{4} x \bar{\theta} i \not \partial \theta \\
& \left.+\frac{3(2 \xi-1) y_{t}}{2} \frac{M^{2}}{Z \omega} \int \mathrm{d}^{4} x \varphi \bar{\theta} \theta\right] .
\end{aligned}
$$

\subsubsection{Triangular graphs}

These diagram are generated from the third-order terms in the series of Eq. (25), 1/3STr $\mathbb{V}^{3}$ where

$\mathrm{S} \operatorname{Tr} \mathbb{V}^{3}=\operatorname{Tr}\left[\left(\mathbb{V}^{3}\right)_{11}+\left(\mathbb{V}^{3}\right)_{22}-\left(\mathbb{V}^{3}\right)_{33}-\left(\mathbb{V}^{3}\right)_{44}\right]$.

These diagrams have three vertices and consist of graphs which are either purely matter oriented or ones which include a mixture of matter and gravity. These graphs give correction to vertex: either to $\varphi^{4}$ or to Yukawa vertex $\varphi \bar{\theta} \theta$. On expansion we will see that there are many diagrams but we will consider those which carry divergent pieces and give a correction to the running couplings. Here we will only mention the trace pieces which will be carrying the divergences, however, in principle there will be many more diagrams. The relevant

$$
\begin{aligned}
3 \operatorname{Tr} & {\left[\Delta_{s}^{-1}\left(V_{\phi \bar{\psi}}^{\mathrm{T}}\right)_{a}\left(\Delta_{F}^{-1}\right)_{a b}^{\mathrm{T}}\left(V_{\bar{\psi} \psi}^{\mathrm{T}}\right)_{b c}\left(\Delta_{F}^{-1}\right)_{c d}^{\mathrm{T}}\left(V_{\psi \phi}^{\mathrm{T}}\right)_{d}\right.} \\
& -\Delta_{s}^{-1}\left(V_{\phi \psi}\right)_{a}\left(D_{F}^{-1}\right)_{a b}\left(V_{\bar{\psi} \psi}\right)_{b c}\left(\Delta_{F}^{-1}\right)_{c d}\left(V_{\bar{\psi} \phi}\right)_{d} \\
& +\left(\Delta_{G}^{-1}\right)^{\mu \nu \rho \sigma}\left\{\left(V_{h \phi}\right)_{\rho \sigma} \Delta_{s}^{-1}\left(V_{\phi h}\right)^{\alpha \beta}\left(\Delta_{G}^{-1}\right)_{\alpha \beta \theta \tau} V^{\theta \tau \gamma \delta}\right. \\
& -\left(V_{h \phi}\right)_{\rho \sigma} \Delta_{s}^{-1} V_{s} \Delta_{s}^{-1}\left(V_{\phi h}\right)^{\alpha \beta} \\
& +\left(V_{h \phi}\right)_{\rho \sigma} \Delta_{s}^{-1}\left(V_{\phi \psi}\right)_{a}\left(\Delta_{F}^{-1}\right)_{a b}\left(V_{\bar{\psi} h}\right)_{b \alpha \beta} \\
& +\left(V_{h \phi}\right)_{\rho \sigma} \Delta_{s}^{-1}\left(V_{\phi \bar{\psi}}^{\mathrm{T}}\right)_{a}\left(\Delta_{F}^{-1}\right)_{a b}^{\mathrm{T}}\left(V_{\psi h}^{\mathrm{T}}\right)_{b \mu \nu} \\
& +\left(V_{h \psi}\right)_{a \rho \sigma}\left(\Delta_{F}^{-1}\right)_{a b}\left(V_{\bar{\psi} \phi}\right)_{b} \Delta_{s}^{-1}\left(V_{\phi h}\right)_{\alpha \beta} \\
& +\left(V_{h \bar{\psi}}^{\mathrm{T}}\right)_{a \rho \sigma}\left(D_{F}^{-1}\right)_{a b}^{\mathrm{T}}\left(V_{\psi \phi}^{\mathrm{T}}\right)_{b} \Delta_{s}^{-1}\left(V_{\phi h}\right)_{\alpha \beta} \\
& -\left(V_{h \psi}\right)_{a \rho \sigma}\left(\Delta_{F}^{-1}\right)_{a b}\left(V_{\bar{\psi} \psi}\right)_{b c}\left(\Delta_{F}^{-1}\right)_{c d}\left(V_{h \psi}\right)_{d \alpha \beta} \\
& \left.\left.+\left(V_{h \bar{\psi}}^{\mathrm{T}}\right)_{a \rho \sigma}\left(\Delta_{F}^{-1}\right)_{a b}^{\mathrm{T}}\left(V_{\bar{\psi} \psi}^{\mathrm{T}}\right)_{b c}\left(\Delta_{F}^{-1}\right)_{c d}^{\mathrm{T}}\left(V_{\psi h}^{\mathrm{T}}\right)_{d \alpha \beta}\right\}\right] . \quad \text { (37) }
\end{aligned}
$$

Here the first and second line correspond to set of diagrams of purely matter type, while the rest of terms contain quantum gravity corrections. The second line contribute to running of $\varphi^{4}$ coupling while the rest of the terms correspond to the running of Yukawa coupling. Interestingly while doing the computation involving fermions it is noticed that not all of terms are non-zero. The divergent contributions of these diagrams and the corresponding contribution to the effective action are given by

$$
\begin{aligned}
\Gamma_{\text {div }}^{\text {Triangle }}= & -\frac{1}{16 \pi^{2}} \frac{1}{\epsilon} \int \mathrm{d}^{4} x\left[\left(2 y_{t}^{3}-\frac{3 M^{2} \xi}{Z \omega} y_{t}\right) \varphi \bar{\theta} \theta\right. \\
& \left.+\left(\frac{9 M^{2}}{Z \omega} \lambda \xi^{2}+\frac{3 M^{4}}{2 Z^{2} \omega^{2}} \xi^{3}\right) \varphi^{4}\right] .
\end{aligned}
$$

\subsubsection{Square graphs}

These set of contribution arise at fourth order of the perturbative expansion given in the series Eq. (25) and comes from $-1 / 4 \mathrm{~S} \operatorname{Tr} \mathbb{V}^{4}$. Here again the super-trace is given by,

$\mathrm{S} \operatorname{Tr} \mathbb{V}^{4}=\operatorname{Tr}\left[\left(\mathbb{V}^{4}\right)_{11}+\left(\mathbb{V}^{4}\right)_{22}-\left(\mathbb{V}^{4}\right)_{33}-\left(\mathbb{V}^{4}\right)_{44}\right]$. 

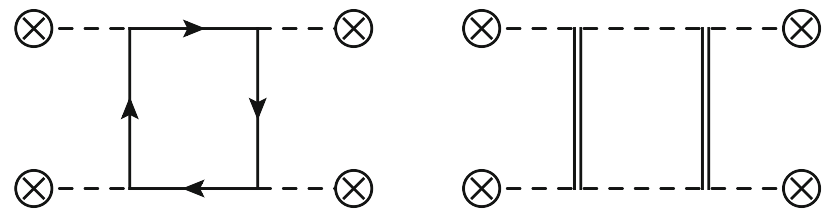

Fig. 4 Various diagrams containing divergences with four vertices. Here there are only two diagrams. The first one is purely matter, while the second one contains a quantum gravity correction. Both give corrections to the $\varphi^{4}$ coupling

These diagrams consist of four vertices and all of them contribute to the running of $\varphi^{4}$ coupling. There are only two diagrams at this order. One is of purely matter type and contains a fermion loop with four external scalar legs, while the other one contains quantum gravity correction. The trace can be expanded as before and consists of a large number of graphs but the ones containing the divergences are only two. These are the following:

$$
\begin{aligned}
2 \operatorname{Tr} & {\left[-\left(\Delta_{F}^{-1}\right)_{a b}\left(V_{\bar{\psi} \psi}\right)_{b c}\left(\Delta_{F}^{-1}\right)_{c d}\left(V_{\bar{\psi} \psi}\right)_{d e}\right.} \\
& \times\left(\Delta_{F}^{-1}\right)_{e f}\left(V_{\bar{\psi} \psi}\right)_{f g}\left(\Delta_{F}^{-1}\right)_{g k}\left(V_{\bar{\psi} \psi}\right)_{k l} \\
& +\left(\Delta_{G}^{-1}\right)^{\mu \nu \rho \sigma}\left(V_{h \phi}\right)_{\rho \sigma} \Delta_{s}^{-1}\left(V_{\phi h}\right)^{\alpha \beta} \\
& \left.\times\left(\Delta_{G}^{-1}\right)_{\alpha \beta \theta \tau}\left(V_{h \phi}\right)^{\theta \tau} \Delta_{s}^{-1}\left(V_{\phi h}\right)_{\gamma \delta}\right] .
\end{aligned}
$$

Here the former term is purely matter and contains a fermion loop, while the second term contains virtual gravitons (Fig. 4). Considering as before just the divergent part and the corresponding contribution to the effective action, we find that

$\Gamma_{\text {div }}^{\text {Square }}=-\frac{1}{\epsilon} \frac{1}{16 \pi^{2}}\left(-2 y_{t}^{4}+\frac{9 M^{4}}{2 Z^{2} \omega^{2}} \xi^{4}\right) \int \mathrm{d}^{4} x \varphi^{4}$.

\subsection{5 $R \varphi^{2}$ divergence}

Here we compute the divergence proportional to $R \varphi^{2}$. There are two ways to compute it. The first is via computation of Feynman graphs and second via heat kernel. Conceptually these are the same and give the same results, however, the latter is quicker. In each case we break the metric $\gamma_{\mu \nu}$ appearing in path integral is written as in Eq. (5). In the former the background is further written as $\eta_{\mu \nu}+H_{\mu \nu}$ (see also the paragraph following Eq. (5)). Here $H_{\mu \nu}$ will act as external graviton for the corresponding internal leg $h_{\mu \nu}$. The action is then expanded using this decomposition. This way we get additional vertices. The vertices in the previous section are the ones for which $H_{\mu v}=0$. If $H_{\mu v} \neq 0$ then we get contributions which contains dependence on external graviton leg, and if there are derivatives acting on $H_{\mu \nu}$, then those will give terms proportional to background curvature. This was employed in [26].
Alternatively, one can take the background metric $\bar{g}_{\mu \nu}$ to be maximally symmetric and compute the contributions proportional to background curvature using the heat-kernel formalism. This will project directly the contribution proportional to $\bar{R} \varphi^{2}$. We use the heat-kernel methodology to compute the one-loop divergence proportional to $\bar{R} \varphi^{2}$. The matter fields are decomposed as in Eq. (6), but this time we take the background matter fields to be constant. The fluctuation metric $h_{\mu \nu}$ is further decomposed into various components as

$h_{\mu \nu}=h_{\mu \nu}^{T T}+\bar{\nabla}_{\mu} \xi_{\nu}+\bar{\nabla}_{\nu} \xi_{\mu}+\bar{\nabla}_{\mu} \bar{\nabla}_{\nu} \sigma-\frac{1}{4} \bar{g}_{\mu \nu} \bar{\square} \sigma+\frac{1}{4} \bar{g}_{\mu \nu} h$,

where

$h_{\mu}^{T T \mu}=0, \quad \bar{\nabla}^{\mu} h_{\mu \nu}^{T T}=0, \quad \bar{\nabla}^{\mu} \xi_{\mu}=0$.

This decomposition of $h_{\mu \nu}$ introduces Jacobians in the path integral, which can be cancelled by redefining the fields as

$\hat{\xi}_{\mu}=\sqrt{-\bar{\square}-\frac{\bar{R}}{4}} \xi_{\mu}, \quad \hat{\sigma}=\sqrt{-\bar{\square}\left(-\bar{\square}-\frac{\bar{R}}{3}\right)} \sigma$.

With this decomposition the Hessian for the fluctuation fields is obtained on a maximally symmetric background. This will be same as in Eq. (20), except now $\mathbf{M}$ will be different. The multiplet $\Phi$ also gets modified $\Phi^{\mathrm{T}}=$ $\left(h_{\mu \nu}^{T T}, \hat{\xi}_{\mu}, \hat{\sigma}, h, \chi, \eta^{T}, \bar{\eta}\right)$. As the background matter fields are constant, the fermion sector is completely decoupled with mixing of fermion fluctuation field with metric and scalar fluctuations. This is not so when background matter is not constant. On the maximally symmetric background metric with constant background matter the matrix $\mathbf{M}$ is given by (in the Landau gauge $\rho=-1$ )

$$
\begin{aligned}
& \int \mathrm{d}^{d} x \sqrt{-\bar{g}} \Phi^{\mathrm{T}} \mathbf{M} \Phi=\int \mathrm{d}^{d} x \sqrt{-\bar{g}}\left[h_{\mu \nu}^{T T} \Delta_{2}\left(h^{T T}\right)^{\mu \nu}\right. \\
& +\hat{\xi}_{\mu} \Delta_{1} \hat{\xi}^{\mu}+\left(\begin{array}{lll}
\hat{\sigma} & h & \chi
\end{array}\right)\left(\begin{array}{ccc}
S_{\hat{\sigma} \hat{\sigma}} & S_{\hat{\sigma} h} & S_{\hat{\sigma} \phi} \\
S_{h \hat{\sigma}} & S_{h h} & S_{h \phi} \\
S_{\phi \hat{\sigma}} & S_{\phi h} & S_{\phi \phi}
\end{array}\right)\left(\begin{array}{c}
\hat{\sigma} \\
h \\
\chi
\end{array}\right) \\
& \left.+\frac{1}{2} \bar{\eta} \Delta_{1 / 2} \eta-\frac{1}{2} \eta^{\mathrm{T}} \Delta_{1 / 2}^{\mathrm{T}} \bar{\eta}^{\mathrm{T}}\right]
\end{aligned}
$$

where

$$
\begin{aligned}
\Delta_{2}= & \left\{-\frac{Z}{M^{2}} \bar{\square}^{2}+\left(-\xi \varphi^{2}+\frac{(3+2 \omega) Z \bar{R}}{3 M^{2}}\right) \bar{\square}\right. \\
& \left.+\left(\frac{\lambda \varphi^{4}}{8}+\frac{\xi \bar{R} \varphi^{2}}{6}+\frac{(1+\omega) Z \bar{R}^{2}}{36 M^{2}}\right)\right\}, \\
\Delta_{1}= & \left\{\frac{\square^{2}}{\alpha}+\frac{\bar{R} \bar{\square}}{4 \alpha}+\frac{\lambda \varphi^{4}+\xi \bar{R} \varphi^{2}}{4}\right\},
\end{aligned}
$$




$$
\begin{aligned}
S_{\hat{\sigma} \hat{\sigma}}= & {\left[\left(\frac{3}{\alpha}+\frac{Z \omega}{M^{2}}\right) \frac{3 \bar{\square}^{2}}{16}+\left(\frac{6 \alpha \xi \varphi^{2}+21 \bar{R}}{64 \alpha}\right) \bar{\square}\right.} \\
& \left.+\frac{3 \lambda \varphi^{4}}{32}+\frac{3 \xi \bar{R} \varphi^{2}}{32}+\frac{3 \bar{R}^{2}}{64 \alpha}\right], \\
S_{h \hat{\sigma}}= & S_{\hat{\sigma} h}=\sqrt{-\bar{\square}\left(-\bar{\square}-\frac{\bar{R}}{3}\right)} \\
& \times\left[\left(\frac{1}{\alpha}-\frac{Z \omega}{M^{2}}\right) \frac{3 \bar{\square}}{16}+\frac{3 \bar{R}-6 \alpha \xi \varphi^{2}}{64 \alpha}\right], \\
S_{\hat{\sigma} \phi}= & S_{\phi \hat{\sigma}}=-\frac{3 \xi \varphi}{4} \sqrt{-\bar{\square}\left(-\bar{\square}-\frac{\bar{R}}{3}\right),} \\
S_{h \phi}= & S_{\phi h}=\frac{\left(3 \xi \bar{\square}-\xi \bar{R}-2 \lambda \varphi^{2}\right) \varphi}{4}, \\
S_{h h}= & {\left[\left(\frac{1}{\alpha}+\frac{3 Z \omega}{M^{2}}\right) \frac{\bar{\square}}{16}+\left\{\left(\frac{1}{\alpha}+\frac{4 Z \omega}{M^{2}}\right)\right.\right.} \\
& \left.\left.\times \frac{\bar{R}}{64}+\frac{3}{32} \xi \varphi^{2}\right\} \bar{\square}-\frac{\lambda \varphi^{4}}{32}\right], \\
S_{\phi \phi}= & -\bar{\square}-\xi \bar{R}-3 \lambda \varphi^{2}, \\
\Delta_{1 / 2}= & \left(i \gamma_{1 / 2}^{\mu}=\right. \\
\left.\Delta_{\mu}-m-y_{t} \varphi\right), & \left(-i \gamma^{T} \bar{\nabla}_{\mu}-m-y_{t} \varphi\right) .
\end{aligned}
$$

We will not consider the contribution for the ghost here, as they will not contribute at one loop to the term proportional to $\bar{R} \varphi^{2}$. The one-loop effective action is given by

$$
\begin{aligned}
\Gamma^{(1)}= & \frac{(d+1)(d-2) i}{4} \operatorname{Tr} \ln \Delta_{2}+\frac{(d-1) i}{2} \operatorname{Tr} \ln \Delta_{1} \\
& +\frac{i}{2} \operatorname{Tr} \ln \left[\operatorname{det}\left(\begin{array}{lll}
S_{\hat{\sigma} \hat{\sigma}} & S_{\hat{\sigma} h} & S_{\hat{\sigma} \phi} \\
S_{h \hat{\sigma}} & S_{h h} & S_{h \phi} \\
S_{\phi \hat{\sigma}} & S_{\phi h} & S_{\phi \phi}
\end{array}\right)\right]-i \operatorname{Tr} \ln \Delta_{1 / 2} .
\end{aligned}
$$

These functional traces can be tackled using heat kernel [7578]. One can compute the divergent part of the effective action from this. Since the background matter fields are not constant, this will, however, not enable one to give the anomalous dimensions of the matter fields. However, the anomalous dimension has already been computed earlier using Feynman diagrams; therefore it will not be considered again here. Here we will just look at the divergent contribution proportional to $\bar{R} \varphi^{2}$, which is given by

$$
\begin{aligned}
& \Gamma_{\operatorname{div}}^{R \varphi^{2}}=-\frac{1}{16 \pi^{2} \epsilon}\left[\frac{\lambda}{2}(1+6 \xi)+\frac{1}{3} y_{t}^{2}+\frac{M^{2}}{Z \omega} \frac{\xi}{48}\right. \\
& \left.\quad \times\left(7+120 \xi+144 \xi^{2}\right)-\frac{5 M^{2}}{6 Z} \xi \omega\right] \int \mathrm{d}^{4} x \sqrt{-\bar{g}} \bar{R} \varphi^{2} .
\end{aligned}
$$

Here the first two terms arise due to matter loop while the rest of terms contain quantum gravity corrections. This is in agreement with [26].

\subsection{Effective action and beta functions}

Once we have computed all the relevant graphs at various order of the perturbation theory and their divergent contributions, it is easy to put them together to write the divergent part of effective action and collect all the pieces together. The divergent part of the full effective action is given by

$$
\begin{aligned}
\Gamma_{\text {div }}^{(1)}= & -\frac{1}{16 \pi^{2} \epsilon} \int \mathrm{d}^{4} x\left[\left(\frac{3 M^{2}}{16 Z \omega}+2 y_{t}^{2}\right)(\partial \varphi)^{2}\right. \\
& +\left\{\frac{9 \lambda^{2}}{2}-2 y_{t}^{4}+\frac{5 M^{2}}{8 Z}\left(2 \lambda+\frac{M^{2}}{Z} \xi^{2}\right)\right. \\
& \left.+\frac{M^{2}}{Z \omega} \frac{\lambda}{16}\left(1+48 \xi+144 \xi^{2}\right)+\frac{M^{4}}{8 Z^{2} \omega^{2}} \xi^{2}(1+6 \xi)^{2}\right\} \varphi^{4} \\
& +\left\{\frac{\lambda}{2}(1+6 \xi)+\frac{1}{3} y_{t}^{2}+\frac{M^{2}}{Z \omega} \frac{\xi}{48}\left(7+120 \xi+144 \xi^{2}\right)\right. \\
& \left.-\frac{5 M^{2}}{6 Z} \xi \omega\right\} R \varphi^{2}+\left\{y_{t}^{2}-\frac{25 M^{2}}{8 Z}+\frac{17 M^{2}}{16 Z \omega}\right\} \bar{\theta} i \not \partial \theta \\
& \left.+\left\{2 y_{t}^{3}+\frac{5 M^{2}}{Z} y_{t}-\frac{5 M^{2}}{4 Z \omega} y_{t}\right\} \varphi \bar{\theta} \theta\right] .
\end{aligned}
$$

Once the divergent part of the effective action is written it is easy to compute the beta function of the various couplings by incorporating the effect of the wave-function renormalisation. These are given by

$$
\begin{aligned}
\eta_{\varphi}= & \frac{\mathrm{d} \ln Z_{\varphi}}{\mathrm{d} t}=\frac{1}{16 \pi^{2}}\left[\frac{3 M^{2}}{8 Z \omega}+4 y_{t}^{2}\right] \\
\eta_{\psi}= & \frac{\mathrm{d} \ln Z_{\psi}}{\mathrm{d} t}=\frac{1}{16 \pi^{2}}\left[y_{t}^{2}-\frac{25 M^{2}}{8 Z}+\frac{17 M^{2}}{Z \omega}\right] \\
\frac{\mathrm{d} \lambda}{\mathrm{d} t}= & \frac{1}{16 \pi^{2}}\left[18 \lambda^{2}+8 \lambda y_{t}^{2}-8 y_{t}^{4}+\frac{\lambda M^{2}}{Z}\left(5+\frac{(6 \xi+1)^{2}}{\omega}\right)\right. \\
& \left.+\frac{M^{4} \xi^{2}}{2 Z^{2}}\left(5+\frac{(6 \xi+1)^{2}}{\omega^{2}}\right)\right] \\
\frac{\mathrm{d} \xi}{\mathrm{d} t}= & \frac{1}{16 \pi^{2}}\left[\left(\xi+\frac{1}{6}\right)\left\{4 y_{t}^{2}+6 \lambda+\frac{2 M^{2} \xi}{Z \omega}(3 \xi+2)\right\}\right. \\
& \left.-\frac{5 M^{2}}{3 Z} \xi \omega\right] \\
\frac{\mathrm{d} y_{t}}{\mathrm{~d} t}= & \frac{y_{t}}{16 \pi^{2}}\left[5 y_{t}^{2}+\frac{15 M^{2}}{8 Z}\right]
\end{aligned}
$$

where $t=\ln \left(\mu / \mu_{0}\right)\left(\mu_{0}\right.$ is a reference scale) and $\mathrm{d} / \mathrm{d} t=$ $\mu \mathrm{d} / \mathrm{d} \mu$. Here $Z_{\varphi}$ and $Z_{\psi}$ is the wave-functional renormalisation of scalar and fermion field respectively, while $\eta_{\varphi}$ and $\eta_{\psi}$ is the corresponding anomalous dimension. The beta functions obtained here agree fully with [26,27], while there is partial agreement with [23,82-86]. For completeness we also include the running of the gravitational couplings, which has been taken from past literature $[16,78]$. These are given by 


$$
\begin{aligned}
\frac{\mathrm{d}}{\mathrm{d} t}\left(\frac{Z}{M^{2}}\right) & =-\frac{1}{16 \pi^{2}}\left[\frac{133}{10}+\frac{N_{s}+6 N_{f}}{60}\right] \\
\frac{\mathrm{d}}{\mathrm{d} t}\left(\frac{Z \omega}{M^{2}}\right) & =\frac{1}{16 \pi^{2}}\left[\frac{5}{3} \omega^{2}+5 \omega+\frac{5}{6}+3 N_{s}\left(\xi+\frac{1}{6}\right)^{2}\right] .
\end{aligned}
$$

We will do the RG analysis of the couplings and explore the issue of unitarity later.

\section{Effective potential}

Here we compute the effective potential for the background scalar field $\varphi$, which gets contributions not only from matter fields but also from gravitational sector.

To compute the effective potential for scalar, the background scalar field is taken to be constant. This is sufficient to compute the effective potential. The quantum gravitational fluctuations are considered around a flat background. The fermion fields are likewise decomposed into a constant background (which for simplicity is taken to be zero $\bar{\theta}=\theta=0$ ) plus fluctuations. This simplifies the computation very much. As the ghost action does not depend on the background scalar field $\varphi$, there is no contribution by the ghost to the effective potential, and hence will be ignored in the following. Once the full second variation of the action is performed, we have the hessian needed to compute the one-loop effective potential. This can be obtained directly from Eq. (45) by putting background $\bar{R}=0$ and replacing background covariant derivative with partial derivative. Working with a flat background allows the freedom to work directly in momentum space.

Moreover, in flat space-time the transverse-traceless decomposition of $h_{\mu \nu}$ given in Eq. (42) can be rewritten in an alternative form. In this new decomposition the field components $\sigma$ and $h$ of $h_{\mu \nu}$ are replaced by $s$ and $w$. These new fields $s$ and $w$ are related to the old ones in the following manner:

$s=\frac{h-\square \sigma}{d}, \quad w=\frac{h+(d-1) \square \sigma}{d}$.

The advantage of doing this transformation is to bring out the scalar mode which remains invariant under the diffeomorphism transformation stated in Eq. (8). The field $s$ is therefore gauge invariant, while the field $w$ is longitudinal. So the decomposition of $h_{\mu \nu}$ has two gauge-invariant components, $h_{\mu \nu}^{T T}$ and $s$, with two longitudinal components, $\hat{\xi}_{\mu}$ and $w$. Furthermore, on flat space-time one can also use the set of orthogonal projectors to project $h_{\mu \nu}$ on the various components $h_{\mu \nu}^{T T}, \hat{\xi}_{\mu}, s$ and $w$ (see Appendix B). In terms of the new field variables, the hessian mentioned in Eq. (45) can be rewritten (for $R=0$ ) in a more transparent manner to see clearly the gauge-dependent and gauge-independent part. The hessian for $h_{\mu \nu}^{T T}$ and $\hat{\xi}_{\mu}$ remains the same, while the mix- ing matrix of $\hat{\sigma}, h$ and $\chi$ gets rotated due to the field transformation stated in Eq. (57). The new mixing between the field variables $s, w$ and $\chi$ has a simplified structure. Moreover, this transformation of the field variable is unaccompanied by any non-trivial jacobian in the path integral. The one-loop effective potential is therefore obtained from a simplified hessian,

$$
\begin{aligned}
\Gamma^{(1)} & =\frac{(d+1)(d-2) i}{4} \operatorname{Tr} \ln \left\{-\frac{Z}{M^{2}} \square^{2}-\frac{\xi}{2} \varphi^{2} \square+\frac{\lambda}{4} \varphi^{4}\right\} \\
+ & \frac{(d-1) i}{2} \operatorname{Tr} \ln \left\{\frac{2}{\alpha} \square^{2}+\frac{\lambda}{2} \varphi^{4}\right\} \\
+ & \frac{i}{2} \operatorname{Tr} \ln \left[\operatorname{det}\left(\begin{array}{lll}
S_{s s} & S_{s w} & S_{s \phi} \\
S_{w s} & S_{w w} & S_{w \phi} \\
S_{\phi s} & S_{\phi w} & S_{\phi \phi}
\end{array}\right)\right] \\
- & i \operatorname{Tr} \ln \left(i \gamma^{\mu} \partial_{\mu}-y_{t} \varphi\right),
\end{aligned}
$$

where the entries of the scalar mixing matrix are

$$
\begin{aligned}
& S_{s s}=(d-1)\left[\frac{(d-2) Z \omega}{M^{2}} \square^{2}+\frac{(d-2)}{2 d M^{2}} \xi \varphi^{2} \square-\frac{(d-3) \lambda}{8} \varphi^{4}\right], \\
& S_{s w}=S_{w s}=-\frac{(d-1) \lambda}{8} \varphi^{4}, \\
& S_{s \phi}=S_{\phi s}=-(d-1) \varphi\left[2 \xi \square-\lambda \varphi^{2}\right], \\
& S_{w w}=\frac{2}{\alpha} \square^{2}+\frac{\lambda}{8} \varphi^{4}, \quad S_{w \phi}=S_{\phi w}=-\lambda \varphi^{3}, \\
& S_{\phi \phi}=-2 \square-6 \lambda \varphi^{2} .
\end{aligned}
$$

From the entries of mixing matrix we clearly notice that $S_{S S}$, $S_{s w}, S_{s \phi}$ do not depend on the gauge parameter. The only gauge dependence is in $S_{w w}$.

For a generic case with an arbitrary field variable, the oneloop hessian can be written in the form $\left(-\square-m^{2}\right.$ ) (where $m^{2}$ contains the background field contributions and couplings). In this case the effective potential is given by the general formula

$V_{\mathrm{eff}}^{(1)}=\frac{d_{s}\left(m^{2}\right)^{2}}{64 \pi^{2}}\left(\ln \frac{m^{2}}{\mu^{2}}-\frac{3}{2}\right)$,

where $d_{s}$ is a factor due to the degrees of freedom of the field. The term $3 / 2$ in the bracket can be absorbed by rescaling $\mu^{2}$ as $\bar{\mu}^{2}=\mu^{2} e^{3 / 2}$. By exploiting this generic formula one can write the contribution to the effective potential from the various field modes of the metric fluctuation field, the scalar and the fermion field. In the case for spin-2, the differential operator responsible for the contribution can be factored and has the form $\left(-\square-A_{1} \varphi^{2}\right)\left(-\square-A_{2} \varphi^{2}\right)$ where $A_{1}$ and $A_{2}$ are given by

$$
\begin{aligned}
& A_{1}=-2 \sqrt{\pi}\left[\sqrt{f^{2}\left(4 \pi f^{2} \xi^{2}+\lambda\right)}-2 \sqrt{\pi} f^{2} \xi\right], \\
& A_{2}=2 \sqrt{\pi}\left[\sqrt{f^{2}\left(4 \pi f^{2} \xi^{2}+\lambda\right)}+2 \sqrt{\pi} f^{2} \xi\right],
\end{aligned}
$$

respectively. Here both $A_{1}$ and $A_{2}$ are dimensionless. It should be noted that, for positive $\lambda, A_{1}$ is negative, while $A_{2}$ is positive. If the sign of $\xi$ is reversed, the roles of $A_{1}$ and 
$A_{2}$ get interchanged. A negative $A_{1}$ is tachyonic in nature. This is a source of instability in the effective potential and will give an imaginary contribution to the effective potential. Plugging $A_{1} \varphi^{2}$ and $A_{2} \varphi^{2}$ for the $m^{2}$ in the expression for the effective potential in Eq. (60) and summing the two, we get the contribution of the spin-2 mode to the effective potential.

This imaginary piece, though, is an infrared effect. It is an indication that the background chosen for doing the computation is not stable, and it is a generic feature of gravity coupled with a scalar field in flat space-time at zero temperature $[19,23]$. This is the same as the instability seen in a gas of gravitons at finite temperature, an indication that flat space-time is unstable. This issue has been thoroughly investigated in the past in $[87,88]$. This kind of tachyonic mode will create issues with unitarity, but this one is different from the unitarity issue caused by the ghost of higher-derivative gravity, in the sense that the former is an IR problem and has no effect on the UV physics, while the latter does affect the UV physics also. Since we are interested in sorting out the problem of unitarity caused by ghosts of higher derivatives, we study only this by focussing on the real part of the effective potential, as the imaginary piece is relevant in the IR case and deals with a tachyonic instability only. This is an important realisation as it decouples the two problems: (a) the problem of unitarity caused by higher-derivative ghosts, and (b) the problem of unitarity caused by tachyons. This paper deals with the former problem.

The contribution of the spin- 0 mode is a bit complicated as it involves the scalar mixing matrix. We need to compute the determinant of this mixing matrix and then compute the effective potential of the operator so obtained from this matrix determinant. The operator obtained after the matrix determinant is the following:

$$
\begin{gathered}
-\square^{3}-3 \varphi^{2}\left[\frac{M^{2} \xi}{Z \omega}\left(\xi+\frac{1}{6}\right)+\lambda\right] \square^{2} \\
+\frac{M^{2}}{16 Z \omega} \lambda(24 \xi+1) \varphi^{4} \square-\frac{9 M^{2}}{16 Z \omega} \lambda \varphi^{6} .
\end{gathered}
$$

This operator is a cubic polynomial in $-\square$ and will therefore have three roots. The nature of the roots can be analysed using the discriminant $\Delta$ of the equation formed by putting the cubic polynomial in (62) to zero. We write

$$
\begin{gathered}
d_{0}=1, \quad d_{1}=-3 \varphi^{2}\left[\frac{M^{2} \xi}{Z \omega}\left(\xi+\frac{1}{6}\right)+\lambda\right], \\
d_{2}=-\frac{M^{2}}{16 Z \omega} \lambda(24 \xi+1) \varphi^{4}, \quad d_{3}=-\frac{9 M^{2}}{16 Z \omega} \lambda \varphi^{6}, \\
\Delta=18 d_{0} d_{1} d_{2} d_{3}-4 d_{1}^{3} d_{3}+d_{1}^{2} d_{2}^{2}-4 d_{0} d_{2}^{3}-27 d_{0}^{2} d_{3}^{2} .
\end{gathered}
$$

If $\Delta>0$ then all roots are real, if $\Delta=0$ then there is a multiple root, and $\Delta<0$ then the roots are complex. The operator in Eq. (62) can be factorised as $\left(-\square-B_{1} \varphi^{2}\right)(-\square-$
$\left.B_{2} \varphi^{2}\right)\left(-\square-B_{3} \varphi^{2}\right)$ where $B_{1}, B_{2}$ and $B_{3}$ are dimensionless. As the product of the roots $B_{1} B_{2} B_{3}$ is positive and $B_{1} B_{2}+$ $B_{2} B_{3}+B_{3} B_{1}$ is negative, this will imply that when $\Delta>0$ then two roots will be negative. If $\Delta=0$, then there is a multiple root with negative sign. In the case when $\Delta<$ 0 there is a pair of complex conjugate roots with negative real part and a positive real root. The case with $\Delta \geq 0$ has roots carrying a negative sign, while for $\Delta<0$ the complex conjugate pair has a negative real part. In all these cases the roots can be written as

$B_{1}=a, \quad B_{2}=-r e^{\theta}, \quad B_{3}=-r e^{-\theta}$.

In the case when $\Delta>0, \theta$ is positive and real, in the $\Delta=0$ case $\theta=0$, while in the $\Delta<0$ case $\theta$ is imaginary. The factor of -1 in the parametrisation of the roots can also be exponentiated as $e^{i \pi}$. This factor is the source of tachyonic instability and will give rise to an imaginary contribution in the effective potential. This is similar to the instability caused in the spin-2 case and is an indication that flat space-time is not a true vacuum $[87,88]$. The contribution to the effective potential from the scalar sector is now easily computed using the generalised expression given in Eq. (60). This is done by replacing $m^{2}$ in Eq. (60) with $B_{1} \varphi^{2}, B_{2} \varphi^{2}$ and $B_{3} \varphi^{2}$, and summing all. Using the parametrisation for the roots written in Eq. (64) and employing the properties of exponential functions, one can write the effective potential in simple terms. ${ }^{3}$

The contribution of the fermions needs to be done in a different manner. It arises from $-i \operatorname{Tr} \ln \left(i \gamma^{\mu} \partial_{\mu}-y_{t} \varphi\right)$. This can be written in an alternative form by squaring the operator and by making use of the gamma-matrix properties. This then become $-i / 2 \operatorname{Tr} \ln \left(-\square-y_{t}^{2} \varphi^{2}\right)$. Then using the generalised expression in Eq. (60) one gets the contribution for the fermions. The full effective potential involves the tree-level contributions plus the quantum corrections. The one-loop RG improved full effective action is then given by

$$
\begin{gathered}
V_{\mathrm{eff}}^{(1)}=\frac{\lambda(t)}{4} Z_{\phi}^{4}(t) \varphi^{4}+\frac{Z_{\phi}^{4}(t) \varphi^{4}}{64 \pi^{2}}\left[5 \sum_{i=1}^{2}\left|A_{i}\right|^{2} \ln \frac{\left|A_{i}\right| \varphi^{2}}{\bar{\mu}^{2}}\right. \\
+B_{1}^{2} \ln \frac{B_{1} \varphi^{2}}{\bar{\mu}^{2}}+2 r^{2} \cosh (2 \theta) \ln \frac{r \varphi^{2}}{\bar{\mu}^{2}}+2 r^{2} \theta \sinh (2 \theta) \\
\left.-y_{t}^{4} \ln \frac{y_{t}^{2} \varphi^{2}}{\bar{\mu}^{2}}+\frac{i Z_{\phi}^{4}(t) \varphi^{4}}{64 \pi}\left\{5\left|A_{1}\right|^{2}+2 r^{2} \cosh (2 \theta)\right\}\right],
\end{gathered}
$$

where the $A_{i}, B_{1}, r$ and $\theta$ are dimensionless and the RG time $t$ dependent. When $\theta \rightarrow i \theta, \cosh (2 \theta) \rightarrow \cos (2 \theta)$ and $\sinh (2 \theta) \rightarrow i \sin (2 \theta)$, thereby preventing the switching between real and imaginary parts.

\footnotetext{
3 The discriminant $\Delta$ depends on RG time $t$, and during the RG evolution it can change sign, thereby implying that during the RG evolution $\theta$ can switch from real to imaginary and vice versa.
} 
In the following we will study the real part of the effective potential. We ignore the imaginary part, as the imaginary part arises from the tachyonic modes of the theory and is relevant in the IR case. We are interested in investigating unitarity issues caused by higher-derivatives ghosts. It should be noticed that the effective potential still possesses the $\mathbf{Z}_{2}$ symmetry, as $\varphi^{2}=0$ is an extremum. But due to radiative corrections the real part of the quantum corrected effective potential might develop a vacuum expectation value $(\mathrm{VeV})$ away from zero.

\section{Symmetry breaking}

Due to $\mathrm{RG}$ corrections a $\mathrm{VeV}$ is generated in the effective potential, which then becomes the new vacuum. The original $\varphi^{2}=0$ vacuum becomes unstable under RG corrections and the field migrates to the new vacuum which occur at $\varphi^{2}=\kappa^{2}$. It is given by

$$
\left.\frac{\mathrm{d}}{\mathrm{d} \varphi^{2}} \operatorname{Re}\left(V_{\mathrm{eff}}\right)\right|_{\varphi^{2}=\kappa^{2}}=0 .
$$

At the tree level our original action of the theory is scale invariant and there is no mass parameter to begin with. However, the mass parameter enters the system via RG running, thereby breaking scale invariance. The effective potential so generated not only breaks scale invariance but also breaks the $\mathbf{Z}_{2}$ symmetry. The generation of the $\mathrm{VeV}$ consequently gives mass to the scalar and fermion fields. It also generates an effective Newton constant, beside generating newer interactions. The generated mass and Newton coupling can be expressed in terms of the $\mathrm{VeV} \kappa^{2}$ and all the other couplings as

$m_{s}^{2}=\frac{3}{2} \lambda \kappa^{2}, \quad m_{f}=y_{t} \kappa, \quad G^{-1}=8 \pi \xi \kappa^{2}$.

The generation of mass and Newton's constant makes the propagators for various fields massive. In particular the graviton propagator after the symmetry breaking is the following:

$$
\begin{aligned}
D^{\mu \nu, \alpha \beta}= & 16 \pi G \cdot\left[\frac{\left(2 P_{2}-P_{S}\right)^{\mu \nu, \alpha \beta}}{q^{2}+i \epsilon}+\frac{\left(P_{S}\right)^{\mu \nu, \alpha \beta}}{q^{2}-M^{2} / \omega+i \epsilon}\right. \\
& \left.-\frac{2\left(P_{2}\right)^{\mu \nu, \alpha \beta}}{q^{2}-M^{2}+i \epsilon}\right],
\end{aligned}
$$

where now $G$ is the induced Newton constant and is defined using Eq. (67). The masses $M^{2}$ and $M^{2} / \omega$ are given by

$M^{2}=8 \pi f^{2} \cdot \xi \kappa^{2}, \quad \frac{M^{2}}{\omega}=8 \pi \frac{f^{2}}{\omega} \xi \kappa^{2}$.

The interesting thing about the generation of Newton's constant is that now, as the propagator becomes massive, there is a spin-2 massive ghost that appears in the system, which in the original theory was massless. In the original theory we cannot do the partial-fraction trick in the $h_{\mu \nu}$ propagator, which is possible in the broken phase due to the induced Newton constant $G$. Not only the spin-2 ghost becomes massive but also the scalar mode acquires mass through symmetry breaking. We call this massive scalar mode a 'Riccion'. It should be pointed out that if we had taken $\xi$ to be negative, then there would be tachyons in the broken phase. So the presence of higher-derivatives terms and requiring no tachyons to be generated in broken phase fix the sign of $\xi$. This also generates the right sign for the induced Newton constant. The sign of various couplings in the broken phase is then in accordance with the sign of the parameters taken in $[5,6]$.

At this point we compare the propagator of metric fluctuations written in Eq. (17) with the one written in Eq. (68). The former is before symmetry breaking, while the latter is after symmetry breaking. The former has no mass, while the latter contains masses. Although the appearances of the two are different, one should, however, be careful while counting the propagating degrees of freedom in the two. In the latter case (broken phase) it is easy to count: two massless graviton modes, five massive-tensor ghost modes and one massive scalar mode, thereby making eight propagating degrees of freedom. In the former case (unbroken phase) one should count carefully. Pure $C_{\mu \nu \rho \sigma}^{2}$-gravity has six massless propagating degrees of freedom [89,90]. For pure $R^{2}$ gravity, the theory of two massless propagating degrees of freedom as the linearised field equation $\left(\partial_{\mu} \partial_{\nu}-\eta_{\mu \nu} \square\right) \square h=0$ shows fourth-order time derivatives, thereby totalling the number of degrees of freedom in the unbroken phase to eight. This implies that the numbers of propagating degrees of freedom in both phases are the same, except that in the broken phase some of the modes acquire mass due to symmetry breaking.

The generation of mass for the spin-2 ghost and scalar mode gives us hope to investigate unitarity by using the criterion stated in $[3,5-8]$. In the RG improved effective potential the $\mathrm{VeV}$ has $t$ dependence. This arises because at each energy scale the effective potential has a VeV. This translates into a $t$ dependence of the $\mathrm{VeV}$. The $\mathrm{RG}$ running of the $\mathrm{VeV}$ depends on the running of the other couplings in a complicated manner. This running of the $\mathrm{VeV}$ then translates into running of the generated Newton constant. The running of the $\mathrm{VeV} \kappa^{2}$ can be computed using the expression of the real part of effective potential given in Eq. (65). When $\varphi^{2}=\kappa^{2}$, then we are at the minimum. The minimum condition written in Eq. (66) then translates into the following:

$$
\begin{aligned}
\left.\frac{\mathrm{d}}{\mathrm{d} \varphi^{2}} \operatorname{Re}\left(V_{\mathrm{eff}}\right)\right|_{\varphi^{2}=\kappa^{2}} & =Z_{\phi}^{4}(t) \kappa^{2}\left[\frac{\lambda(t)+\rho_{1}(t)}{2}+\rho_{2}(t)\right. \\
\left.+\rho_{1}(t) \ln \frac{\kappa^{2}(t)}{\bar{\mu}^{2}}\right] & =0
\end{aligned}
$$

where 


$$
\begin{aligned}
\rho_{1}(t)= & \frac{1}{32 \pi^{2}}\left\{5 \sum_{i=1}^{2} A_{i}^{2}+B_{1}^{2}+2 r^{2} \cosh (2 \theta)-y_{t}^{4}\right\} \\
\rho_{2}(t)= & \frac{1}{32 \pi^{2}}\left[5 \sum_{i=1}^{2} A_{i}^{2} \ln \left|A_{i}\right|+B_{1}^{2} \ln B_{1}+2 r^{2} \cosh (2 \theta) \ln r\right. \\
& \left.+2 r^{2} \theta \sinh (2 \theta)-y_{t}^{4} \ln y_{t}^{2}\right]
\end{aligned}
$$

As $\kappa^{2} \neq 0$ and $Z_{\phi} \neq 0$, these overall factors go away and the residual condition simplifies to the expression in the square bracket written in Eq. (70). As $\kappa^{2}(t) / \bar{\mu}^{2}$ is dimensionless, we call it $K(t)$. One can then directly solve for $K(t)$ using Eq. (70) in terms of all couplings of the theory. This also gives the flow of $K$, which is generated due to the flow of various couplings present in the theory. We, however, take a $t$ derivative of the expression in the square bracket of Eq. (70) to compute the beta function of the $K(t)$. This is needed in checking and locating the extrema of $K(t)$. Such extrema are crucial points as will be seen later. We have

$\frac{\mathrm{d} K(t)}{\mathrm{d} t}=-\frac{K(t)}{\rho_{1}(t)}\left[\frac{\lambda^{\prime}(t)+\rho_{1}^{\prime}(t)}{2}+\rho_{2}^{\prime}(t)+\rho_{1}^{\prime}(t) \ln K(t)\right]$.

This is a linear first-order differential equation for the $\ln K(t)$. Plugging the running of various couplings from Eqs. (52)(56) in RHS of Eq. (72) we get the beta function of $K(t)$. This will be a very complicated function of various couplings. Using the running of $K(t)$ we can compute the running of the effective Newton constant by exploiting the expression for the induced $G$ mentioned in Eq. (67). This is given by

$\frac{\mathrm{d} G(t)}{\mathrm{d} t}=-G(t)\left[\frac{1}{\xi(t)} \frac{\mathrm{d} \xi(t)}{\mathrm{d} t}+\frac{1}{K(t)} \frac{\mathrm{d} K(t)}{\mathrm{d} t}+2\right]$.

In order to investigate the issues of unitarity caused by the higher derivative we consider the following combination with $M^{2} / \mu^{2}$. We first note that this is

$\frac{M^{2}}{\mu^{2}}=8 \pi e^{3 / 2} f^{2}(t) \cdot \xi(t) \cdot K(t)$.

Taking the $t$ derivative yields

$\frac{\mathrm{d}}{\mathrm{d} t} \ln \frac{M^{2}}{\mu^{2}}=\frac{1}{f^{2}} \frac{\mathrm{d} f^{2}}{\mathrm{~d} t}+\frac{1}{\xi(t)} \frac{\mathrm{d} \xi(t)}{\mathrm{d} t}+\frac{1}{K(t)} \frac{\mathrm{d} K(t)}{\mathrm{d} t}$.

Similarly the expression for the induced $M^{2} / \omega \mu^{2}$ is

$\frac{M^{2}}{\omega \mu^{2}}=8 \pi e^{3 / 2} \frac{f^{2}(t)}{\omega(t)} \cdot \xi(t) \cdot K(t)$,

and the RG flow of this combination is given by

$$
\begin{aligned}
\frac{\mathrm{d}}{\mathrm{d} t} \ln \frac{M^{2}}{\omega \mu^{2}}= & \frac{1}{f^{2}} \frac{\mathrm{d} f^{2}}{\mathrm{~d} t}-\frac{1}{\omega(t)} \frac{\mathrm{d} \omega(t)}{\mathrm{d} t}+\frac{1}{\xi(t)} \frac{\mathrm{d} \xi(t)}{\mathrm{d} t} \\
& +\frac{1}{K(t)} \frac{\mathrm{d} K(t)}{\mathrm{d} t} .
\end{aligned}
$$

The generation of the $\mathrm{VeV}$ also induces masses for the scalar and the fermion fields, which is mentioned in Eq. (67). Due to the running of the $\mathrm{VeV}$, these masses inherit a running. Then to investigate whether these fields are physically realisable or not, we consider the flow of combinations $m_{s}^{2} / \mu^{2}$ and $m_{f}^{2} / \mu^{2}$. The running of these combinations is given by

$$
\begin{aligned}
\frac{\mathrm{d}}{\mathrm{d} t} \ln \frac{m_{s}^{2}}{\mu^{2}} & =\frac{1}{\lambda(t)} \frac{\mathrm{d} \lambda(t)}{\mathrm{d} t}+\frac{1}{K(t)} \frac{\mathrm{d} K(t)}{\mathrm{d} t}, \\
\frac{\mathrm{d}}{\mathrm{d} t} \ln \frac{m_{f}^{2}}{\mu^{2}} & =\frac{2}{y_{t}(t)} \frac{\mathrm{d} y_{t}(t)}{\mathrm{d} t}+\frac{1}{K(t)} \frac{\mathrm{d} K(t)}{\mathrm{d} t} .
\end{aligned}
$$

\section{Unitarity prescription}

In this section we dictate the algorithm to choose the set of initial conditions for which the theory will have a unitary flow. We start by analysing the RG equations given in Eqs. (52)-(56). The first thing we do is to extract the running $\omega$ using Eqs. (55) and (56). This is given by

$$
\begin{aligned}
\frac{\mathrm{d} \omega(t)}{\mathrm{d} t}= & \frac{f^{2}}{\pi}\left[\frac{5}{3} \omega^{2}+\left\{\frac{183}{10}+\frac{N_{s}+6 N_{f}}{60}\right\} \omega\right. \\
& \left.+\frac{5}{6}+3 N_{s}\left(\xi+\frac{1}{6}\right)^{2}\right] .
\end{aligned}
$$

From this running we notice that as the RHS is always positive, $\omega$ is a monotonically increasing function of $t$. In [5-8] it was shown that in order to avoid tachyonic instability, we should require that $\omega \geq 0$. Here in the present scale-invariant theory we should require the same. This is done in order to prevent the occurrence of tachyons in the broken phase. For every value of $\xi, \omega$ will have two fixed points. We have

$\omega_{1,2}=-\frac{1}{40}\left[221 \mp \sqrt{47961-960 \xi-2880 \xi^{2}}\right]$.

$\omega_{1}$ is repulsive while $\omega_{2}$ is attractive. For $\xi$ small both these fixed points lie in the unphysical tachyonic regime. For large $\xi$ the fixed points are complex conjugate with negative real part. Since $\omega$ is monotonically increasing with $t$, therefore one can alternatively study the RG flows of various parameters in terms of $\omega$. Prevention of tachyonic instability restricts $\omega$ to lie between zero and infinity. This then serves as a good candidate in terms of which the RG flows can be analysed. In [5-8] the RG flows were studied in terms of $\omega$.

The crucial problem in overcoming the issue of unitarity is to choose the right set of initial conditions so that throughout the RG evolution the flow remains unitary in the sense that the ghost mass remains always above the energy scale, and the effective potential does not develop any further instability (other than the ones already present). To prevent the occurrence of this instability requires that the coupling $\lambda$ remains positive throughout the RG flow (as negative $\lambda$ will result in tachyonic instability for scalar field $\phi$ ). This particularly 
depends on the choice of initial condition for Yukawa coupling. If the Yukawa coupling is above a certain threshold then $\lambda$ becomes negative too soon during the RG evolution, making the effective potential unstable. In standard model of particle physics this is an important instability problem where the electroweak vacuum becomes metastable [91] (see references therein). In present case of scale-invariant gravity, we have freedom to explore the set of initial conditions which will give unitary evolution. So we just consider those domains where this instability can be avoided.

In [5-8] it was observed that the RG evolution of $M^{2} / \mu^{2}$ is such that its flow has a unique minimum. This was a crucial feature which allowed us to seek those RG trajectories for which this minimum is above unity. These RG trajectories are the ones for which the flow is unitary (massive tensor mode is not physically realisable). In the present case of gravity being induced from scale-invariant theory we seek a similar behaviour of induced $M^{2} / \mu^{2}$, where now $M^{2}$ is given by Eq. (69), and the flow of $M^{2} / \mu^{2}$ is given in Eq. (75). The flow of $M^{2} / \mu^{2}$ is much complicated in the present case and it is difficult to give a rigorous analytic proof that there exist a minimum in the RG evolution of $M^{2} / \mu^{2}$. From various numerical investigations we realised that a minimum does exist in the evolution of induced $M^{2} / \mu^{2}$. We choose this minimum to be our reference point and choose the initial conditions at this point for all other couplings. The appearance of a minimum in the flow induced $M^{2} / \mu^{2}$ implies that at this minimum the beta function of induced $M^{2} / \mu^{2}$ will vanish,

$$
\left.\frac{1}{f^{2}} \frac{\mathrm{d} f^{2}}{\mathrm{~d} t}\right|_{t=t_{*}}+\left.\frac{1}{\xi(t)} \frac{\mathrm{d} \xi(t)}{\mathrm{d} t}\right|_{t=t_{*}}+\left.\frac{1}{K(t)} \frac{\mathrm{d} K(t)}{\mathrm{d} t}\right|_{t=t_{*}}=0 .
$$

Plugging the RG flows of various coupling in this, will result in a condition satisfied by all the couplings of the theory at this minimum. This will act as a constraint in choosing some of the initial parameters. We first choose the value of $M^{2} / \mu^{2}$ at this point, we call it $\rho_{*}$. We require $\rho_{*}>1$. We have

$$
\left.\frac{M^{2}}{\mu^{2}}\right|_{t=t_{*}}=8 \pi e^{3 / 2} f_{*}^{2} \cdot \xi_{*} \cdot K_{*}=\rho_{*}>1,
$$

where $f_{*}^{2}, \xi_{*}$ and $K_{*}$ are initial values of $f^{2}, \xi$ and $K$, respectively. The imposition of this constraint makes sure that the mass of the spin-2 ghost mode remains above the running energy scale. This will imply that one of the three unknowns $f_{*}^{2}, \xi_{*}$ and $K_{*}$ can be expressed in terms of other two. We choose to write $K_{*}$ in terms of $f_{*}^{2}$ and $\xi_{*}$. At this point we also choose $f_{*}^{2} \ll 1$. Now the left unknowns are $\lambda_{*},\left(y_{t}\right)_{*}, \omega_{*}$ and $\xi_{*}$. In order to choose the matter couplings $\lambda_{*}$ we use our knowledge of non-gravitational system. In such system the running $\lambda$ always hits the Landau pole if the initial value of Yukawa coupling is below certain threshold, beyond which $\lambda$ becomes negative leading to instability. We accordingly choose $\lambda_{*} \lesssim 0.1$.

At this point we analyse the beta function for the coupling $\xi$. In this theory we have the freedom to choose $\xi$ to be very large $(\gtrsim 10)$. This is primarily because in the perturbation theory the coupling strength of vertex containing $n$-gravitons and two scalars is $\sim \xi\left(\sqrt{f^{2}}\right)^{n}$ and $\sim \xi\left(\sqrt{f^{2} / \omega}\right)^{n}$. Since $f^{2} \ll 1$, so this give us freedom to choose $\xi$ to be very large while still being in the realm of perturbation theory. ${ }^{4}$ For $\xi$ large the beta functions of various coupling acquires a simplified form. Although the beta functions become simplified but still they are complicated enough to require the analysis to be done numerically. We tend to explore numerically this regime of the parameters.

We choose to work in regime where $-\Delta / \varphi^{6}=\epsilon \ll 1$, where $\Delta$ is the discriminant mentioned in Eq. (63). In this regime there will one positive root for $-\square$ and a complex conjugate pair with negative real part. Under the RG flow $\Delta / \varphi^{6}$ will also run. We choose the initial parameters in such a way so that at the initial point $\epsilon \ll 1$. By reversing this argument we say that we start with $\epsilon \ll 1$ and solve for the initial parameters under this constraint. This fixes the initial value problem completely. With the chosen $f_{*}^{2} \ll 1$ $\left(\lesssim 10^{-6}\right), \rho_{*}>1, \lambda_{*} \lesssim 0.1$ and $\xi_{*} \gg 1\left(\gtrsim 10^{2}\right)$, we use the constraint dictated by $\epsilon \ll 1$ to solve for $\omega_{*}$. From the four different solution for $\omega_{*}$, we choose the one which real and positive (to avoid tachyons). ${ }^{5}$ Knowledge of $\xi_{*}$ gives the initial value of $K_{*}$ by using the relation given in Eq. (82). We then plug these into the minimum constraint given in Eq. (81). This constraint contains the Yukawa coupling in quadratic form, and therefore on solving gives two equal and opposite values for $y_{t *}$. One can choose either of the sign of Yukawa coupling for the initial condition. The flow of all the other couplings does not depend on this sign. Once the initial parameters are known we can solve the RG flows and compute the flow of induced $M^{2} / \mu^{2}$ to see if it remains above unity throughout the $\mathrm{RG}$ evolution.

\section{Numerical analysis}

We tried several possible values of various parameters in order to see how the flows are for various initial conditions, and did the analysis case by case systematically.

\footnotetext{
${ }^{4}$ In the case on Einstein-Hilbert gravity with only Newton's constant $G$, the coupling strength is $\xi(\sqrt{G})^{n}$ for a vertex containing $n$ gravitons and two scalars.

${ }^{5}$ It is noticed that if $\epsilon<0$, then all solutions for $\omega_{*}$ will be negative and will lie in unphysical tachyonic regime. This knowledge also requires one to take $\epsilon>0$.
} 
Table 1 Initial values for the various coupling parameters. These are for $f_{*}^{2}=10^{-6}, 10^{-7}$ and $10^{-8}$. We took $\lambda_{*}=0.1$ and $\rho_{*}=1.5$. Here for three different values of $f_{*}^{2}$ we took $\epsilon=10^{-12}, 10^{-15}$ and $10^{-16}$.
As $\xi_{*}$ increase, the value of $\omega_{*}$ and $y_{t *}$ increase. The number of e-folds tend to decrease as $\xi_{*}$ increases. For smaller $\xi_{*}$ the reason we see less e-folds is due to numerical precision of machine

\begin{tabular}{|c|c|c|c|c|c|c|c|c|c|c|c|}
\hline \multicolumn{4}{|c|}{$\overline{f_{*}^{2}}=10^{-6}$} & \multicolumn{4}{|c|}{$f_{*}^{2}=10^{-7}$} & \multicolumn{4}{|c|}{$f_{*}^{2}=10^{-8}$} \\
\hline$\xi_{*}$ & $\omega_{*} \times 10^{-4}$ & $y_{t *}$ & $T_{r}$ & $\xi_{*}$ & $\omega_{*} \times 10^{-6}$ & $y_{t *}$ & $T_{r}$ & $\xi_{*}$ & $\omega_{*} \times 10^{-6}$ & $y_{t *}$ & $T_{r}$ \\
\hline 1 & 3.0536 & 0.4522 & $\sim 215$ & $10^{2}$ & 3.0536 & 0.4536 & $\gtrsim 235$ & $10^{2}$ & 3.0536 & 0.4522 & $\gtrsim 230$ \\
\hline 10 & 3.0536 & 0.4532 & $\sim 215$ & $5 \times 10^{2}$ & 3.0536 & 0.4543 & $\gtrsim 267$ & $10^{3}$ & 3.0536 & 0.4532 & $\gtrsim 212$ \\
\hline $10^{2}$ & 3.0549 & 0.4543 & $\gtrsim 217$ & $10^{3}$ & 3.0537 & 0.4546 & $\sim 437$ & $10^{4}$ & 3.0549 & 0.4542 & $\gtrsim 235$ \\
\hline $5 \times 10^{2}$ & 3.0869 & 0.4562 & $\gtrsim 305$ & $2 \times 10^{3}$ & 3.0542 & 0.4551 & $\sim 250$ & $5 \times 10^{4}$ & 3.0869 & 0.4562 & $\gtrsim 305$ \\
\hline $10^{3}$ & 3.1840 & 0.4603 & $\sim 356$ & $5 \times 10^{3}$ & 3.0569 & 0.4561 & $\sim 120$ & $10^{5}$ & 3.1840 & 0.4603 & $\sim 356$ \\
\hline $5 \times 10^{3}$ & 5.3737 & 0.5355 & $\sim 60$ & $10^{4}$ & 3.0669 & 0.4587 & $\sim 72$ & $5 \times 10^{5}$ & 5.3736 & 0.5355 & $\sim 58$ \\
\hline
\end{tabular}

\subsection{Fixed $\lambda_{*}$}

We first considered case with fixed $\lambda_{*}$, while we took different values for $f_{*}^{2}$, and for each $f_{*}^{2}$ we explored a range of $\xi_{*}$. Throughout we took $\rho_{*}=1.5$ (there is nothing special about this number, as long as long as $\left.\rho_{*}>1\right)$. We considered the three different values for $f_{*}^{2}=10^{-6}, 10^{-7}$ and $10^{-8}$. We have freedom over the choice of $-\Delta_{*} / \varphi^{6}=\epsilon$. It is seen that with $f_{*}^{2}$ fixed, when $\epsilon$ is made smaller, then $\omega_{*}$ increases. However, the $y_{t *}$ obtained first decreases to a minimum before rising again and becoming stable. We choose $\epsilon$ near this minimum, so that we have a higher number of e-folds in the RG flows. It turns out that for each value of $f_{*}^{2}$ the position of the occurrence of this minimum will be different. For smaller $f_{*}^{2}$, the minimum occurs at a smaller value of $\epsilon$. Thus for $f_{*}^{2}=10^{-6}, 10^{-7}$ and $10^{-8}$, the minimum for $\epsilon$ occurs around $10^{-12}, 10^{-15}$, and $10^{-16}$, respectively. We consider these cases in succession.

The number of e-folds from the Planck time to current galactic scale is $\sim 130$. This stands then as another guiding principle to choose set of the initial parameters. It is noticed that when $f_{*}^{2}$ is made smaller than the allowed upper value of $\xi_{*}$ (which is chosen so that we have $\gtrsim 100$ e-folds) increases. This can be understood by considering the strength of vertices. For vertex containing one graviton leg and two scalar leg, the interaction strength $\sim \xi \sqrt{f^{2}}$. Demanding perturbation theory to remain valid implies $\xi_{*} \sqrt{f_{*}^{2}} \lesssim 1$, which explains the behaviour. We keep $\xi_{*}$ large so that there is sufficient communication between the matter and gravity sector. In Table 1 we tabulate our findings for $f_{*}^{2}=10^{-6}, 10^{-7}$ and $10^{-8}$.

We then plot the flows of various parameters for the choice of initial parameters written in Table 1. Each flow is interesting to analyse. The flow of the coupling $\xi$ for various choices of the initial conditions is shown in Fig. 5. The plot shown in Fig. 5 is for $f_{*}^{2}=10^{-8}$ only, for other values we observe a similar qualitative behaviour, which will not be shown here. From the running of $\xi$ shown in Fig. 5 we notice that the

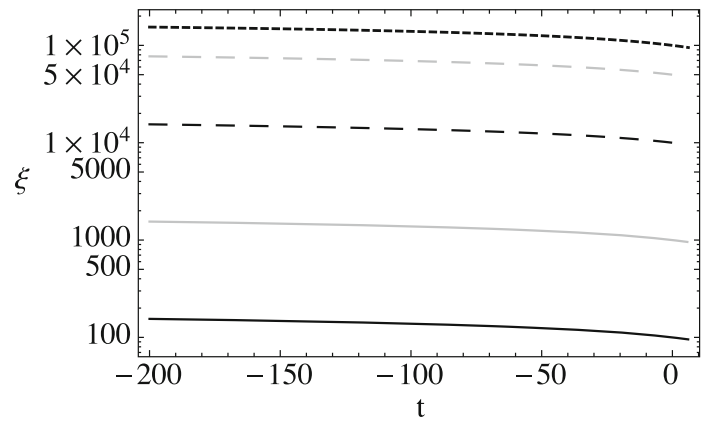

Fig. 5 Running of coupling $\xi$ for various values of initial conditions $\xi_{*}=10^{2}$ (black solid line), $10^{3}$ (grey solid line), $10^{4}$ (big dashed black line), $5 \times 10^{4}$ (big dashed grey line) and $10^{5}$ (small dashed black line). For these flows we took $f_{*}^{2}=10^{-8}, \lambda_{*}=0.1, \rho_{*}=1.5$ and $\epsilon=10^{-16}$

parameter $\xi$ runs to smaller values in the UV regime. This might be an indication of the possible existence of an UV stable fixed point, but it is hard to give a robust answer in this paper. This, however, can be justified by looking at the beta function of $\xi$ given in Eq. (53) whose r.h.s. can be seen to vanish for a certain choice of coupling parameters.

The flow of the matter couplings $\lambda$ and $y_{t}$ for various initial conditions is shown on the left and the right of Fig. 6, respectively. For smaller values of $\xi_{*}$ the flow of these couplings remains almost the same, while deviations are seen for large $\xi_{*}$. This is again plotted for $f_{*}^{2}=10^{-8}$, while for other values of $f_{*}^{2}$ a qualitatively similar behaviour is seen. In the UV case the flow of $\lambda$ is seen to bend and run toward smaller values, which is caused by the Yukawa coupling.

The flow of the VeV induces a flow in the Newton constant. The flow of the induced Newton constant for various initial conditions is shown in Fig. 7. The induced Newton constant goes to zero in the UV and in the IR cases. In UV it is seen to go to zero at a finite energy scale. This is similar to the flow of Newton's constant observed in [5-8], where the original action was not scale invariant and contained Einstein-Hilbert piece in the higher-derivative action. This is rather interesting 


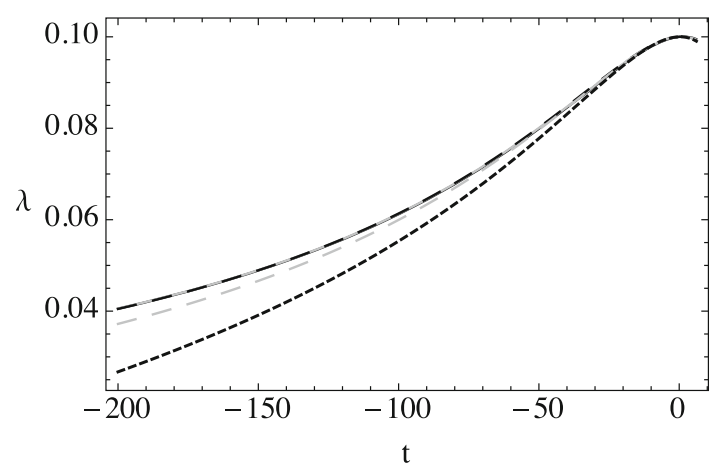

Fig. 6 Running for matter couplings $\lambda$ and $y_{t}$ in left and right, respectively. These are plotted for $f_{*}^{2}=10^{-8}, \lambda_{*}=0.1, \rho_{*}=1.5$ and $\epsilon=10^{-16}$. We considered the following initial conditions for $\xi_{*}=10^{2}$

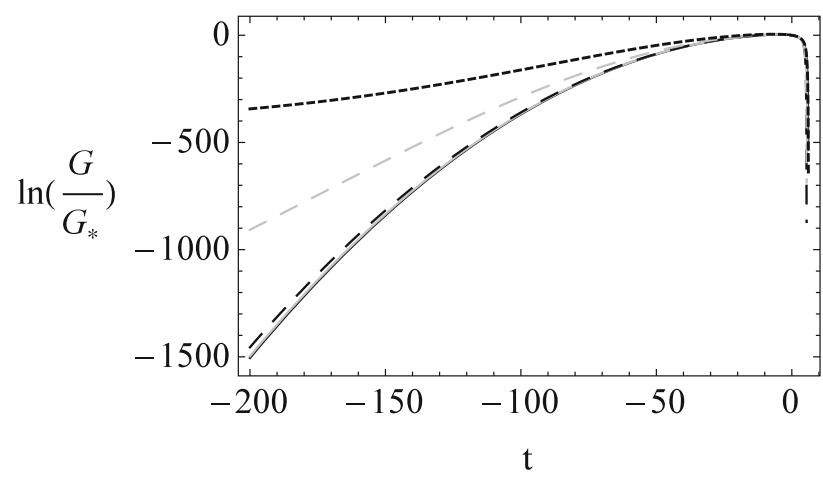

Fig. 7 The flow of the induced Newton constant. Here we plot $\ln \left(G / G_{*}\right)$ for the case $f_{*}^{2}=10^{-8}, \lambda_{*}=0.1, \rho_{*}=1.5$ and $\epsilon=10^{-16}$. The flow is computed for five different values of $\xi_{*}=10^{2}$ (black solid line), $10^{3}$ (grey solid line), $10^{4}$ (big dashed black line), $5 \times 10^{4}$ (big dashed grey line) and $10^{5}$ (small dashed black line). Both in UV and IR the flow goes very close to zero

to note. Again this is just a numerical observation and not a rigorous analytic argument. By varying the value of $f_{*}^{2}$ we notice that the qualitative features of the graph remain the same.

Figure 8 shows the flow of the parameters $M^{2} / \mu^{2}$ and $M^{2} / \omega \mu^{2}$ in left and right, respectively. The flow of $M^{2} / \mu^{2}$ is such that it is always above unity $\left(M^{2} / \mu^{2}>1\right)$. This means that the propagator of metric fluctuation after symmetry breaking does not witness the ghost pole, as the problematic ghost mode is never realised and never goes on-shell. We observe this to happen for a large domain of coupling parameter space. A similar running of the parameter $M^{2} / \mu^{2}$ was also observed in [5-8], and it was used to establish a unitarity criterion for the higher-derivative gravity. The flow of the parameter $M^{2} / \omega \mu^{2}$ is different from the one observed in [5-8], where a monotonic behaviour was seen. In the present case we see a convex structure with a single minimum in the flow. If we require $M^{2} /\left.\mu^{2}\right|_{*}>1$, then it does not imply

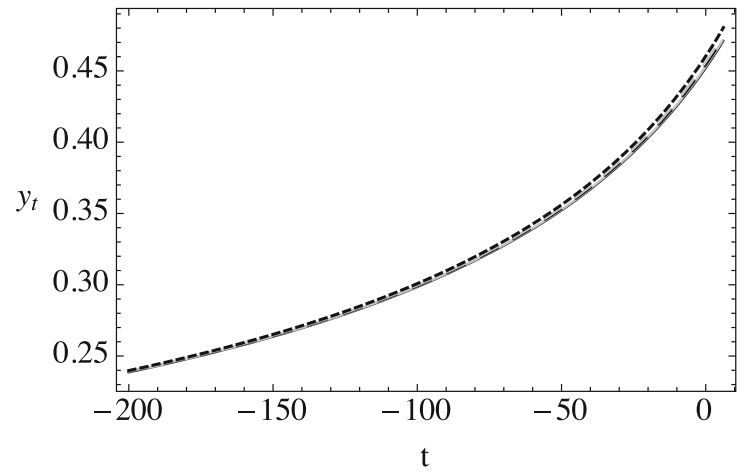

(black solid line), $10^{3}$ (grey solid line), $10^{4}$ (big dashed black line), $5 \times 10^{4}$ (big dashed grey line) and $10^{5}$ (small dashed black line)

that $M^{2} /\left.\omega \mu^{2}\right|_{*}>1$. However, the reverse is always true i.e. $M^{2} /\left.\omega \mu^{2}\right|_{*}>1$ implies $M^{2} /\left.\mu^{2}\right|_{*}>1$. By choosing $\rho_{*}$ to be large enough one can make the scalar mode also physically unrealisable.

We then consider the induced masses in the matter sector and consider the flow of combinations $m_{s}^{2} / \mu^{2}$ and $m_{f}^{2} / \mu^{2}$, where $m_{s}$ and $m_{f}$ is given in Eq. (67). The induced RG running of them is shown in Fig. 9. The running of these is interesting in the sense that both of them have a minimum. The value at the minimum depends crucially on the initial parameters chosen so as to make the flow unitary. If we choose $\rho_{*}$ large enough then it is possible that the flows of $m_{s}^{2} / \mu^{2}$ and $m_{f}^{2} / \mu^{2}$ will be such that the scalar and fermion will never be realised during the whole RG flow, and they never go onshell. In that sense they affect the theory indirectly and only gravitationally_but they never go on-shell.

\subsection{Fixed $f_{*}^{2}$}

In the previous subsection the case with fixed $\lambda_{*}$ was investigated. It is worth checking the robustness of the qualitative features when other parameters are varied. One particular important parameter is the $\lambda_{*}$. It is important to see how the situation changes when $\lambda_{*}$ is increased. For this we fix the values of $f_{*}^{2}=10^{-8}, \xi_{*}=10^{5} \rho_{*}=1.5$. We took a range of values of $\lambda_{*}=0.2,0.25,0.3,0.35$ and 0.45 . Although the qualitative features are the same, there are minor differences. In each case we always witness that the running $M^{2} / \mu^{2}$ has a minimum, and the flow is always above unity. This further establishes that there always exist a minimum in the flow of induced $M^{2} / \mu^{2}$, and it also implies that by choosing right set of initial condition it is possible to make the massive tensor ghost innocuous.

As the system contain a mixture of several couplings, which are all evolving in different manner, the dynamics of system is rich and interesting. This becomes more apparent 


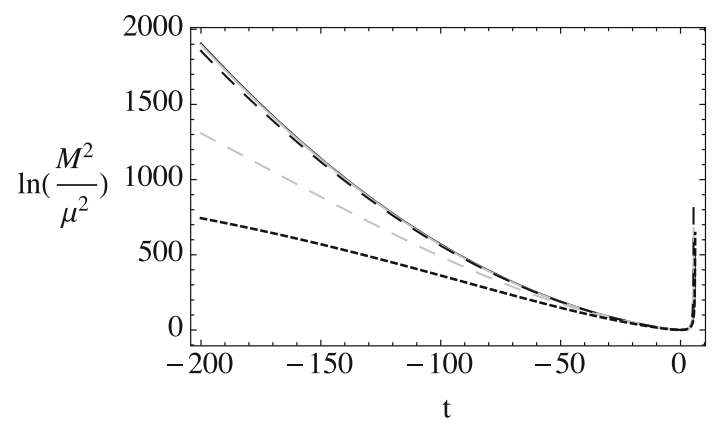

Fig. 8 The running of induced $\ln \left(M^{2} / \mu^{2}\right)$ and $\ln \left(M^{2} / \omega \mu^{2}\right)$ for the case $f_{*}^{2}=10^{-8}, \lambda_{*}=0.1, \rho_{*}=1.5$ and $\epsilon=10^{-16}$. The flow is computed for five different values of $\xi_{*}=10^{2}$ (black solid line), $10^{3}$ (grey solid line), $10^{4}$ (big dashed black line), $5 \times 10^{4}$ (big dashed grey line)

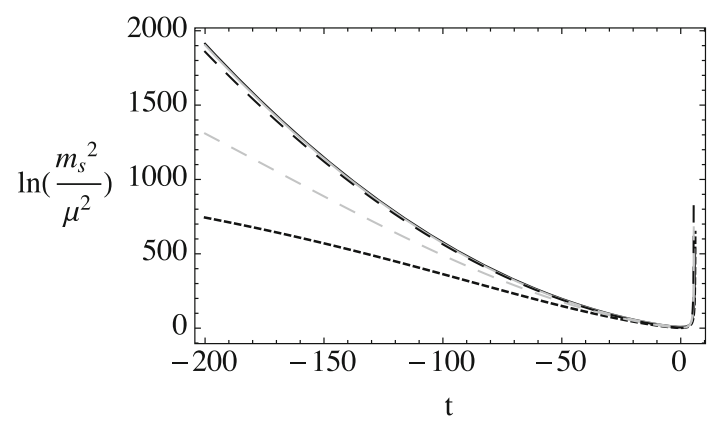

Fig. 9 The running of induced $\ln \left(m_{s}^{2} / \mu^{2}\right)$ and $\ln \left(m_{f}^{2} / \mu^{2}\right)$ for the case $f_{*}^{2}=10^{-8}, \lambda_{*}=0.1, \rho_{*}=1.5$ and $\epsilon=10^{-16}$. The flow is computed for five different values of $\xi_{*}=10^{2}$ (black solid line), $10^{3}$ (grey solid line), $10^{4}$ (big dashed black line), $5 \times 10^{4}$ (big dashed grey line) and $10^{5}$

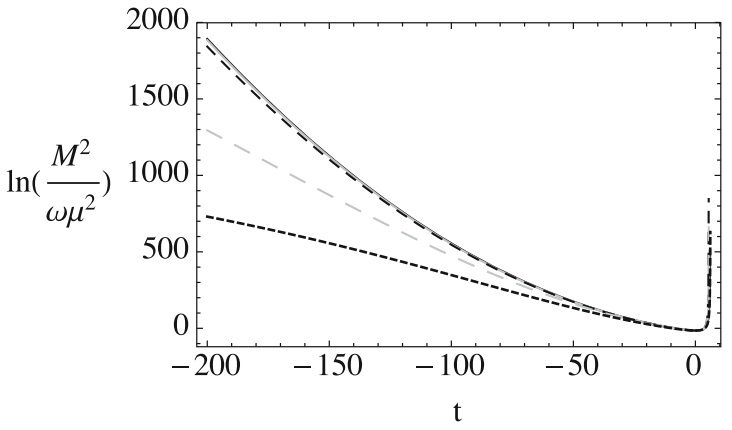

and $10^{5}$ (small dashed black line). In all cases it is seen that the flow has a minimum. If the flow is such that we have $M^{2} / \mu^{2}>1$ throughout the flow, then this spin-2 ghost mode never goes on-shell, and the theory satisfies unitarity

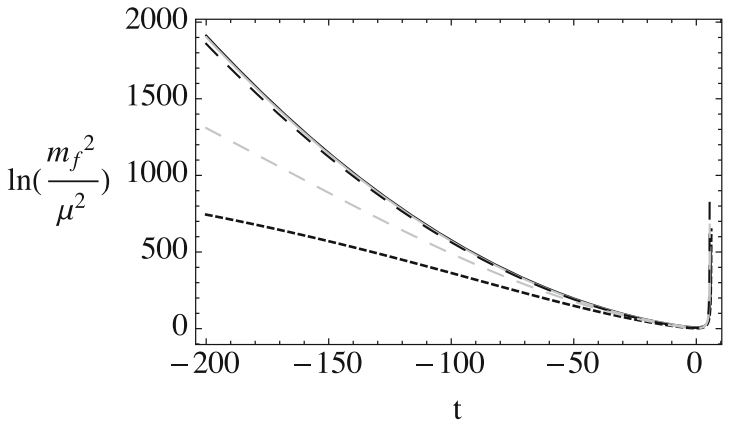

(small dashed black line). In all cases it is seen that the flow has a minimum. If throughout the flow both $m_{s}^{2} / \mu^{2}>1$ and $m_{f}^{2} / \mu^{2}>1$, then both of them never go on-shell
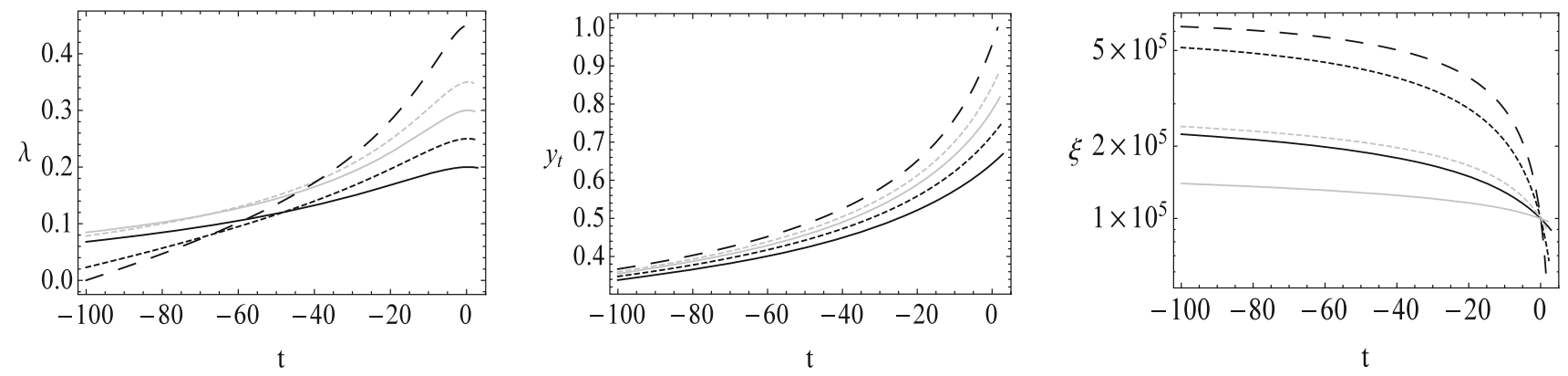

Fig. 10 Running for couplings $\lambda, y_{t}$ and $\xi$ in left, centre and right, respectively. These are plotted for $f_{*}^{2}=10^{-8}, \lambda_{*}=0.2$ (black solid line), 0.25 (black short-dashed line), 0.3 (grey solid line), 0.35 (grey short-dashed line) and 0.45 (black long-dashed line). We took $\rho_{*}=1.5$ and $\xi_{*}=10^{5}$

when we plot the running of various parameters. The flow of $\lambda, y_{t}$ and $\xi$ is shown in Fig. 10. These flows are very much similar to the ones shown for fixed $\lambda_{*}$ in the previous sub-section. It is seen that as $\lambda_{*}$ is increased the flow of $\xi$ decrease more sharply in the UV case, and in the IR case the flow goes to higher values, even though starting point is the same. The running of Yukawa coupling is simple, in the sense that when $\lambda_{*}$ increases, so does $y_{t *}$ and accordingly the whole RG trajectory for Yukawa coupling. The flow of $\lambda$ is interesting. For higher $\lambda_{*}, \xi$ flows to higher values in the IR. This makes the $\lambda$ to run faster toward zero in the IR. In the UV the RG trajectories for $\lambda$ has self-similarity.

The flow of $G, M^{2} / \mu^{2}$ and $M^{2} / \omega \mu^{2}$ is shown in left, centre and right respectively in Fig. 11. The qualitative behaviour is the same in the sense that the induced Newton constant goes to zero in the UV at a finite energy scale. It goes to zero in the IR. The RG flows for $M^{2} / \mu^{2}$ and $M^{2} / \omega \mu^{2}$ have same qualitative features, and tensor ghost is physically unreal- 


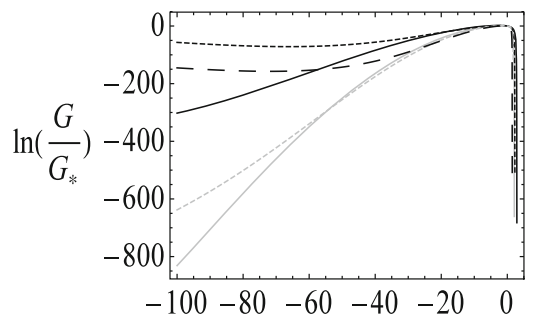

$\mathrm{t}$

Fig. 11 The running of induced $\ln \left(G / G_{*}\right), \ln \left(M^{2} / \mu^{2}\right)$ and $\ln \left(M^{2} / \omega \mu^{2}\right)$ in left, centre and right respectively. These are plotted for $f_{*}^{2}=10^{-8}, \lambda_{*}=0.2$ (black solid line), 0.25 (black short-dashed

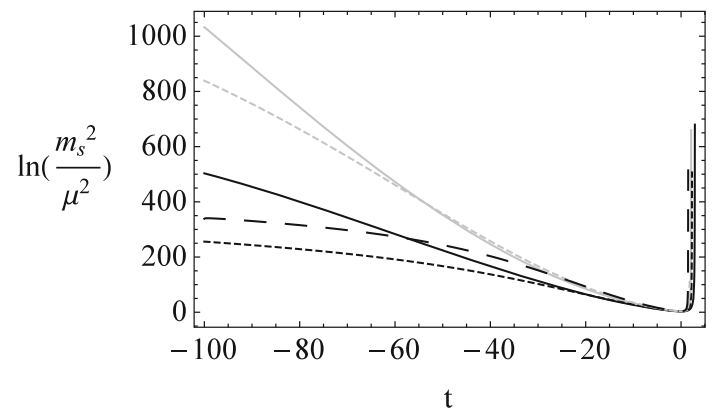

Fig. 12 The running of induced $\ln \left(m_{s}^{2} / \mu^{2}\right)$ and $\ln \left(m_{f}^{2} / \mu^{2}\right)$ in the left and right, respectively. These are plotted for $f_{*}^{2}=10^{-8}, \lambda_{*}=0.2$ (black solid line), 0.25 (black short-dashed line), 0.3 (grey solid line),

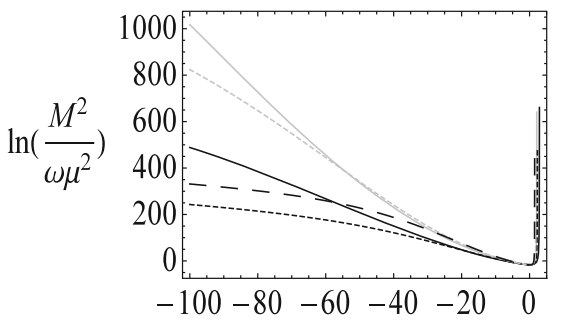

$\mathrm{t}$

line), 0.3 (grey solid line), 0.35 (grey short-dashed line) and 0.45 (black long-dashed line). We took $\rho_{*}=1.5$ and $\xi_{*}=10^{5}$

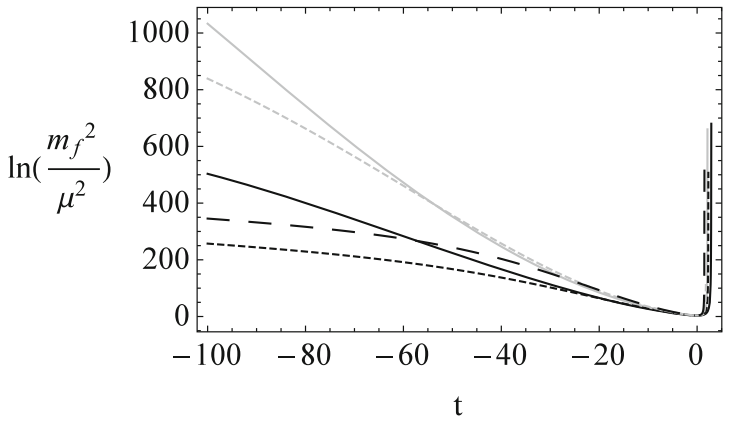

0.35 (grey short-dashed line) and 0.45 (black long-dashed line). We took $\rho_{*}=1.5$ and $\xi_{*}=10^{5}$. Choosing appropriate $\rho_{*}$ it is possible to make both the scalar and the fermion unrealisable

Fig. 13 The running of various dimensionless couplings. For this we took $f_{*}^{2}=10^{-8}$, $\lambda_{*}=0.1, \xi_{*}=10^{5}$. We took $\rho_{*}=1.5$ and $\epsilon=10^{-16}$, which gave $y_{t *}=0.46$ and running energy scale $\omega_{*}=3.18 \times 10^{6}$. Here $\mu$ is the

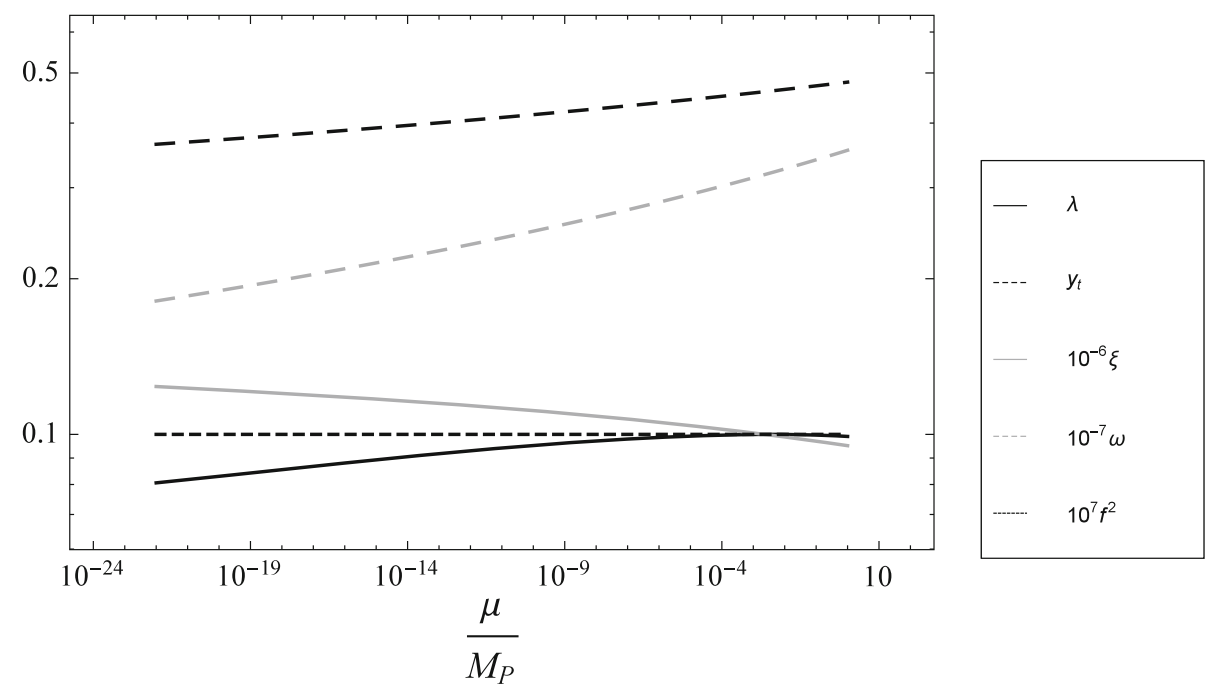

isable even when we increase $\lambda_{*}$. Choosing appropriate $\rho_{*}$ will make the Riccion also physically unrealisable. The RG flow of Riccion mass is different from the one seen in [5-8]. The flow of induced masses in the matter sector has similar qualitative features and is shown in Fig. 12.

\subsection{Fixing Planck's scale}

The renormalisation group invariance ensures that the flow of couplings does not depend on the reference point $\mu_{0}$. This 
Fig. 14 The running of various induced masses and the induced Newton constant. For this we took $f_{*}^{2}=10^{-8}, \lambda_{*}=0.1$, $\xi_{*}=10^{5}$. We took $\rho_{*}=1.5$ and $\epsilon=10^{-16}$, which gave

$y_{t *}=0.46$ and

$\omega_{*}=3.18 \times 10^{6}$. Here $\mu$ is the running energy scale

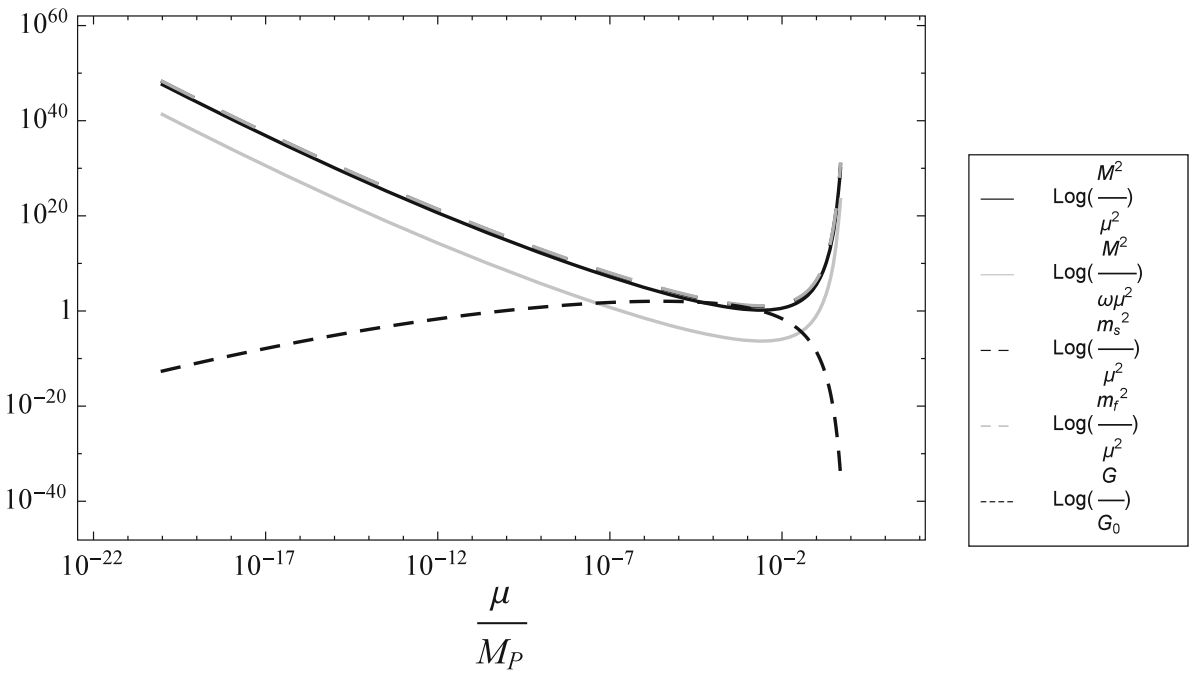

gave us freedom to choose the reference point without any conditions. As a result for the sake of convenience we choose it to be the point where the flow of $M^{2} / \mu^{2}$ has a minimum, where the initial conditions for the flow are imposed. However, an interesting thing to ask is to how to relate it with the phenomenology? In the sense how does the running of various parameter look like when compared to Planck's scale $M_{P l}$, whose value is around $1.22 \times 10^{19} \mathrm{GeV}$ ? This is interesting point to ponder on. For this we study the running of induced $G$, which runs strongly in the UV and goes to zero. From observations of astrophysics and cosmology we know $G_{\text {Newton }}$ remains constant for a large energy range. However, it is usually expected that it will undergo strong running near the Planck scale. For this reason we choose $\mathrm{MPl}_{\mathrm{Pl}}$ in the regime where induced $G$ witnesses a strong running i.e. near the point where induced $G$ goes to zero. Once this is fixed one can plot the flow of various coupling parameters and induced masses. These are presented in Figs. 13 and 14.

\section{Conclusion}

Here in this paper the idea of gravity being induced from scale-invariant theory is considered. The fundamental theory is a coupled system of scale-invariant matter and higherderivative gravity. The Lorentzian path integral of this fundamental theory incorporates quantum fluctuations from both matter and gravity. The matter sector is taken to be simple (a scalar and a Dirac fermion).

The effective action of the theory is computed in the $4-\epsilon$ dimensional regularisation procedure. The divergent part of which gives the RG flow of the various coupling parameters of the theory. These have been computed in past also. We did it again in order to verify the past results. We agree fully with the past literature $[26,27]$. We then compute the oneloop RG improved effective potential of the scalar field on the flat space-time. This gets contributions from both the grav- itational and the matter degrees of freedom. This effective potential, however, contains an instability which comes up as an imaginary piece in the effective potential. The straightforward interpretation of the appearance of an imaginary piece in the effective potential is an indication that the background (flat space-time and constant scalar background) is not stable, and it will decay.

The reason for the occurrence of this instability is probed. It is found that this is purely gravitational in nature, in the sense that it arose from occurrence of tachyonic modes in the spin-2 gravitational sector and the gravitational scalar sector. These kind of tachyonic instabilities have been investigated in past $[87,88]$, and is a characteristic feature of gravitational theories coupled with scalar in flat space-time. At finite temperature this instability (also known as Jeans instability) results in collapse of gas of gravitons. While the occurrence of this instability is a disturbing feature of the theory and is unavoidable, it is, however, an IR problem and has no effect on the UV physics. In this paper we considered a different feature of theory. We investigate the issue of ghost appearing due to the presence of higher-derivative terms in the theory, which affect also the UV physics. This is done by investigating only the real part of the effective potential and ignoring the instability caused by the tachyonic modes.

The real part of effective potential develops a VeV. This breaks the scale symmetry and induces mass scale, which in turn generates Newton's constant and masses for matter fields. The propagator of the metric fluctuation field now has mass, and in the broken phase it is easy to see problematic massive tensor ghost of the theory which remains shrouded in the symmetric phase. The scalar mode (Riccion) of the metric fluctuation also picks a mass in the broken phase. The $\mathrm{VeV}$ has a running, which in turn induces a running in the various parameters that are generated in the broken phase. It therefore seems sensible to ask question on behaviour of massive tensor ghost under this running in the broken phase, which is the most important aim of the paper. 
The induced running in the various parameters generated in the broken phase allows to investigate the running of $M^{2} / \mu^{2}$ (where $M$ is the induced mass of tensor ghost). The crucial task of the paper was to see whether there exists a domain of coupling parameters space where it is possible to make $M^{2} / \mu^{2}>1$ throughout the whole RG trajectory. Satisfactory arrangement of $M^{2} / \mu^{2}>1$ will imply that the massive tensor ghost is never physically realised and never goes on-shell. This issue is, however, studied numerically, as the complexity of the beta functions and the complicated running of the induced parameters in broken phase hinders to make analytic progress.

The last part of paper is devoted to numerically studying this issue. A prescription to choose the set of initial conditions so that $M^{2} / \mu^{2}>1$ for the whole RG trajectory is stated. This involves solving certain constraints. It is realised that the flow of $M^{2} / \mu^{2}$ has a unique minimum at a certain point along the RG trajectory. The existence of such a point was analytically proved in the context of higher-derivative gravity including Einstein-Hilbert term [5,6], as the RG equation were simpler. In the present paper, however, it is not possible to achieve this analytically and numerical support was taken to get evidence for the existence of such a minimum. We do see that for a large domain of parameter space such a unique minimum does exist, and requiring that $M^{2} / \mu^{2}>1$ at this minimum implies that $M^{2} / \mu^{2}>1$ for the whole RG trajectory. This, though, is not a robust analytic proof but stands as a strong evidence. We considered a different set of initial conditions by varying various parameters in a systematic fashion. In each case it was seen that the minimum in the flow of $M^{2} / \mu^{2}$ always exists and the unitarity criterion can be met.

In this domain of coupling parameter space, where $M^{2} / \mu^{2}>1$ for the whole RG trajectory, the behaviour of the other parameters is studied. The first important thing that is to be noticed is the existence of a finite UV cutoff in the theory, which was also noticed in [5-8]. Even though we do the analysis in dimensional regularisation, still a cutoff emerges dynamically from the RG equations. Beyond this point the flow cannot be continued and knowledge of higher-loop contributions is needed. In [5-8] we showed analytically that at this point the coupling $\omega$ diverges. In the present context we noticed this numerically. The behaviour of the matter coupling $\lambda$ has an interesting flow. It is seen to increase monotonically, but in the UV case this stops and starts to decrease. This is due to Yukawa coupling. The flow of the Yukawa coupling $y_{t}$ increases monotonically, and it stops when the cutoff is reached. In the case of $R \varphi^{2}$ coupling, the coupling starts to run only near the UV, where it is seen to go toward smaller values, hinting that there might perhaps exist a stable fixed point.

The flow of the induced Newton constant $G$ is interesting. It approaches zero both in UV and IR. In UV it vanishes at finite energy scale. This was something which was also observed in [5-8], where Einstein-Hilbert was present in the bare action of the theory and was not induced. In that respect this is surprising that in the present picture of the EinsteinHilbert term being induced from scale-invariant theory, the flow of the induced gravitational coupling is qualitatively similar to the case where EH term is present in the theory to begin with. Such vanishing of induced $G$ is although unexpected but a welcome feature. This is opposite to the widely known feature in Einstein-Hilbert gravity (without higher derivatives) where Newton's constant becomes very large in the UV case. However, those results cannot be trusted as they appear in non-renormalisable theories. In the presence of higher derivatives the situation changes in UV. Such a vanishing of Newton's constant means that, in the UV case, gravity decouples from matter, although gravitational selfinteractions continue to exist. Such a behaviour will have consequences in the early universe, and it also justifies the use of a flat background in the UV case. This softening can also be used in addressing the Higgs naturalness problem [27].

The flow of combination $M^{2} / \omega \mu^{2}$ is, however, a bit different from what has been witnessed in [5-8], in the sense that the function $M^{2} / \omega \mu^{2}$ is no longer a monotonically decreasing function of RG time $t$. On the contrary it has a flow similar to $M^{2} / \mu^{2}$, having a single minimum. But there is a region of RG time $t$ over which $M^{2} / \omega \mu^{2}<1$. This is because of the choice of initial condition $M_{*}^{2} / \mu_{*}^{2}=\rho_{*}$ we made. This will imply that there is a range of $t$ where this the scalar mode will be realised and will go on-shell, and outside this region it will remain unrealised. This can play a role in early universe to drive inflation. On the other hand the parameter $\rho_{*}$ can be chosen appropriately large in order to make this the scalar mode ghost (never going on-shell), while still having unitarity.

The running of the $\mathrm{VeV}$ also induces a running in the generated masses for the scalar and fermion. To analyse whether they are physically realised or not, we studied the behaviour of $m_{s}^{2} / \mu^{2}$ and $m_{f}^{2} / \mu^{2}$, respectively. It is seen that if we choose $\rho_{*}$ appropriately, then it is possible to make them not physically realisable. They never go on-shell but do effect the theory gravitationally.

The RG flow equations for the dimensionless couplings are gauge independent at one loop, however, at higher loop gauge dependence is expected to enter. The effective potential is gauge dependent, which is because the hessian carries gauge dependence. This gauge dependence then enters the $\mathrm{VeV}$ and any quantity which is related to $\mathrm{VeV}$ (induced Newton's constant and induced masses). In the paper we studied the problem in Landau gauge which is a physical gauge allowing propagation of only transverse modes and suppressing longitudinal ones. However, such gauge dependence is expected not to change qualitative features. This was explic- 
itly seen in the case of pure higher-derivative gravity without matter [5,6].

In the action appearing in the integrand for the Lorentzian path integral, the sign of coefficient of $C_{\mu \nu \rho \sigma}^{2}$ is taken to be negative while the sign of coefficient of $R^{2}$ is taken positive. This is done to avoid tachyons and make the poles (in broken phase) lie on real axis, the inspiration of which comes from past study done in [5-8]. Such a choice further offers necessary convergence in the Lorentzian path integral in the Feynman $+i \epsilon$-prescription. This sign choice then implies that the coupling $f^{2}$ is no longer asymptotically free different from what is seen in path integral defining a positive-definite Euclidean theory $[9,10,16,30-33]$ (which is an entirely different theory), but instead has a Landau pole. This Landau singularity, however, appears way beyond the point where the RG flow of all couplings stops. Also the occurrence of Landau singularity is very possibly a one-loop effect, as at higher loops the running of $f^{2}$ gets correction thereby hinting at the occurrence of fixed point $[5,6]$. Moreover, in this theory the dimensionless perturbative parameters $f^{2}, f^{2} / \omega$, $\xi \sqrt{f^{2}}, \xi \sqrt{f^{2} / \omega}, \lambda$ and $y_{t}$ remain small throughout the RG flow, thereby justifying the usage of a perturbative approximation and we do not enter non-perturbative regimes in our analysis.

The analysis done in the paper is on a flat background. This is because any generic background has locally flat regions allowed by the (strong) equivalence principle. Also when one is probing ultra-short distances, one can do the analysis on flat space-time, as the perturbative UV divergences are independent of the background. Moreover, the chances of the tensor ghost becoming physically realisable is more in UV (and nowhere else), therefore its avoidance is important in the UV case, which can be investigated on a flat spacetime. However, extrapolating flat space-time analysis in deep infrared can lead to erroneous conclusions. In performing this study the cosmological constant was put to zero, and was argued that it can be maintained to be zero in a supersymmetric framework. However, supersymmetry is broken in the IR case and this will generate the cosmological constant back again. Moreover, current observations also favour the existence of the cosmological constant in order to drive the accelerated expansion of the universe. Therefore a proper treatment of IR physics in a field theoretic language is needed. A possible direction would be to formulate the theory on a de Sitter background [92-95] (see also [96] and the references therein). This will give a more accurate description of the theory in the IR case.

The existence of tachyonic instability is a further indication that the chosen background of flat space-time is unstable. While this is an IR effect and an unavoidable outcome of gravitational theories coupled with scalar on flat spacetime, it signals the breakdown of flat space-time as the back- ground. It is expected that performing the study on a curved background might address these issues. For this, one would require a more accurate description of the formulation of field theory on curved background, and obtain the results in low energy limit. Alternatively one may have to incorporate non-localities appropriately to deal with IR physics $[97,98]$. This is a future direction and will be considered later.

It is interesting to wonder whether the RG equations gets modified when the decoupling of massive spin-2 ghost mode occurs, in the same manner as in flat space-time nongravitational QFTs where decoupling theorem exists [99]. In flat space-time QFTs a systematic order-by-order computation leads to decoupling of heavier modes in process occurring at energies less than the mass of heavy particle. This theorem has been suitably extended to the case of matter theories on curved background [83-85], where the beta function gets a correction in mass-dependent scheme. For spin- 2 fields the situation is a bit more involved, as incorporating mass in a diffeomorphism invariant manner is a tricky task. A possible way to achieve is by including higher-derivative terms in the action, which immediately brings in ghosts. If the ghost mass, however, is always above the energy scale, then ghosts get avoided due to decoupling. But this occurs in the quantum theory where RG running of the ghost mass is always above the energy scale. This implies an effective decoupling in the sense that this spin-2 ghost mode never goes on-shell and off-shell; it does not contribute to the imaginary part of amplitudes $[5,6]$. But currently it is unclear how such a decoupling will modify the RG flow equations of various parameters. This is worthy of further investigations and will be considered in future work.

Acknowledgements I would like to thank Prof. Ramesh Anishetty for several useful discussions and enlightening suggestions. I am thankful to Prof. Tianjun Li for support, encouragement and fruitful discussions. I would like to thank Nirmalya Kajuri, Nick Houston and Tuhin Mukherjee for useful discussions. I would like to thank IMSc for hosting my visit and providing hospitality, where initial stages of the work was done. I thank the referee for raising the point regarding the modification of RG equations under the decoupling of ghost modes.

Open Access This article is distributed under the terms of the Creative Commons Attribution 4.0 International License (http://creativecomm ons.org/licenses/by/4.0/), which permits unrestricted use, distribution, and reproduction in any medium, provided you give appropriate credit to the original author(s) and the source, provide a link to the Creative Commons license, and indicate if changes were made. Funded by SCOAP ${ }^{3}$.

\section{Appendix A: Expansions}

For the computation of the running of all couplings including wave-function renormalisation the background of flat spacetime is sufficient. However, for simplicity to compute the running of $R \phi^{2}$ coupling we employ heat-kernel methods, 
for which we take the background to be a de Sitter spacetime.

For the flat background we have $g_{\mu \nu}=\eta_{\mu \nu}+h_{\mu \nu}$,

$g^{\mu \nu}=\eta^{\mu \nu}-h^{\mu \nu}+h^{\mu}{ }_{\alpha} h^{\alpha \nu}+\cdots$.

The expansion of $\sqrt{-g}$ is

$\sqrt{-g}=1+\frac{1}{2} h+\frac{1}{8} h^{2}-\frac{1}{4} h_{\mu \nu} h^{\mu \nu}+\cdots$.

The tetrads $\left(e^{a}{ }_{\mu}\right)$ are related with the metric by the following:

$\eta_{a b} e^{a}{ }_{\mu} e^{b}{ }_{\nu}=g_{\mu \nu}$.

The inverse tetrads $\left(e_{a}{ }^{\mu}\right)$ are similarly related with the inverse metric. Using these relations one can work out the expansion of the tetrads and inverse tetrads in terms of the metric fluctuation field $h_{\mu \nu}$,

$e_{\mu}^{a}=\bar{e}_{\rho}^{a}\left(\delta_{\mu}^{\rho}+\frac{1}{2} h_{\mu}^{\rho}-\frac{1}{8} h_{\alpha}^{\rho} h_{\mu}^{\alpha}+\cdots\right)$,

$e_{a}^{\mu}=\bar{e}_{a}{ }^{\rho}\left(\delta_{\rho}{ }^{\mu}-\frac{1}{2} h_{\rho}{ }^{\mu}+\frac{3}{8} h_{\rho}{ }^{\alpha} h_{\alpha}{ }^{\mu}+\cdots\right)$.

As the determinant of tetrad $e$ is just $\sqrt{-g}$, its expansion is the same as in Eq. (A2).

The expansion of the Christoffel connection and spin connection can be performed by first writing them in terms of metric and tetrads respectively, and then using the expansion of metric and tetrad mentioned above to obtain their expansion.

The Christoffel connection is given by

$\Gamma_{\alpha}^{\mu}{ }_{\beta}=\frac{1}{2} g^{\mu \rho}\left[\partial_{\alpha} g_{\rho \beta}+\partial_{\beta} g_{\rho \alpha}-\partial_{\rho} g_{\alpha \beta}\right]$,

Its expansion in terms of the $h_{\mu \nu}$ around the flat background is

$$
\begin{aligned}
\Gamma_{\alpha}{ }_{\beta}= & \frac{1}{2}\left(\partial_{\alpha} h_{\beta}^{\mu}+\partial_{\beta} h_{\alpha}^{\mu}-\partial^{\mu} h_{\alpha \beta}\right) \\
& -\frac{1}{2} h^{\mu \rho}\left(\partial_{\alpha} h_{\rho \beta}+\partial_{\beta} h_{\rho \alpha}-\partial_{\rho} h_{\alpha \beta}\right)+\cdots .
\end{aligned}
$$

The spin connection $\omega_{\mu c d}$ for torsion-free space-time has a simple expression in terms of Christoffel connection

$\omega_{\mu}^{a d}=e^{d v} e^{a}{ }_{\lambda} \Gamma_{\mu}{ }^{\lambda} \nu-e^{d \nu} \partial_{\mu} e^{a}{ }_{\nu}$.

The Christoffel connection $\Gamma_{\alpha}{ }^{\mu}{ }_{\beta}$ can be expressed in terms of tetrads and its inverse. This when plugged in Eq. (A7) gives the spin connection solely in terms of the tetrads, inverse tetrads and derivative of tetrad,

$$
\begin{aligned}
\omega_{\mu}^{a d}= & \frac{1}{2}\left[e^{a \rho}\left(\partial_{\mu} e_{\rho}^{d}-\partial_{\rho} e_{\mu}^{d}\right)-e^{d \rho}\left(\partial_{\mu} e_{\rho}^{a}-\partial_{\rho} e_{\mu}^{a}\right)\right. \\
& \left.+e^{b}{ }_{\mu} e^{a \rho} e^{d \nu}\left(\partial_{\nu} e_{b \rho}-\partial_{\rho} e_{b \nu}\right)\right] .
\end{aligned}
$$

By plugging the expansion of the tetrads and inverse tetrads one can obtain the expansion of the spin connection in terms of the metric fluctuation fields,

$$
\begin{aligned}
\omega_{\mu}{ }^{a d}= & \left(\bar{e}^{a \theta} \bar{e}^{d \tau}-\bar{e}^{a \tau} \bar{e}^{d \theta}\right)\left[-\partial_{\theta} h_{\tau \mu}-\frac{1}{4} h_{\theta}{ }^{\rho} \partial_{\mu} h_{\tau \rho}\right. \\
& \left.+\frac{1}{2} h_{\tau \rho} \partial_{\theta} h_{\mu}^{\rho}+\frac{1}{2} h_{\theta}{ }^{\rho} \partial_{\rho} h_{\tau \mu}+\cdots\right] .
\end{aligned}
$$

The Riemann curvature tensor is $R_{\mu \nu}{ }^{\rho}{ }_{\sigma}=\partial_{\mu} \Gamma_{\nu}{ }^{\rho}{ }_{\sigma}-$ $\partial_{\nu} \Gamma_{\mu}{ }^{\rho}{ }_{\sigma}+\Gamma_{\mu}{ }_{\lambda}{ }_{\lambda} \Gamma_{\nu_{\sigma}}^{\lambda}-\Gamma_{\nu}{ }^{\rho}{ }_{\lambda} \Gamma_{\mu}{ }_{\sigma}^{\lambda}$. We are interested only in the expansion of the Ricci tensor and Ricci scalar. We have

$$
\begin{aligned}
& R_{\mu \nu}=\frac{1}{2}\left(\partial_{\rho} \partial_{\mu} h_{\nu}^{\rho}+\partial_{\rho} \partial_{\nu} h_{\mu}^{\rho}-\square h_{\mu \nu}-\partial_{\mu} \partial_{\nu} h\right) \\
& +\partial_{\rho} \Gamma_{\mu}^{(2)} \rho_{\nu}-\partial_{\mu} \Gamma_{\rho}^{(2)} \rho_{\nu} \\
& +\frac{1}{4}\left[\partial^{\lambda} h\left(\partial_{\mu} h_{\lambda \nu}+\partial_{\nu} h_{\lambda \mu}-\partial_{\lambda} h_{\mu \nu}\right)-\partial_{\mu} h^{\rho \sigma} \partial_{\nu} h_{\rho \sigma}\right. \\
& \left.-2 \partial^{\lambda} h^{\rho}{ }_{\mu} \partial_{\rho} h_{\lambda v}+2 \partial^{\lambda} h^{\rho}{ }_{\mu} \partial_{\lambda} h_{\rho v}\right] \text {, } \\
& R=\partial_{\mu} \partial_{\nu} h^{\mu \nu}-\square h-\frac{1}{2} h^{\mu \nu}\left(2 \partial_{\rho} \partial_{\mu} h_{\nu}^{\rho}-\square h_{\mu \nu}-\partial_{\mu} \partial_{\nu} h\right) \\
& +\eta^{\mu \nu}\left(\partial_{\rho} \Gamma_{\mu}^{(2)} \rho_{\nu}-\partial_{\mu} \Gamma_{\rho}^{(2)} \rho_{\nu}\right)+\frac{1}{4}\left[\partial^{\lambda}\left(2 \partial^{\sigma} h_{\lambda \sigma}-\partial_{\lambda} h\right)\right. \\
& \left.+\partial^{\rho} h^{\nu \lambda} \partial_{\rho} h_{\nu \lambda}-2 \partial^{\lambda} h^{\rho v} \partial_{\rho} h_{\lambda \nu}\right]
\end{aligned}
$$

\section{Appendix B: Projectors}

The metric fluctuation field $h_{\mu \nu}$ around a general background can be decomposed into various components by doing a transverse-traceless decomposition. This is equivalent to doing decomposition of a vector into transverse and longitudinal components. For the metric fluctuation field $h_{\mu \nu}$ around a flat background, this decomposition can be written in momentum space as

$$
\begin{aligned}
h_{\mu \nu}= & h_{\mu \nu}^{\mathrm{T}}+\iota\left(q_{\mu} \xi_{\nu}+q_{\nu} \xi_{\mu}\right) \\
& +\left(\eta_{\mu \nu}-\frac{q_{\mu} q_{\nu}}{q^{2}}\right) s+\frac{q_{\mu} q_{\nu}}{q^{2}} w .
\end{aligned}
$$

where the various components satisfies the following constraints:

$h_{\mu}^{\mathrm{T} \mu}=0, \quad q^{\mu} h_{\mu \nu}^{\mathrm{T}}=0, \quad q^{\mu} \xi_{\mu}=0$.

Here $h_{\mu \nu}^{\mathrm{T}}$ is a transverse-traceless symmetric tensor, $\xi_{\mu}$ is a transverse vector and $s$ and $w$ are two scalars. This decomposition can be neatly written by making use of flat space-time projectors, which projects various components of $h_{\mu \nu}$ field into $h_{\mu \nu}^{\mathrm{T}}, \xi_{\mu}, s$ and $w$, respectively. These projectors are written in terms of the following two projectors:

$L_{\mu \nu}=\frac{q_{\mu} q_{\nu}}{q^{2}}, \quad T_{\mu \nu}=\eta_{\mu \nu}-\frac{q_{\mu} q_{\nu}}{q^{2}}$.

These are basically the projector for projecting out various components of a vector field. They satisfy $q^{\mu} T_{\mu \nu}=0$ and 
$q^{\mu} L_{\mu \nu}=q_{\nu}$. Using them the projectors for the rank-2 tensor field can be constructed. These are given by

$\left(P_{2}\right)_{\mu \nu}{ }^{\alpha \beta}=\frac{1}{2}\left[{T_{\mu}}^{\alpha} T_{\nu}{ }^{\beta}+T_{\mu}{ }^{\beta}{T_{\nu}}^{\alpha}\right]-\frac{1}{d-1} T_{\mu \nu} T^{\alpha \beta}$
$\left(P_{1}\right)_{\mu \nu}{ }^{\alpha \beta}=\frac{1}{8}\left[{T_{\mu}}^{\alpha}{L_{\nu}}^{\beta}+T_{\mu}{ }^{\beta}{L_{\nu}}^{\alpha}+T_{\nu}{ }^{\alpha} L_{\mu}{ }^{\beta}+T_{\nu}{ }^{\beta} L_{\mu}{ }^{\alpha}\right]$

$\left(P_{S}\right)_{\mu \nu}{ }^{\alpha \beta}=\frac{1}{d-1} T_{\mu \nu} T^{\alpha \beta}$,

$\left(P_{w}\right)_{\mu \nu}^{\alpha \beta}=L_{\mu \nu} L^{\alpha \beta}$.

The projectors for spin-2, spin-1, spin-s and spin-w form an orthogonal set. In the scalar sector there are two more projectors (which are not projectors in the strict sense), which along with spin-s and spin-w projectors form a complete set. They are given by

$\left(P_{s w}\right)_{\mu \nu}^{\alpha \beta}=\frac{1}{\sqrt{d-1}} T_{\mu \nu} L^{\alpha \beta}$
$\left(P_{w s}\right)_{\mu \nu}^{\alpha \beta}=\frac{1}{\sqrt{d-1}} L_{\mu \nu} T^{\alpha \beta}$.

The projectors in Eqs. (B4)-(B7) forms a complete set in the sense that their sum is unity. We have

$\left(P_{2}\right)_{\mu \nu}{ }^{\rho \sigma}+\left(P_{1}\right)_{\mu \nu}{ }^{\rho \sigma}+\left(P_{S}\right)_{\mu \nu}{ }^{\rho \sigma}+\left(P_{w}\right)_{\mu \nu}{ }^{\rho \sigma}=\delta_{\mu \nu}^{\rho \sigma}$,

where $\delta_{\mu \nu}^{\rho \sigma}=1 / 2\left(\delta_{\mu}^{\rho} \delta_{\nu}^{\sigma}+\delta_{\nu}^{\rho} \delta_{\mu}^{\sigma}\right)$. Each of these projectors when act on $h_{\mu \nu}$ projects out various spin components of the tensor field. We have

$$
\begin{aligned}
& \left(P_{2}\right)_{\mu \nu}{ }^{\rho \sigma} h_{\rho \sigma}=h_{\mu \nu}^{\mathrm{T}}, \quad\left(P_{1}\right)_{\mu \nu}{ }^{\rho \sigma} h_{\rho \sigma}=\iota\left(q_{\mu} \xi_{\nu}+q_{\nu} \xi_{\mu}\right), \\
& \left(P_{s}\right)_{\mu \nu}{ }^{\rho \sigma} h_{\rho \sigma}=(d-1) T_{\mu \nu} s, \quad\left(P_{w}\right)_{\mu \nu}{ }^{\rho \sigma} h_{\rho \sigma}=L_{\mu \nu} w .
\end{aligned}
$$

If the projectors $P_{2}, P_{1}, P_{s}$ and $P_{w}$ are written as $P_{22}, P_{11}$, $P_{s s}$ and $P_{w w}$ respectively, then all the projectors (including $P_{s w}$ and $\left.P_{w s}\right)$ satisfy the following algebra:

$P_{i j} P_{m n}=\delta_{j m} P_{i n}$,

where $i, j, m$ and $n=\{2,1, s, w\}$.

\section{Appendix C: Matter propagator and vertices}

Here we write the propagator for matter fields and vertices of the action given in Eq. (2). These are obtained by doing the second variation of the action with respect to various fields. The first line of Eq. (2) gives the graviton propagator which is mentioned in Eq. (17), while the second line of Eq. (2) gives the propagator for the matter fields and various gravitonmatter, matter-matter vertices. In the following we will be obtaining them one by one.

\section{C.1. Propagators for Matter fields}

Here we write the inverse propagators for the various matter fields. These are obtained by doing the second variation of the action of the theory with respect to various fields. The mixed terms in such kind of variation will be treated as interaction terms. From the second variation of the action given in Eq. (19) one can pick the terms corresponding to the scalar and fermion propagator. The operator whose inverse corresponds to the scalar propagator is

$\Delta_{s}=-\partial^{2}$

In the case of fermions the relevant inverse operator is given by

$\left(\Delta_{F}\right)_{a b}=i \gamma_{a b}^{\rho} \partial_{\rho}$.

\section{C.2. Vertices}

Here we specify the various vertices that are relevant for our one-loop computations. These can be categorised in three parts: (a) vertex with two internal graviton lines, (b) vertex with one internal graviton line and one internal matter line, and (c) vertex with two internal matter lines.

a. Gravity-gravity

In these vertices there are two internal graviton lines. In the following the term $V^{\mu \nu \rho \sigma}$ comes from the scalar-field action, while the term $U^{\mu \nu \rho \sigma}$ comes from fermion field action. The vertices are depicted in Fig. 15. We have

$$
\begin{aligned}
& \int \mathrm{d}^{d} x h_{\mu \nu}\left(V^{\mu \nu \rho \sigma}+U^{\mu \nu \rho \sigma}\right) h_{\rho \sigma} \\
& V^{\mu \nu \rho \sigma}=\frac{1}{4}\left(\eta^{\mu \nu} \eta^{\rho \sigma}-\eta^{\mu \rho} \eta^{\nu \sigma}-\eta^{\mu \sigma} \eta^{\nu \rho}\right) \\
& \times\left\{\frac{1}{2}(\partial \varphi)^{2}-\frac{\lambda}{4} \varphi^{4}\right\}-\frac{1}{2} \eta^{\rho \sigma} \partial^{\mu} \varphi \partial^{\nu} \varphi+\eta^{\sigma v} \partial^{\mu} \varphi \partial^{\rho} \varphi-\frac{1}{2} \xi \varphi^{2} \\
& \times\left\{-\eta^{\nu \sigma} \partial^{\mu} \partial^{\rho}+\frac{1}{4}\left(\eta^{\mu \rho} \eta^{\nu \sigma}+\eta^{\mu \sigma} \eta^{\nu \rho}-2 \eta^{\mu \nu} \eta^{\rho \sigma}\right) \square\right. \\
& \left.+\eta^{\mu \nu} \partial^{\rho} \partial^{\sigma}\right\}-\xi\left\{\varphi \partial^{\mu} \varphi\left(\eta^{\nu \rho} \partial^{\sigma}-2 \eta^{\rho \sigma} \partial^{v}\right)\right. \\
& \left.-\frac{1}{4} \varphi \partial^{\alpha} \varphi\left(3\left(\eta^{\mu \rho} \eta^{\nu \sigma}+\eta^{\mu \sigma} \eta^{\nu \rho}\right)-2 \eta^{\mu \nu} \eta^{\rho \sigma}\right) \partial_{\alpha}\right\} \\
& +\frac{1}{2} \xi\left(\partial^{v} \varphi \partial^{\beta} \varphi+\varphi \partial^{v} \partial^{\beta} \varphi\right)\left(\eta^{\mu \rho} \delta_{\beta}^{\sigma}+\eta^{\mu \sigma} \delta_{\beta}^{\rho}\right), \\
& U^{\mu \nu \rho \sigma}=\frac{i}{4}\left(\eta^{\mu \nu} \eta^{\rho \sigma}-\eta^{\mu \rho} \eta^{\nu \sigma}-\eta^{\mu \sigma} \eta^{\nu \rho}\right) \bar{\theta} \gamma^{\tau} \partial_{\tau} \theta \\
& -\frac{i}{2} \eta^{\mu \nu} \bar{\theta} \gamma^{\rho} \partial^{\sigma} \theta+\frac{3 i}{4} \eta^{\sigma \nu} \bar{\theta} \gamma^{\rho} \partial^{\mu} \theta \\
& -\frac{i}{8} \eta^{\sigma v} \bar{\theta} \gamma^{\tau}\left[\gamma^{\mu}, \gamma^{\rho}\right] \theta \partial_{\tau} \\
& +\frac{i}{4} \bar{\theta} \gamma^{\rho}\left[\gamma^{\mu}, \gamma^{\sigma}\right] \theta \partial^{\nu}-\frac{i}{4} \eta^{\nu \sigma} \partial_{\alpha}\left\{\bar{\theta} \gamma^{\rho}\left[\gamma^{\alpha}, \gamma^{\mu}\right] \theta\right\}
\end{aligned}
$$



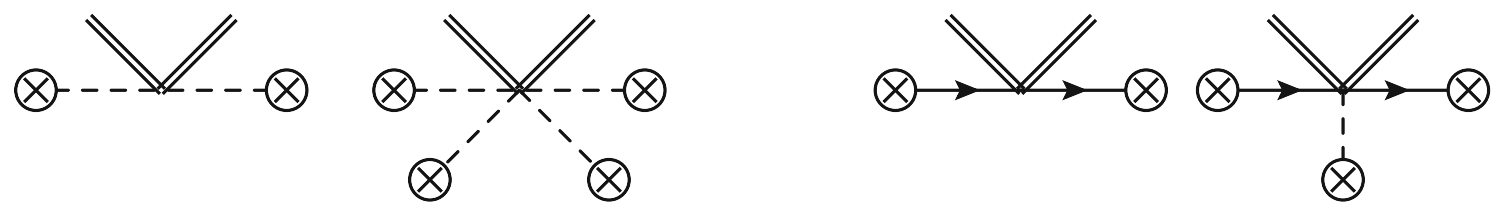

Fig. 15 Various vertices containing two internal graviton lines. Here the dashed line is the scalar, the solid line with arrow is the fermion, while the double line depicts the graviton. The lines ending with a circle containing a cross are external legs

$$
\begin{aligned}
& -\frac{i}{4} \eta^{\mu \nu} \bar{\theta} \gamma^{\rho}\left[\gamma^{\alpha}, \gamma^{\sigma}\right] \theta \partial_{\alpha} \\
& -\frac{1}{4} y_{t} \varphi\left(\eta^{\mu \nu} \eta^{\rho \sigma}-\eta^{\mu \rho} \eta^{\nu \sigma}-\eta^{\mu \sigma} \eta^{\nu \rho}\right) \bar{\theta} \theta .
\end{aligned}
$$

\section{b. Gravity-scalar}

Here we write the vertex which has one internal graviton line and one internal scalar line. This vertex gets contributions from both scalar and fermion field actions. These vertices are depicted in Fig. 16. We have

$$
\begin{aligned}
& \int \mathrm{d}^{d} x\left[h_{\rho \sigma}\left(V_{h \phi}\right)^{\rho \sigma} \chi+\chi\left(V_{\phi h}\right)^{\mu \nu} h_{\mu \nu}\right] \\
& \left(V_{h \phi}\right)_{\rho \sigma}=-\frac{\lambda}{2} \varphi^{2} \eta_{\rho \sigma} \varphi+\frac{1}{2} \eta_{\rho \sigma} \partial_{\alpha} \varphi \partial^{\alpha} \\
& \quad-\partial_{\rho} \varphi \partial_{\sigma}-\xi\left\{\partial_{\rho} \partial_{\sigma} \varphi+\partial_{\rho} \varphi \partial_{\sigma}+\partial_{\sigma} \varphi \partial_{\rho}+\varphi \partial_{\rho} \partial_{\sigma}\right\} \\
& \quad+\xi \eta_{\rho \sigma}\left(\square \varphi+2 \partial_{\alpha} \varphi \partial^{\alpha}+\varphi \square\right)-\frac{1}{2} \eta_{\rho \sigma} y_{t} \bar{\theta} \theta, \\
& \left(V_{\phi h}\right)_{\mu \nu}=-\frac{\lambda}{2} \lambda \varphi^{2} \eta_{\mu \nu} \varphi-\frac{1}{2} \eta_{\mu \nu} \partial_{\beta} \varphi \partial^{\beta} \\
& \quad-\frac{1}{2} \eta_{\mu \nu} \square \varphi+\partial_{\mu} \varphi \partial_{\nu}+\partial_{\mu} \partial_{\nu} \varphi \\
& -\xi \varphi\left(\partial_{\mu} \partial_{\nu}-\eta_{\mu \nu} \square\right)-\frac{1}{2} \eta_{\mu \nu} y_{t} \bar{\theta} \theta .
\end{aligned}
$$

\section{c. Gravity-fermion}

Here we write the vertex that contain one internal graviton line and one internal fermion line. This vertex comes only from the fermion field action. The vertices are depicted in Fig. 17. We have

$$
\begin{aligned}
& \int \mathrm{d}^{d} x\left[\bar{\eta}_{d}\left(V_{\bar{\psi} h}\right)_{d}^{\rho \sigma} h_{\rho \sigma}+h_{\mu \nu}\left(V_{h \bar{\psi}}^{\mathrm{T}}\right)_{c}^{\mu \nu} \bar{\eta}_{c}^{\mathrm{T}}\right], \\
& \left(V_{\bar{\psi} h}\right)_{d \rho \sigma}=\frac{i}{2}\left(\eta_{\rho \sigma} \gamma_{d e}^{\tau} \partial_{\tau} \theta_{e}-\left(\gamma_{\rho}\right)_{d e} \partial_{\nu} \theta_{e}\right) \\
& \quad-\frac{i}{4}\left(\gamma_{\rho}\left[\gamma^{\alpha}, \gamma_{\sigma}\right]\right)_{d e} \theta_{e} \partial_{\alpha}-\frac{y_{t}}{2} \varphi \eta_{\rho \sigma} \theta_{d}, \\
& \left(V_{h \bar{\psi}}^{\mathrm{T}}\right)_{c}^{\mu \nu}=-\frac{i}{2}\left\{\eta^{\mu \nu} \partial_{\tau} \theta_{e}\left(\gamma^{T \tau}\right)_{e c}-\left(\partial^{\nu} \theta_{e}^{\mathrm{T}}\right) \gamma_{e c}^{T \mu}\right\} \\
& -\frac{i}{4}\left(\partial_{\alpha} \theta_{e}^{\mathrm{T}}\right)\left\{\left[\gamma^{\mu T}, \gamma^{\alpha T}\right] \gamma^{\nu T}\right\}_{e c} \\
& -\frac{i}{4}\left(\theta_{e}^{\mathrm{T}}\right)\left\{\left[\gamma^{\mu T}, \gamma^{\alpha T}\right] \gamma^{\nu T}\right\}_{e c} \partial_{\alpha}+\frac{1}{4} \eta^{\mu \nu} y_{t} \varphi \theta_{c}^{\mathrm{T}},
\end{aligned}
$$<smiles>CC(C)(O)Br</smiles>

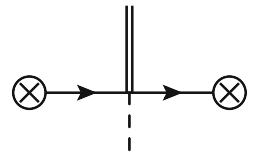

Fig. 16 Various vertices containing one internal graviton line and one internal scalar leg. Here the dashed line is the scalar, the solid line with arrow is the fermion, while the double line depicts the graviton. The lines ending with a circle containing a cross are external legs

$$
\begin{aligned}
& \int \mathrm{d}^{d} x\left[h_{\mu \nu}\left(V_{h \psi}\right)_{a}^{\mu \nu} \eta_{a}+\eta_{a}^{\mathrm{T}}\left(V_{\psi h}^{\mathrm{T}}\right)^{\rho \sigma} h_{\rho \sigma}\right] \\
& \left(V_{h \psi}\right)_{c}^{\mu \nu}=\frac{i}{2}\left\{\eta^{\mu \nu} \bar{\theta}_{e} \gamma_{e c}^{\tau} \partial_{\tau}-\bar{\theta}_{e} \gamma_{e c}^{\nu} \partial^{\mu}\right\} \\
& \quad+\frac{i}{4}\left\{\left(\partial_{\alpha} \bar{\theta}_{e}\right)\left(\gamma^{\nu}\left[\gamma^{\alpha}, \gamma^{\mu}\right]\right)_{e c}+\bar{\theta}_{e}\left(\gamma^{\nu}\left[\gamma^{\alpha}, \gamma^{\mu}\right]\right)_{e c} \partial_{\alpha}\right\} \\
& \quad-\frac{y_{t}}{4} \eta^{\mu \nu} \varphi \bar{\theta}_{c}, \\
& \left(V_{\psi h}^{\mathrm{T}}\right)_{b}^{\rho \sigma}=\frac{i}{2}\left\{\eta^{\rho \sigma} \gamma_{b e}^{T \tau}\left(\bar{\theta}_{e}^{\mathrm{T}} \partial_{\tau}+\partial_{\tau} \bar{\theta}_{e}^{\mathrm{T}}\right)\right. \\
& \left.\quad-\gamma_{b e}^{T \sigma}\left(\bar{\theta}_{e}^{\mathrm{T}} \partial^{\rho}+\partial^{\rho} \bar{\theta}_{e}^{\mathrm{T}}\right)\right\}+\frac{i}{4}\left(\left[\gamma^{T \rho}, \gamma^{T \alpha}\right] \gamma^{T \sigma}\right)_{b e} \bar{\theta}_{e}^{\mathrm{T}} \partial_{\alpha} \\
& +\frac{y_{t}}{2} \eta^{\rho \sigma} \bar{\theta}_{b}^{\mathrm{T}} \varphi .
\end{aligned}
$$

\section{d Matter-matter}

Here we write the vertices which has two internal matter lines. These will be either both scalar lines, one scalar and one fermion line or both fermion lines. These vertices are depicted in Fig. 18. We have

$$
\begin{array}{ll}
\int \mathrm{d}^{d} x\left[-\chi\left(V_{s}\right) \chi+\bar{\eta}_{a}\left(V_{\bar{\psi} \psi}\right)^{a b} \eta_{b}+\eta_{a}^{\mathrm{T}}\left(V_{\bar{\psi} \psi}^{\mathrm{T}}\right)^{a b} \bar{\eta}_{b}^{\mathrm{T}}\right. & \\
\left.\quad+\bar{\eta}_{a}\left(V_{\bar{\psi} \phi}\right)^{a} \chi+\chi\left(V_{\bar{\psi} \phi}^{\mathrm{T}}\right)^{b} \bar{\eta}_{b}^{\mathrm{T}}+\chi\left(V_{\phi \psi}\right)^{b} \eta_{b}+\eta_{a}^{\mathrm{T}}\left(V_{\phi \psi}^{\mathrm{T}}\right)^{a} \chi\right], \\
V_{s}=3 \lambda \varphi^{2}, & (\mathrm{C} 11) \\
\left(V_{\bar{\psi} \psi}\right)^{a b}=-y_{t} \varphi \delta^{a b}, \quad\left(V_{\bar{\psi} \psi}^{\mathrm{T}}\right)^{a b}=y_{t} \varphi \delta^{a b}, & (\mathrm{C} 12) \\
\left(V_{\bar{\psi} \phi}\right)^{a}=-y_{t} \theta^{a}, \quad\left(V_{\bar{\psi} \phi}^{\mathrm{T}}\right)^{a}=y_{t} \theta^{T a}, & (\mathrm{C} 13) \\
\left(V_{\phi \psi}\right)^{b}=-y_{t} \bar{\theta}^{b}, \quad\left(V_{\phi \psi}^{\mathrm{T}}\right)^{b}=y_{t} \bar{\theta}^{T b} . &
\end{array}
$$

\section{Appendix D: Cubic equation}

Here we will consider the roots of generic cubic equation with real coefficients. Such an equation emerges in Sect. 3 while 

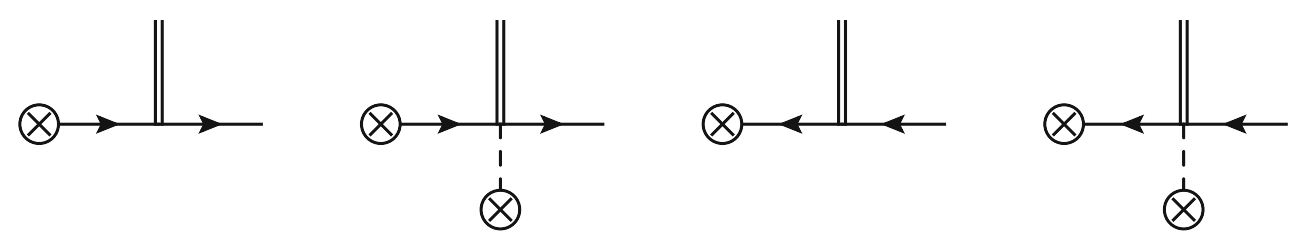

Fig. 17 Various vertices containing one internal graviton line and one internal fermion leg. Here the dashed line is the scalar, solid line with arrow is fermion, while the double line depicts graviton. The lines ending with a circle containing a cross are external legs
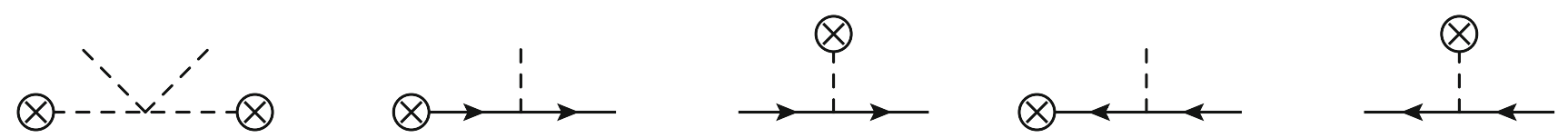

Fig. 18 Various vertices containing two internal matter legs (scalar-scalar, scalar-fermion, fermion-fermion). Here the dashed line is the scalar while the solid line with arrow is the fermion. The lines ending with a circle containing a cross are external legs

computing the contribution to the scalar effective potential from the scalar sector of the theory. In order to compute the contribution of the scalar to the effective potential we need analyse a cubic equation in the $-\square$ operator written in Eq. (62). In this section we will consider a generic cubic equation of the form

$a z^{3}+b z^{2}+c z+d=0$.

By the change of variable $z=u-b / 3 a$, this equation becomes a depressed cubic,

$u^{3}-\frac{\Delta_{0}}{3 a^{2}} u+\frac{\Delta_{1}}{27 a^{3}}=0$,

$\Delta_{0}=b^{2}-3 a c, \quad \Delta_{1}=2 b^{3}-9 a b c+27 d a^{2}$.

By choosing $u=v+\Delta_{0} / 9 a^{2} v$, this equation can be converted into a quadratic equation in $v^{3}$,

$v^{6}+\frac{\Delta_{1}}{27 a^{3}} v^{3}+\frac{\Delta_{0}^{3}}{729 a^{6}}=0$.

This quadratic equation can be solved by known algebraic methods and has two roots. The nature of the roots can be determined by the sign of the discriminant of this quadratic equation,

$-27 a^{2} \Delta=\Delta_{1}^{2}-4 \Delta_{0}^{3}$,

where $\Delta$ was also defined in Eq. (63). The two roots of the quadratic equation will be given by

$v^{3}=\frac{-\Delta_{1} \pm \sqrt{\Delta_{1}^{2}-4 \Delta_{0}^{3}}}{54 a^{3}}=\frac{1}{27 a^{3}} l_{1,2}^{3}$.

This can be solved easily by taking the cube root. Here there will be three roots for $v$. Corresponding to each cube root we have a root for the Eq. (D1), which is obtained by plugging $v$ back into $u$ and $z$. The three roots will be given in terms of $l_{1}$ and $l_{2}$,

$$
\begin{aligned}
& z_{1}=\frac{1}{3 a}\left(-b+l_{1}+l_{2}\right), \\
& z_{2}=\frac{1}{3 a}\left(-b+e^{2 \pi i / 3} l_{1}+e^{4 \pi i / 3} l_{2}\right), \\
& z_{3}=\frac{1}{3 a}\left(-b+e^{4 \pi i / 3} l_{1}+e^{2 \pi i / 3} l_{2}\right) .
\end{aligned}
$$

In the case when the discriminant $\Delta_{1}^{2}-4 \Delta_{0}^{3}>0$, we have one real root and a complex conjugate pair. In this case we can write the exponentials in terms of sine and cosine functions. One can then write the real and imaginary part of these roots. This complex conjugate pair can be written in polar form also with

$$
\begin{aligned}
& r=\frac{1}{3 a} \sqrt{b^{2}+l_{1}^{2}+l_{2}^{2}-2 \cos \left(\frac{2 \pi}{3}\right)\left(b l_{1}+b l_{2}+l_{1} l_{2}\right)}, \\
& \tan \theta=\frac{\sin (2 \pi / 3)\left(l_{1}-l_{2}\right)}{-b+\cos (2 \pi / 3)\left(l_{1}+l_{2}\right)}, \\
& \text { with }-\frac{\pi}{2} \leq \theta \leq \frac{\pi}{2} .
\end{aligned}
$$

In the case when the discriminant $\Delta_{1}^{2}-4 \Delta_{0}^{3}<0$, we have a complex conjugate pair of the roots for $v^{3}$, meaning $l_{1}$ and $l_{2}$ will be complex conjugate. This will imply that the expressions for $z_{1}, z_{2}$ and $z_{3}$ are real. However, now $\theta$ appearing in Eq. (D8) will be imaginary.

\section{References}

1. K.S. Stelle, Classical gravity with higher derivatives. Gen. Relativ. Gravit. 9, 353 (1978). doi:10.1007/BF00760427

2. K.S. Stelle, Renormalization of higher derivative quantum gravity. Phys. Rev. D 16, 953 (1977). doi:10.1103/PhysRevD.16.953

3. A. Salam, J.A. Strathdee, Remarks on high-energy stability and renormalizability of gravity theory. Phys. Rev. D 18, 4480 (1978). doi:10.1103/PhysRevD.18.4480

4. J. Julve, M. Tonin, Quantum gravity with higher derivative terms. Nuovo Cim. B 46, 137 (1978). doi:10.1007/BF02748637 
5. G. Narain, R. Anishetty, Short distance freedom of quantum gravity. Phys. Lett. B 711, 128 (2012). doi:10.1016/j.physletb.2012.03. 070. arXiv:1109.3981 [hep-th]

6. G. Narain, R. Anishetty, Unitary and renormalizable theory of higher derivative gravity. J. Phys. Conf. Ser. 405, 012024 (2012). doi:10.1088/1742-6596/405/1/012024. arXiv:1210.0513 [hep-th]

7. G. Narain, R. Anishetty, Charge renormalization due to graviton loops. JHEP 1307, 106 (2013). doi:10.1007/JHEP07(2013)106. arXiv:1211.5040 [hep-th]

8. G. Narain, R. Anishetty, Running couplings in quantum theory of gravity coupled with gauge fields. JHEP 1310, 203 (2013). doi:10. 1007/JHEP10(2013)203. arXiv:1309.0473 [hep-th]

9. E.S. Fradkin, A.A. Tseytlin, Renormalizable asymptotically free quantum theory of gravity. Phys. Lett. 104B, 377 (1981). doi:10. 1016/0370-2693(81)90702-4

10. E.S. Fradkin, A.A. Tseytlin, Renormalizable asymptotically free quantum theory of gravity. Nucl. Phys. B 201, 469 (1982). doi:10. 1016/0550-3213(82)90444-8

11. L. Alvarez-Gaume, A. Kehagias, C. Kounnas, D. Lust, A. Riotto, Aspects of quadratic gravity. Fortschr. Phys. 64(2-3), 176 (2016). doi:10.1002/prop.201500100. arXiv:1505.07657 [hep-th]

12. D.M. Capper, M.J. Duff, Trace anomalies in dimensional regularization. Nuovo Cim. A 23, 173 (1974). doi:10.1007/BF02748300

13. S. Deser, M.J. Duff, C.J. Isham, Nonlocal conformal anomalies. Nucl. Phys. B 111, 45 (1976). doi:10.1016/0550-3213(76)90480-6

14. M.J. Duff, Observations on conformal anomalies. Nucl. Phys. B 125, 334 (1977). doi:10.1016/0550-3213(77)90410-2

15. N.H. Barth, S.M. Christensen, Quantizing fourth order gravity theories. 1. The functional integral. Phys. Rev. D 28, 1876 (1983). doi:10.1103/PhysRevD.28.1876

16. I.G. Avramidi, A.O. Barvinsky, Asymptotic freedom in higher derivative quantum gravity. Phys. Lett. 159B, 269 (1985). doi:10. 1016/0370-2693(85)90248-5

17. I.L. Buchbinder, O.K. Kalashnikov, I.L. Shapiro, V.B. Vologodsky, J.J. Wolfengaut, The stability of asymptotic freedom in grand unified models coupled to $R^{2}$ gravity. Phys. Lett. B 216, 127 (1989). doi:10.1016/0370-2693(89)91381-6

18. I.L. Shapiro, Asymptotic behavior of effective Yukawa coupling constants in quantum $R^{2}$ gravity with matter. Class. Quantum Gravity 6, 1197 (1989). doi:10.1088/0264-9381/6/8/019

19. S.D. Odintsov, The parametrization invariant and gauge invariant effective actions in quantum field theory. Fortschr. Phys. 38, 371 (1990)

20. E. Elizalde, S.D. Odintsov, A. Romeo, Improved effective potential in curved space-time and quantum matter, higher derivative gravity theory. Phys. Rev. D 51, 1680 (1995). doi:10.1103/PhysRevD.51. 1680. arXiv:hep-th/9410113

21. E. Elizalde, C.O. Lousto, S.D. Odintsov, A. Romeo, GUTs in curved space-time: running gravitational constants, Newtonian potential and the quantum corrected gravitational equations. Phys. Rev. D 52, 2202 (1995). doi:10.1103/PhysRevD.52.2202. arXiv:hep-th/9504014

22. E. Elizalde, S.D. Odintsov, A. Romeo, Manifestations of quantum gravity in scalar QED phenomena. Phys. Rev. D 51, 4250 (1995). doi:10.1103/PhysRevD.51.4250. arXiv:hep-th/9410028

23. I.L. Buchbinder, S.D. Odintsov, I.L. Shapiro, Effective Action in Quantum Gravity (Bristol, IOP, 1992), p. 413

24. G. de Berredo-Peixoto, I.L. Shapiro, Conformal quantum gravity with the Gauss-Bonnet term. Phys. Rev. D 70, 044024 (2004). doi:10.1103/PhysRevD.70.044024. arXiv:hep-th/0307030

25. G. de Berredo-Peixoto, I.L. Shapiro, Higher derivative quantum gravity with Gauss-Bonnet term. Phys. Rev. D 71, 064005 (2005). doi:10.1103/PhysRevD.71.064005. arXiv:hep-th/0412249

26. A. Salvio, A. Strumia, Agravity. JHEP 1406, 080 (2014). doi:10. 1007/JHEP06(2014)080. arXiv:1403.4226 [hep-ph]
27. A. Salvio, Solving the standard model problems in softened gravity. Phys. Rev. D 94(9), 096007 (2016). doi:10.1103/PhysRevD.94. 096007. arXiv:1608.01194 [hep-ph]

28. K. Kannike, G. Htsi, L. Pizza, A. Racioppi, M. Raidal, A. Salvio, A. Strumia, Dynamically induced Planck scale and inflation. JHEP 1505, 065 (2015). doi:10.1007/JHEP05(2015)065. arXiv:1502.01334 [astro-ph.CO]

29. A. Salvio, A. Strumia, Quantum mechanics of 4-derivative theories. Eur. Phys. J. C 76(4), 227 (2016). doi:10.1140/epjc/ s10052-016-4079-8. arXiv:1512.01237 [hep-th]

30. M.B. Einhorn, D.R.T. Jones, Naturalness and dimensional transmutation in classically scale-invariant gravity. JHEP 1503, 047 (2015). doi:10.1007/JHEP03(2015)047. arXiv:1410.8513 [hep-th]

31. T. Jones, M. Einhorn, Quantum gravity and dimensional transmutation. PoS Planck 2015, 061 (2015)

32. M.B. Einhorn, D.R.T. Jones, Induced gravity I: real scalar field. JHEP 1601, 019 (2016). doi:10.1007/JHEP01(2016)019. arXiv: 1511.01481 [hep-th]

33. M.B. Einhorn, D.R.T. Jones, Induced gravity II: grand unification. JHEP 1605, 185 (2016). doi:10.1007/JHEP05(2016)185. arXiv: 1602.06290 [hep-th]

34. B. Holdom, J. Ren, QCD analogy for quantum gravity. Phys. Rev. D 93(12), 124030 (2016). doi:10.1103/PhysRevD.93.124030. arXiv: 1512.05305 [hep-th]

35. B. Holdom, J. Ren, Quadratic gravity: from weak to strong. Int. J. Mod. Phys. D 25(12), 1643004 (2016). doi:10.1142/ S0218271816430045. arXiv:1605.05006 [hep-th]

36. Y.B. Zeldovich, Cosmological constant and elementary particles. JETP Lett. 6, 316 (1967) [Pisma Zh. Eksp. Teor. Fiz. 6, 883 (1967)]

37. A.D. Sakharov, Vacuum quantum fluctuations in curved space and the theory of gravitation. Sov. Phys. Dokl. 12, 1040 (1968) [Dokl. Akad. Nauk Ser. Fiz. 177, 70 (1967)] [Sov. Phys. Usp. 34, 394 (1991)] [Gen. Relativ. Gravit. 32, 365 (2000)]

38. Y. Fujii, Scalar-tensor theory of gravitation and spontaneous breakdown of scale invariance. Phys. Rev. D 9, 874 (1974). doi:10.1103/ PhysRevD.9.874

39. E.M. Chudnovsky, The spontaneous conformal symmetry breaking and Higgs model. Theor. Math. Phys. 35, 538 (1978). doi:10.1007/ BF01036453 [Teor. Mat. Fiz. 35, 398 (1978)]

40. A. Zee, A broken symmetric theory of gravity. Phys. Rev. Lett. 42, 417 (1979). doi:10.1103/PhysRevLett.42.417

41. S.L. Adler, A formula for the induced gravitational constant. Phys. Lett. B 95, 241 (1980). doi:10.1016/0370-2693(80)90478-5

42. S.L. Adler, Order R vacuum action functional in scalar free unified theories with spontaneous scale breaking. Phys. Rev. Lett. 44, 1567 (1980). doi:10.1103/PhysRevLett.44.1567

43. S.L. Adler, Induced gravitation. AIP Conf. Proc. 68, 915 (1980). doi:10.1063/1.2948651

44. A. Zee, Spontaneously generated gravity. Phys. Rev. D 23, 858 (1981). doi:10.1103/PhysRevD.23.858

45. S.L. Adler, Einstein gravity as a symmetry breaking effect in quantum field theory. Rev. Mod. Phys. 54, 729 (1982). doi:10.1103/ RevModPhys.54.729 [Erratum: Rev. Mod. Phys. 55, 837 (1983)]

46. S.R. Coleman, E.J. Weinberg, Radiative corrections as the origin of spontaneous symmetry breaking. Phys. Rev. D 7, 1888 (1973). doi:10.1103/PhysRevD.7.1888

47. R.I. Nepomechie, Einstein gravity as the low-energy effective theory of Weyl gravity. Phys. Lett. 136B, 33 (1984). doi:10.1016/ 0370-2693(84)92050-1

48. A. Zee, Einstein gravity emerging from quantum Weyl gravity. Ann. Phys. 151, 431 (1983). doi:10.1016/0003-4916(83)90286-5

49. I.L. Buchbinder, Mechanism for induction of Einstein gravitation. Sov. Phys. J. 29, 220 (1986). doi:10.1007/BF00891883

50. I.L. Shapiro, G. Cognola, Interaction of low-energy induced gravity with quantized matter and phase transition induced to curvature. 
Phys. Rev. D 51, 2775 (1995). doi:10.1103/PhysRevD.51.2775. arXiv:hep-th/9406027

51. R. Floreanini, R. Percacci, Average effective potential for the conformal factor. Nucl. Phys. B 436, 141 (1995). doi:10.1016/ 0550-3213(95)00479-C. arXiv:hep-th/9305172

52. R. Floreanini, R. Percacci, The renormalization group flow of the dilaton potential. Phys. Rev. D 52, 896 (1995). doi:10.1103/ PhysRevD.52.896. arXiv:hep-th/9412181

53. F. Cooper, G. Venturi, Cosmology and broken scale invariance. Phys. Rev. D 24, 3338 (1981). doi:10.1103/PhysRevD.24.3338

54. F. Finelli, A. Tronconi, G. Venturi, Dark energy, induced gravity and broken scale invariance. Phys. Lett. B 659, 466 (2008). doi:10. 1016/j.physletb.2007.11.053. arXiv:0710.2741 [astro-ph]

55. C. Ford, D.R.T. Jones, P.W. Stephenson, M.B. Einhorn, The effective potential and the renormalization group. Nucl. Phys. B 395, 17 (1993). doi:10.1016/0550-3213(93)90206-5. arXiv:hep-lat/9210033

56. R. Percacci, Asymptotic safety, in Approaches to Quantum Gravity, ed. by D. Oriti, pp. 111-128 (2009). arXiv:0709.3851 [hep-th]

57. A. Codello, R. Percacci, Fixed points of higher derivative gravity. Phys. Rev. Lett. 97, 221301 (2006). doi:10.1103/PhysRevLett.97. 221301. arXiv:hep-th/0607128

58. A. Codello, R. Percacci, C. Rahmede, Investigating the ultraviolet properties of gravity with a Wilsonian renormalization group equation. Ann. Phys. 324, 414 (2009). doi:10.1016/j.aop.2008.08. 008. arXiv:0805.2909 [hep-th]

59. K. Groh, S. Rechenberger, F. Saueressig, O. Zanusso, Higher derivative gravity from the universal renormalization group machine. PoS EPS-HEP2011, 124 (2011). arXiv:1111.1743 [hepth]

60. N. Ohta, R. Percacci, Higher derivative gravity and asymptotic safety in diverse dimensions. Class. Quantum Gravity 31, 015024 (2014). doi:10.1088/0264-9381/31/1/015024. arXiv:1308.3398 [hep-th]

61. N. Ohta, R. Percacci, Ultraviolet fixed points in conformal gravity and general quadratic theories. Class. Quantum Gravity 33, 035001 (2016). doi:10.1088/0264-9381/33/3/035001. arXiv:1506.05526 [hep-th]

62. N. Ohta, R. Percacci, A.D. Pereira, Gauges and functional measures in quantum gravity II: higher derivative gravity. Eur. Phys. J. C 77(9), 611 (2017). arXiv:1610.07991 [hep-th]

63. M.R. Niedermaier, Gravitational fixed points from perturbation theory. Phys. Rev. Lett. 103, 101303 (2009). doi:10.1103/ PhysRevLett.103.101303

64. D. Benedetti, P.F. Machado, F. Saueressig, Asymptotic safety in higher-derivative gravity. Mod. Phys. Lett. A 24, 2233 (2009). doi:10.1142/S0217732309031521. arXiv:0901.2984 [hep-th]

65. D. Benedetti, P.F. Machado, F. Saueressig, Taming perturbative divergences in asymptotically safe gravity. Nucl. Phys. B 824, 168 (2010). doi:10.1016/j.nuclphysb.2009.08.023. arXiv:0902.4630 [hep-th]

66. L. Modesto, Super-renormalizable quantum gravity. Phys. Rev. D 86, 044005 (2012). doi:10.1103/PhysRevD.86.044005. arXiv:1107.2403 [hep-th]

67. T. Biswas, E. Gerwick, T. Koivisto, A. Mazumdar, Towards singularity and ghost free theories of gravity. Phys. Rev. Lett. 108, 031101 (2012). doi:10.1103/PhysRevLett.108.031101. arXiv: 1110.5249 [gr-qc]

68. L. Modesto, L. Rachwal, Super-renormalizable and finite gravitational theories. Nucl. Phys. B 889, 228 (2014). doi:10.1016/j. nuclphysb.2014.10.015. arXiv:1407.8036 [hep-th]

69. E.T. Tomboulis, Renormalization and unitarity in higher derivative and nonlocal gravity theories. Mod. Phys. Lett. A 30(03n04), 1540005 (2015). doi:10.1142/S0217732315400052

70. B.S. DeWitt, A Gauge invariant effective action, in Oxford 1980, Proceedings, Quantum Gravity 2, pp. 449-487 and Calif. Univ.
Santa Barbara-NSF-ITP-80-031 (80,REC.AUG.) 54 P. (009106) (see conference index)

71. L.F. Abbott, The background field method beyond one loop. Nucl. Phys. B 185, 189 (1981)

72. L.D. Faddeev, V.N. Popov, Feynman diagrams for the Yang-Mills field. Phys. Lett. B 25, 29 (1967)

73. R.E. Kallosh, Modified Feynman rules in supergravity. Nucl. Phys. B 141, 141 (1978)

74. N.K. Nielsen, Ghost counting in supergravity. Nucl. Phys. B 140, 499 (1978)

75. B.S. DeWitt, Dynamical theory of groups and fields. Conf. Proc. C 630701, 585 (1964) [Les Houches Lect. Notes 13, 585 (1964)]

76. D.V. Vassilevich, Heat kernel expansion: user's manual. Phys. Rep. 388, 279 (2003). doi:10.1016/j.physrep.2003.09.002. arXiv:hep-th/0306138

77. S. Yajima, Evaluation of heat kernel in curved space. Class. Quantum Gravity 5, L207 (1988). doi:10.1088/0264-9381/5/12/003

78. I.G. Avramidi, Heat kernel and quantum gravity. Lect. Notes Phys. Monogr. 64, 1 (2000). doi:10.1007/3-540-46523-5

79. J.M. Martín-García, xAct: efficient tensor computer algebra. http:// www.xact.es

80. T. Nutma, xTras: a field-theory inspired xAct package for mathematica. Comput. Phys. Commun. 185, 1719 (2014). arXiv: 1308.3493 [cs.SC]

81. R. Mertig, M. Bohm, A. Denner, FEYN CALC: computer algebraic calculation of Feynman amplitudes. Comput. Phys. Commun. 64, 345 (1991). doi:10.1016/0010-4655(91)90130-D

82. E. Elizalde, S.D. Odintsov, A. Romeo, Renormalization group properties of higher derivative quantum gravity with matter in (4epsilon)-dimensions. Nucl. Phys. B 462, 315 (1996). doi:10.1016/ 0550-3213(95)00674-5. arXiv:hep-th/9502131

83. E.V. Gorbar, I.L. Shapiro, Renormalization group and decoupling in curved space. JHEP 0302, 021 (2003). doi:10.1088/1126-6708/ 2003/02/021. arXiv:hep-ph/0210388

84. E.V. Gorbar, I.L. Shapiro, Renormalization group and decoupling in curved space. 2. The standard model and beyond. JHEP 0306, 004 (2003). doi:10.1088/1126-6708/2003/06/004. arXiv:hep-ph/0303124

85. E.V. Gorbar, I.L. Shapiro, Renormalization group and decoupling in curved space. 3 . The case of spontaneous symmetry breaking. JHEP 0402, 060 (2004). doi:10.1088/1126-6708/2004/02/ 060. arXiv:hep-ph/0311190

86. Y. Yoon, Y. Yoon, Asymptotic conformal invariance of SU(2) and standard models in curved space-time. Int. J. Mod. Phys. A 12, 2903 (1997). doi:10.1142/S0217751X97001602. arXiv:hep-th/9612001

87. D.J. Gross, M.J. Perry, L.G. Yaffe, Instability of flat space at finite temperature. Phys. Rev. D 25, 330 (1982). doi:10.1103/PhysRevD. 25.330

88. S. Bhattacharjee, P. Majumdar, Gravitational Coleman-Weinberg potential and its finite temperature counterpart. Nucl. Phys. B 885, 481 (2014). doi:10.1016/j.nuclphysb.2014.05.031. arXiv: 1210.0497 [hep-th]

89. S.C. Lee, P. van Nieuwenhuizen, Counting of states in higher derivative field theories. Phys. Rev. D 26, 934 (1982). doi:10.1103/ PhysRevD.26.934

90. R.J. Riegert, The particle content of linearized conformal gravity. Phys. Lett. A 105, 110 (1984). doi:10.1016/ 0375-9601(84)90648-0

91. V. Branchina, E. Messina, Stability, Higgs boson mass and new physics. Phys. Rev. Lett. 111, 241801 (2013). doi:10.1103/ PhysRevLett.111.241801. arXiv:1307.5193 [hep-ph]

92. M.B. Einhorn, F. Larsen, Interacting quantum field theory in de Sitter vacua. Phys. Rev. D 67, 024001 (2003). doi:10.1103/PhysRevD. 67.024001. arXiv:hep-th/0209159 
93. A.M. Polyakov, De Sitter space and eternity. Nucl. Phys. B 797, 199 (2008). doi:10.1016/j.nuclphysb.2008.01.002. arXiv:0709.2899 [hep-th]

94. D. Marolf, I.A. Morrison, M. Srednicki, Perturbative S-matrix for massive scalar fields in global de Sitter space. Class. Quantum Gravity 30, 155023 (2013). doi:10.1088/0264-9381/30/15/ 155023. arXiv:1209.6039 [hep-th]

95. G. Narain, Green's function of the vector fields on DeSitter background. arXiv:1408.6193 [gr-qc]

96. E.T. Akhmedov, Lecture notes on interacting quantum fields in de Sitter space. Int. J. Mod. Phys. D 23, 1430001 (2014). doi:10.1142/ S0218271814300018. arXiv:1309.2557 [hep-th]
97. M. Maggiore, M. Mancarella, Nonlocal gravity and dark energy. Phys. Rev. D 90(2), 023005 (2014). doi:10.1103/PhysRevD.90. 023005. arXiv:1402.0448 [hep-th]

98. M. Maggiore, Nonlocal infrared modifications of gravity. A review. Fundam. Theor. Phys. 187, 221-281 (2017). arXiv:1606.08784 [hep-th]

99. T. Appelquist, J. Carazzone, Infrared singularities and massive fields. Phys. Rev. D 11, 2856 (1975). doi:10.1103/PhysRevD.11. 2856 